\title{
Aerodynamic Optimization of Building Augmented Wind TURBINES
}

\author{
by \\ John Adam MacQuarrie \\ Bachelor Aerospace Engineering, Ryerson, 2008
}

\author{
A thesis \\ presented to Ryerson University \\ in partial fulfillment of the \\ requirements for the degree of \\ Master of Applied Science \\ in the Program of \\ Aerospace Engineering
}

Toronto, Ontario, Canada, 2011

(C)John Adam MacQuarrie 2011 

I hereby declare that I am the sole author of this thesis.

I authorize Ryerson University to lend this thesis to other institutions or individuals for the purpose of scholarly research.

I further authorize Ryerson University to reproduce this thesis by photocopying or by other means, in total or in part, at the request of other institutions or individuals for the purpose of scholarly research. 

Aerodynamic Optimization of Building Augmented Wind Turbines

Master of Applied Science 2011

John Adam MacQuarrie

Aerospace Engineering

Ryerson University

\begin{abstract}
The omnipresence of wind, low production cost and much advancement within the field, wind power provides a vast and promising renewable energy resource. With the current high prices of oil and pressure to reduce carbon emissions, wind energy has achieved great interest creating high demands for innovative wind technology. Additionally, producing energy at the door step of consumers, such as at consumer dwellings in urban areas, wind power provides a means of producing efficient and reliable energy.

The use of architectural structures to provide an augmentation source for the wind has been pursued by some ambitious architects but the effects of building augmentation are still uncertain. This study used computational fluid dynamic models to analyze varying building geometries and their effects on power augmentation. A porous region was used to model a wind turbine back pressure across the gap between the buildings. Results show augmentation increases power production one to two times compared to equivalent size free-standing wind turbines. Results also show that certain wind incidence angles provide the best augmentation indicating that building geometry is optimal when design takes into consideration winds regularity azimuth.
\end{abstract}





\section{Acknowledgements}

I would like to thank my advisor Dr. Paul Walsh for giving me the opportunity to work with him on this insightful thesis.

I would also like to extend my gratitude to Dr. Jasson Lassaline for his technical support needed for the completion on this thesis.

I would like to thank the Shared Hierarchical Academic Research Computing Network (SHARCNET, www.sharcnet.ca) and Compute/Calcul Canada for providing me with the facilities to perform this immense study.

Lastly, I would like to extend tremendous gratitude to my mentor and friend Alberto Bueron for his wealth of support, guidance and voice of reason throughout this thesis. 



\section{Dedication}

This thesis is dedicated to my close friends and family for giving me the support and encouragement needed in the pursuit of this degree. 



\section{Contents}

$\begin{array}{lll}1 & \text { Introduction } & 1\end{array}$

1.1 Ducted Wind Turbines . . . . . . . . . . . . . . . . . . . . . . . . . . . . . 4

1.2 Building Augmented Wind Turbines $\ldots \ldots \ldots \ldots \ldots$

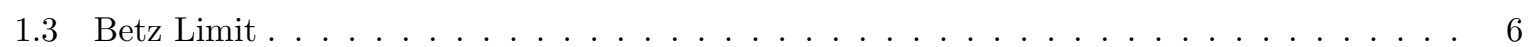

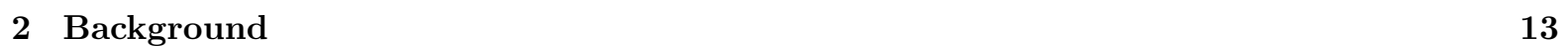

2.1 Flow Between Cylinder Pair . . . . . . . . . . . . . . . . . . . . . . . 13

2.2 Strouhal Number . . . . . . . . . . . . . . . . . . . . . . . . . . . . . . . . . . . . . . 14

2.3 Von Karman Street $\ldots \ldots \ldots \ldots$

2.4 2D Optimized BAWT Footprint $\ldots \ldots \ldots \ldots \ldots \ldots \ldots \ldots \ldots$

2.5 Statement of Purpose $\ldots \ldots \ldots \ldots \ldots \ldots \ldots \ldots$

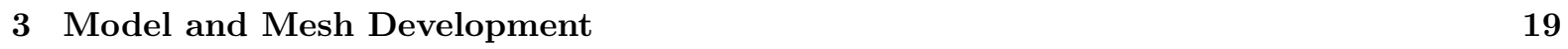

3.1 Modeling . . . . . . . . . . . . . . . . . . . . . . . . . . . . . . . . . . . . . . . . . 19

3.1 .1 Domain Boundaries $\ldots \ldots \ldots \ldots \ldots \ldots$

3.1 .2 Bodies of Influence . . . . . . . . . . . . . . . . . . . 24

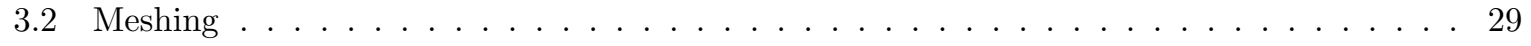

3.3 Inflation Layers $\ldots \ldots \ldots \ldots \ldots$

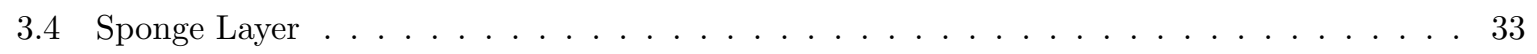

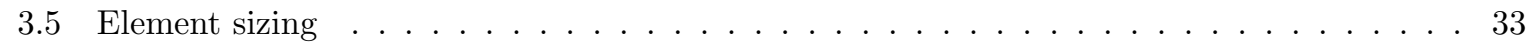

3.6 Grid and Time Step Study $\ldots \ldots \ldots \ldots \ldots$. . . . . . . . . . . . . . . . . . 34

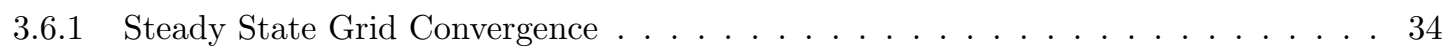

3.7 Time Step Study . . . . . . . . . . . . . . . . . . . . . . . . . . 38

3.8 Grid-Time Step Study $\ldots \ldots \ldots$. . . . . . . . . . . . . . . . . . . . . . . . . . . . . 39

\begin{tabular}{|lll}
\hline 4 & Methodology & 41
\end{tabular}

4.1 Boundary Conditions . . . . . . . . . . . . . . . . . . . . . . . 41

4.2 Solution Parameters $\ldots \ldots \ldots \ldots$. . . . . . . . . . . . . . . . . . . . . . . . 43

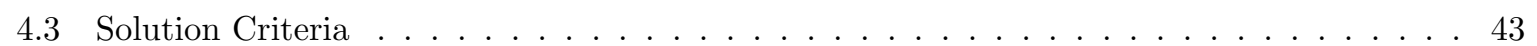

4.4 Augmented Power $\ldots \ldots \ldots \ldots \ldots$ 
$\begin{array}{llr}5 & \text { Results } & 49\end{array}$

5.1 Velocity Augmentation $\ldots \ldots \ldots \ldots \ldots$. . . . . . . . . . . . . . . . 49

5.2 Augmented Power $\ldots \ldots \ldots \ldots \ldots \ldots \ldots \ldots$

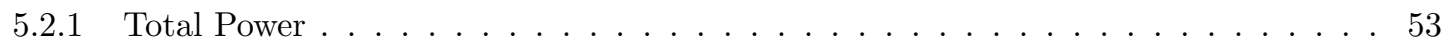

$5.2 .2 \quad$ Power Augmentation Factor $\ldots \ldots \ldots \ldots \ldots \ldots$

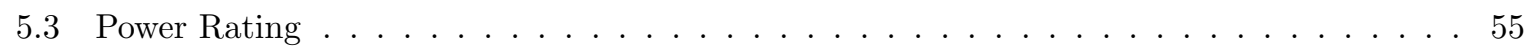

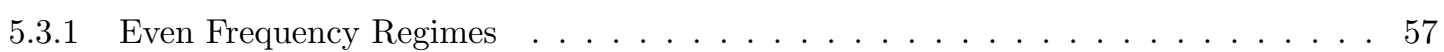

5.3 .2 Wind Regime Case Study . . . . . . . . . . . . . . . . . . . . . . . . 61

$\begin{array}{lll}6 & \text { Conclusion } & 65\end{array}$

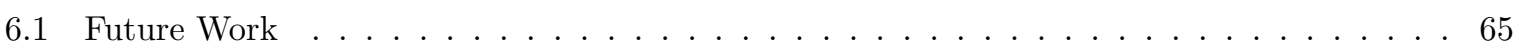

\begin{tabular}{ll}
\hline Bibliography & 106
\end{tabular} 


\section{List of Tables}

2.1 Strouhal numbers and frequencies in flow of Reynolds number $10^{6} \quad \ldots \ldots$. . . . . . . 14

2.2 Optimal parameters for different wind variations $\ldots \ldots \ldots \ldots \ldots \ldots$

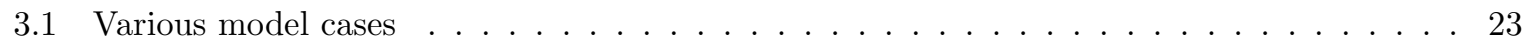

3.2 Element size controls . . . . . . . . . . . . . . . . . . . . . . . . . . . . 34

3.3 Steady state grid convergence and associated errors . . . . . . . . . . . . . . . . . . 38

3.4 Convergence of power with respect to time step size $\ldots \ldots \ldots \ldots \ldots$

3.5 Steady state grid convergence $\ldots \ldots \ldots \ldots \ldots \ldots$

$5.1 \quad$ Incidence angles and relative frequencies of wind regimes $\ldots \ldots \ldots \ldots$. . . . . . . 57

5.2 Wind regime of Toronto Island $\ldots \ldots \ldots \ldots \ldots \ldots \ldots$

5.3 Power rating for the wind regimes $\ldots \ldots \ldots \ldots \ldots \ldots$

B.1 Vortex propagation distances after three periods with respect to Strouhal numbers . . . . 69 



\section{List of Figures}

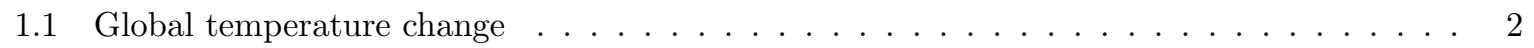

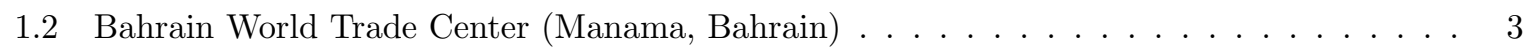

1.3 Schematic sketch of mean flow stream line pattern around a building . . . . . . . . . . . 4

1.4 Ducted turbines: Left: Roof top mounted, Right: Building integrated $\ldots . . . . . . .55$

1.5 Building integrated ducted turbine with spoiler $\ldots \ldots \ldots \ldots \ldots \ldots$

1.6 Two buildings used as concentrators $\ldots \ldots \ldots \ldots \ldots \ldots \ldots$

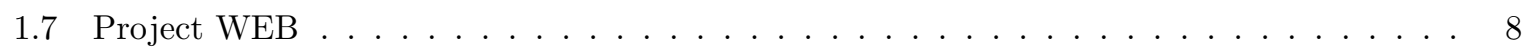

1.8 Project WEB performance $\ldots \ldots \ldots \ldots \ldots \ldots \ldots \ldots \ldots$

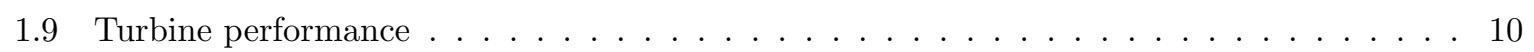

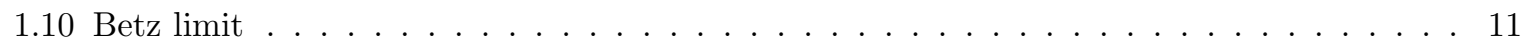

2.1 Vortex street from cylinder pair . . . . . . . . . . . . . . . . . . . . . . . 14

$2.2 \quad$ Iso-surface of Q-criterion depicting von Karman street and associated pressure $\ldots . . .15$

2.3 Oscillation of measured power for results of case $\left(R_{t}=0, R_{x}=0, R_{y}=0, \alpha=60^{\circ}\right)$. . 16

2.4 Varying parameters on a $2 \mathrm{D}$ building footprint $\ldots \ldots \ldots \ldots \ldots$

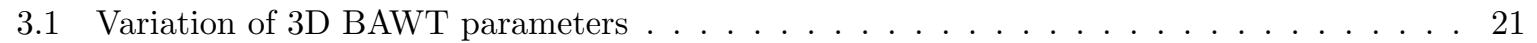

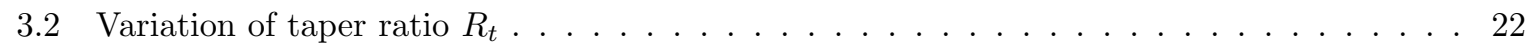

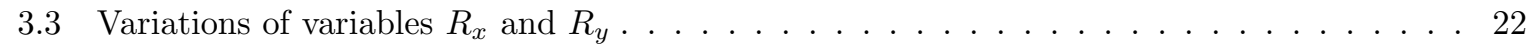

3.4 Front view of BAWT and simulated turbine capture area $\ldots \ldots \ldots \ldots \ldots$

3.5 Top view of turbine spans across and extends height of the BAWT $\ldots \ldots \ldots \ldots$

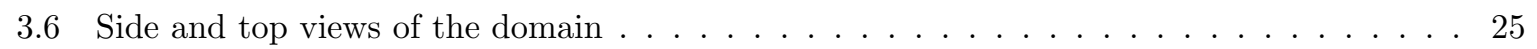

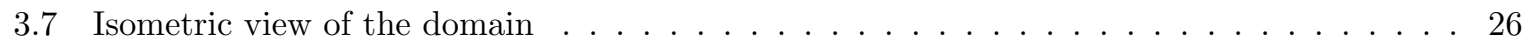

3.8 Close view of BAWT system with plain simulating turbines $\ldots \ldots \ldots \ldots$. . . . . 27

3.9 Bodies of influence defining the vortex street defined $\ldots \ldots \ldots \ldots \ldots$

3.10 Inflation layers against building facade $\ldots \ldots \ldots \ldots$

3.11 Unwrapped building surface's $y^{+}$values along building height $\ldots \ldots \ldots$. . . . . . . . . 32

3.12 Sponge layer defining the outlet boundary $\ldots \ldots \ldots \ldots \ldots \ldots \ldots$

3.13 Surface mesh of Walls, Turbine and Ground . . . . . . . . . . . . . . . . . . . . . 35

3.14 Mesh sliced lengthwise across Turbine . . . . . . . . . . . . . . . . . 36

3.15 Steady state mesh convergence $\ldots \ldots \ldots \ldots \ldots \ldots$ 


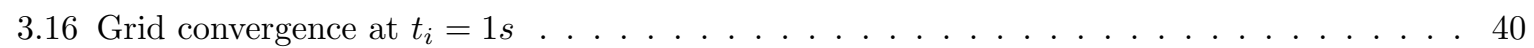

$4.1 \quad$ Log-Law velocity profile of ABL $\ldots \ldots \ldots \ldots \ldots$. . . . . . . . . . . . 42

$4.2 \quad$ Mean time power covergence study $\left(R_{t}=0.33, R_{x}=0, R_{y}=1, \alpha=0^{\circ}\right) \ldots \ldots \ldots 4$

$4.3 \quad$ Error covergence study $\left(R_{t}=0.33, R_{x}=0, R_{y}=1, \alpha=0^{\circ}\right) \ldots \ldots \ldots \ldots \ldots \ldots$

$4.4 \quad$ Mean time power covergence study $\left(R_{t}=0.33, R_{x}=0.5, R_{y}=1, \alpha=60^{\circ}\right) \ldots \ldots$

5.1 Results. . . . . . . . . . . . . . . . . . . . . . . 50

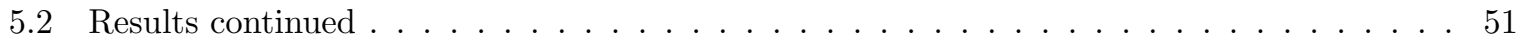

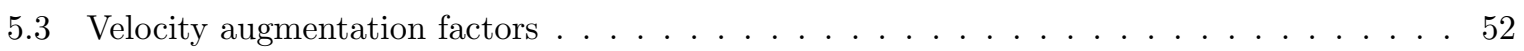

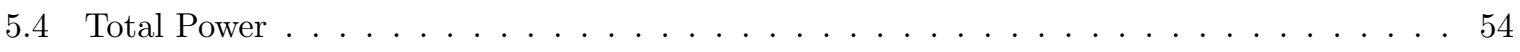

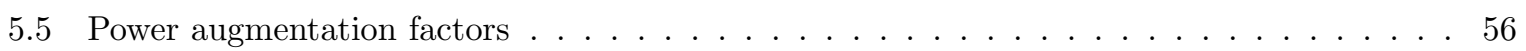

5.6 Power rating for high regime $\ldots \ldots \ldots \ldots \ldots \ldots \ldots \ldots$

5.7 Power rating for medium regime $\ldots \ldots \ldots \ldots \ldots \ldots$

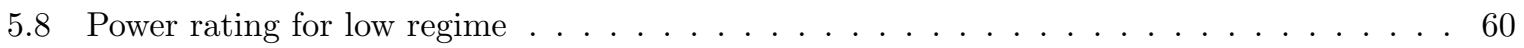

5.9 Toronto Island yearly average wind rose plot $\ldots \ldots \ldots \ldots \ldots \ldots$

5.10 BAWT azimuth on wind rose plot $\ldots \ldots \ldots \ldots \ldots \ldots$

5.11 Power rating for Toronto Island case study $\ldots \ldots \ldots \ldots \ldots$

B.1 Body of influence . . . . . . . . . . . . . . . . . . . . . . . . . . . 70

B.2 Q-Criterion Iso Surface with Turbulent kinetic energy variations $\left(R_{t}=0.33, R_{x}=0.5\right.$,

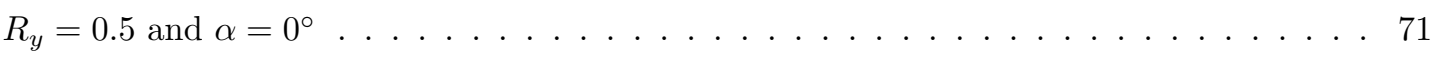

B.3 Q-Criterion Iso Surface with Turbulent kinetic energy variations $\left(R_{t}=0\right.$ and $\alpha=60^{\circ} \quad \ldots 72$

C.1 Pressure reading through BAWT a model $\ldots \ldots \ldots \ldots \ldots \ldots$

D.1 Contour of pressure on BAWT walls the pressure contour through the mid-plane projected on the domain side wall $\ldots \ldots \ldots \ldots \ldots \ldots \ldots \ldots$

D.2 Flow stream lines $\left(R_{t}=0, R_{x}=0, R_{y}=0\right.$ and $\left.\alpha=0\right) \ldots \ldots \ldots \ldots$ 


\section{List of Appendices}

\begin{tabular}{|l|l}
\hline A Parabolic BAWT Profile Formulation & 67
\end{tabular}

\begin{tabular}{|lr}
\hline B Bodies of Influence & 69
\end{tabular}

\begin{tabular}{|ll}
\hline C Porous Jump & 73 \\
\hline
\end{tabular}

\begin{tabular}{ll}
\hline D Figures & 77
\end{tabular}

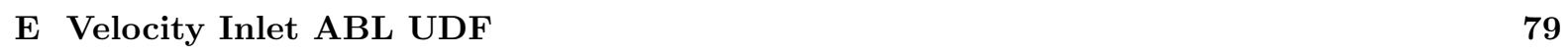

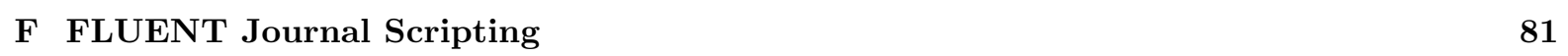

\begin{tabular}{|cc}
\hline G Shell Bash Script & 83 \\
\hline
\end{tabular}

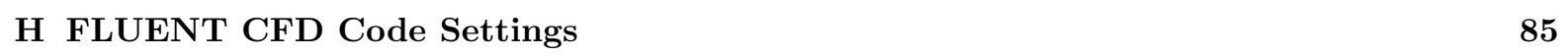

\begin{tabular}{|lr}
\hline I Sample Output File & 95
\end{tabular} 



\title{
List of Symbols
}

\author{
Re Reynolds number \\ $S_{t} \quad$ Strouhal number \\ $\Delta y \quad$ Distance of first element off the wall \\ $\alpha \quad$ Permeability of the porous media \\ $\mu \quad$ Fluid viscosity \\ $\tau \quad$ Shear stress \\ $n \quad$ Number of inflated prism layers \\ $y^{+} \quad$ Nondimensional distance \\ $l_{v p} \quad$ Vortex propagation distance \\ $N_{i} \quad$ Number of iterations \\ $P_{i} \quad$ Power at $\mathrm{i}^{\text {th }}$ iteration \\ $R_{t} \quad$ taper ratio \\ $R_{x} \quad$ forward lean \\ $R_{y} \quad$ building spread \\ $t \quad$ Time \\ $t_{i} \quad$ Time at $\mathrm{i}^{\text {th }}$ timestep \\ $u \quad$ Velocity in the $x$-direction \\ $u_{p} \quad$ Potential Velocity \\ $z \quad z$-displacement \\ $z_{o} \quad$ Surface roughness height
}




$\begin{array}{ll}\chi & \text { Placeholder } \\ \varnothing & \text { diameter } \\ C_{2} & \text { Coefficient of pressure } \\ \Delta m & \text { Porous jump thickness } \\ \Delta P & \text { Pressure drop } \\ \rho & \text { Density }\end{array}$




\section{Chapter 1}

\section{Introduction}

Wind power is generating interest amongst many nations to produce cheaper and sustainable electrical power to combat the soaring prices on fossil fuels and to reduce $\mathrm{CO}_{2}$ emissions [20. It is well known and documented that $\mathrm{CO}_{2}$ emissions from burning fossil fuels is contributing to global warming which has been felt all around the world. Figure 1.1 shows the average global temperature change documented by NASA indicating a dramatic rise in temperature caused by $\mathrm{CO}_{2}$ emissions [3]. Fox [6] stated the world's energy demand has increased two fold from 1971 to 2003, and currently will increase $40 \%$ by the year 2020. It is imperative that renewable energies is further developed and integrated into power systems to reduce fossil fuel emissions.

Compared to other renewable energies such as hydro, wave energy and solar, wind energy is the most promising form of renewable energy. It has sustained a $25 \%$ compounded rate of growth for over a decade and has exceeded a total capacity of $60,000 \mathrm{MW}$ [6]. The winds ability to produce power any hour of the day and season makes it a good source of energy [6]. Vast wind farms far from city centers have been constructed to mitigate the pressure of increased power demands due to growing urban areas. However, the great lengths of transmission lines used in power delivery to the areas are expensive to build and maintain, which makes wind farm energy less of an economical energy resource.

The problem of the integration of wind turbines into the urban environment is the spatial constraints imposed on the turbine size. Wind turbines extract kinetic energy from the wind through the turbine capture area and power increases capture area. Imposing size limit constrains turbines to produce little energy and making wind energy in urban environments not appealing.

A way to combat the size constraints was to increase power by increasing kinetic energy of the air. One method was to accelerate air velocities through a duct and a turbine by means of a pressure drop. It was shown ducted turbines boosted power and became integrated into the roof tops of some commercial building.

Research has been done by Campbell [22] to utilize the airflow between buildings where wind speeds increase to pass through narrow gaps. The increased wind velocity that occurs between building struc-

tures is known as the venturi effect which many researchers are utilizing to increase kinetic energy of the wind to drive smaller turbines to produce equivalent energy yields to larger turbines in open fields. 


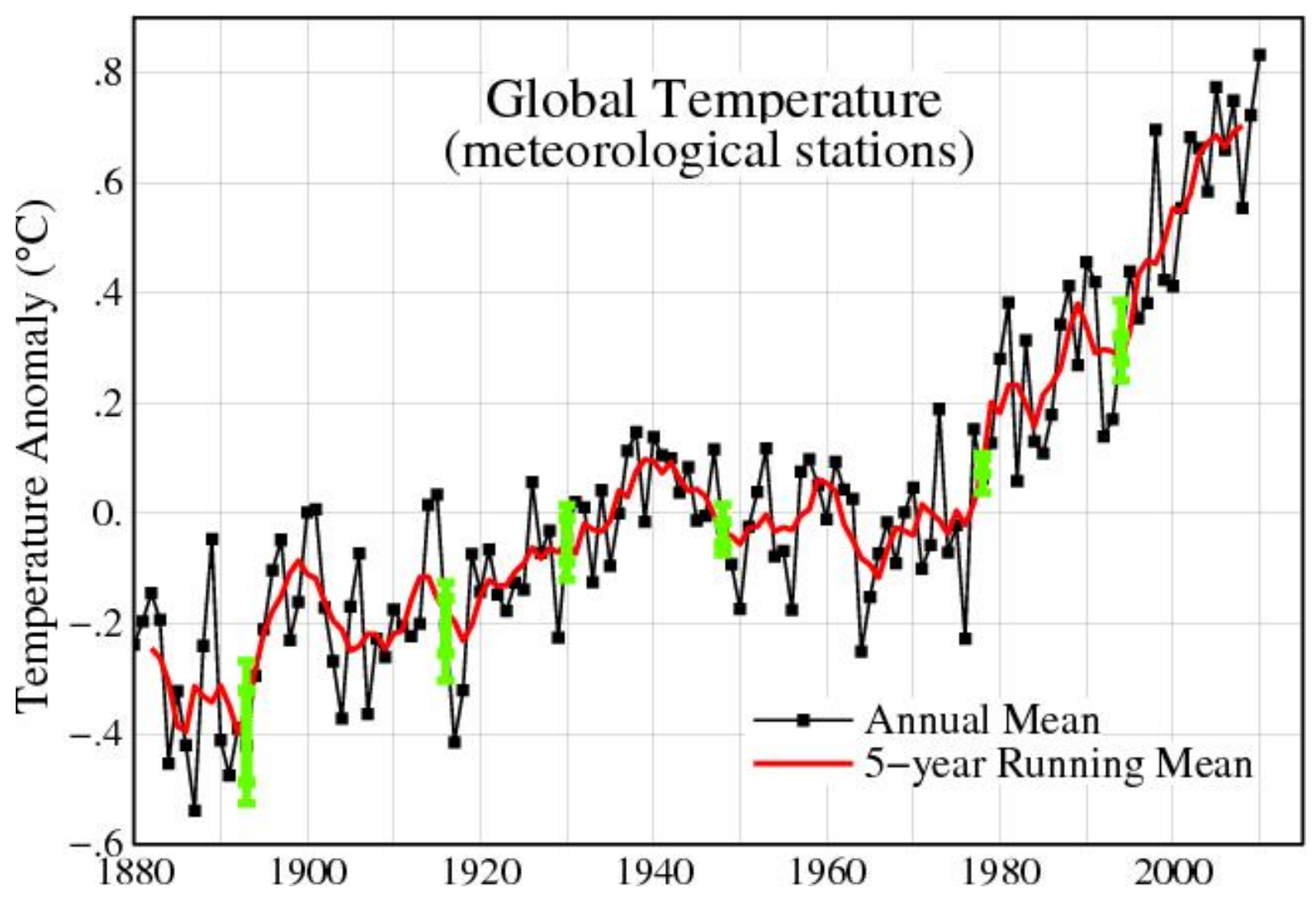

Figure 1.1: Global temperature change

[3] 
The size of urban turbines are constrained by space in which makes them less viable compared with their larger stand alone counterparts 22]. Concerns with the implementation of turbine into urban areas are the energy yield and the return on investment.

A system where two buildings are used to increase the winds kinetic energy is called building augmented wind turbine (BAWT). The Bahrain World Trade Center (BWTC) shown in Figure 1.2, was the first BAWT system to have been erected in 2008. Three $29 \mathrm{~m}$ diameter turbines are suspended between its two tower facing north to take advantage of the blowing winds from the Persian Gulf from a $90^{\circ}$ azimuth. The BWTC was shown to produce $11 \%-15 \%$ of the buildings electrical power [22]. Reasonings for the architectual building shape and optimized performance test results were not obtained.

Campbell [22] found in his research an optimal shape that would generate best power augmentation. He derived his shape using computational fluid dynamics (CFD) to analyse the shapes of the buildings and verified his results with scaled wind tunnel and field tests.

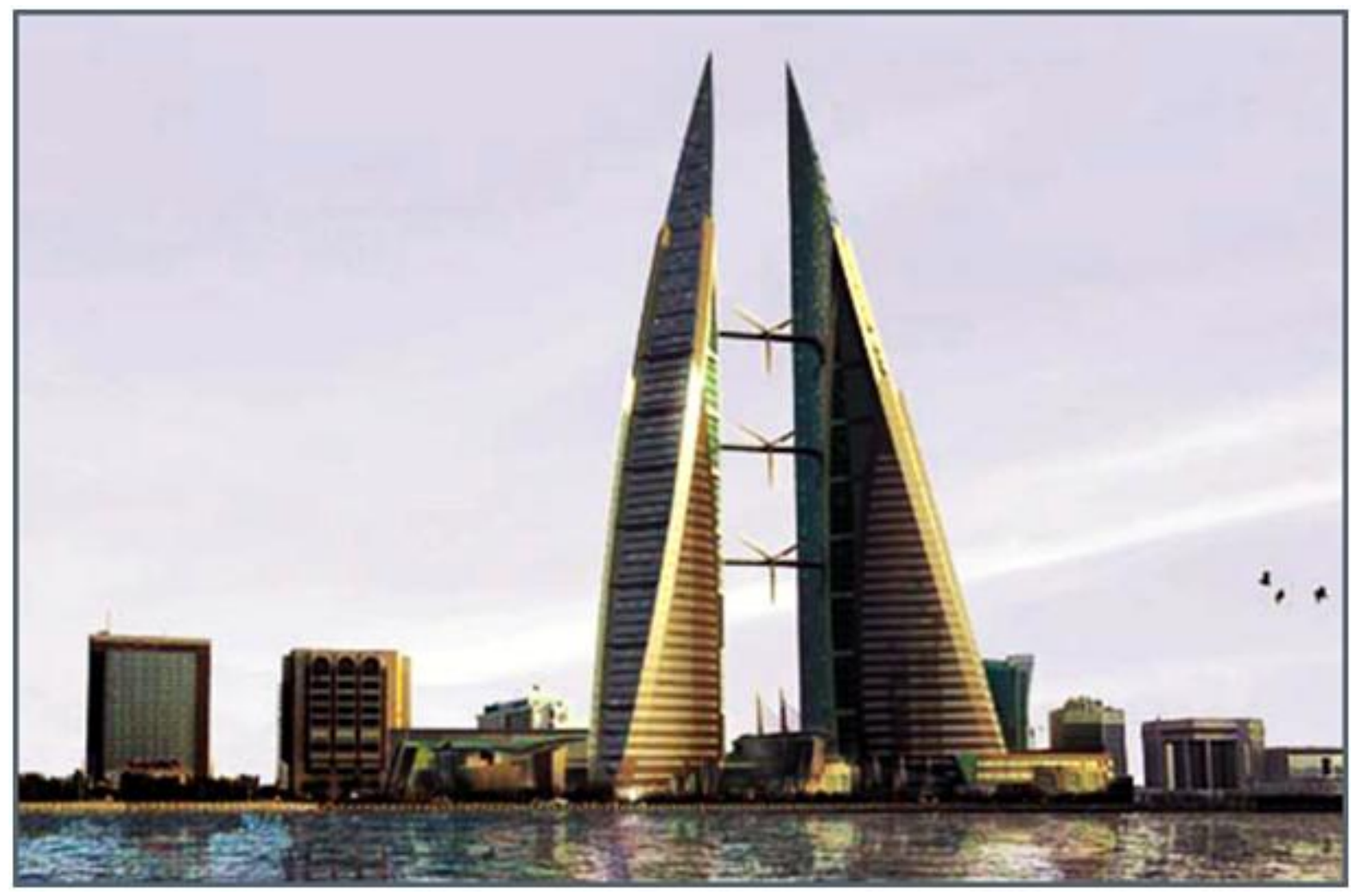

Figure 1.2: Bahrain World Trade Center (Manama, Bahrain)

This study uses CFD (computational fluid dynamics) software to compute the mass flow of air driven through a series of BAWT configurations with a simplified turbine model and compares the total power captured by the various configurations. This thesis will show that building shapes can be varied for maximizing power generation by wind augmentation.

Wind turbines are only $60 \%$ effective which is called the Betz limit and will be derived in subsequent 
sections. It should be noted that some published studies made the mistake to state that the augmentation increases performance past Betz limit. An augmentation of the wind has only taken place to accelerate velocities through the rotor where the turbine can extract a maximum power factor of $59.3 \%$.

\subsection{Ducted Wind Turbines}

Ducted wind turbines are categorized as small wind turbines which and are situated inside or at the end of air duct. These turbines are mounted generally on a roof top or integrated into the buildings facade. One end receives ambient air to be accelerated through the duct and directed to the turbine and the exit flow is directed perpendicular to the outside wind. This is done to utilize the separated flow from the leading edge of the turbine unit or the buildings roof. Such setup produces a low pressure region which increases the mass flow and energy potential in the air. A schematic of the flow streamline passing over a building is shown in Figure 1.3 . Figure 1.4 is the first model of a ducted wind turbine produced in 1979 by a Glaswegian engineer [9].

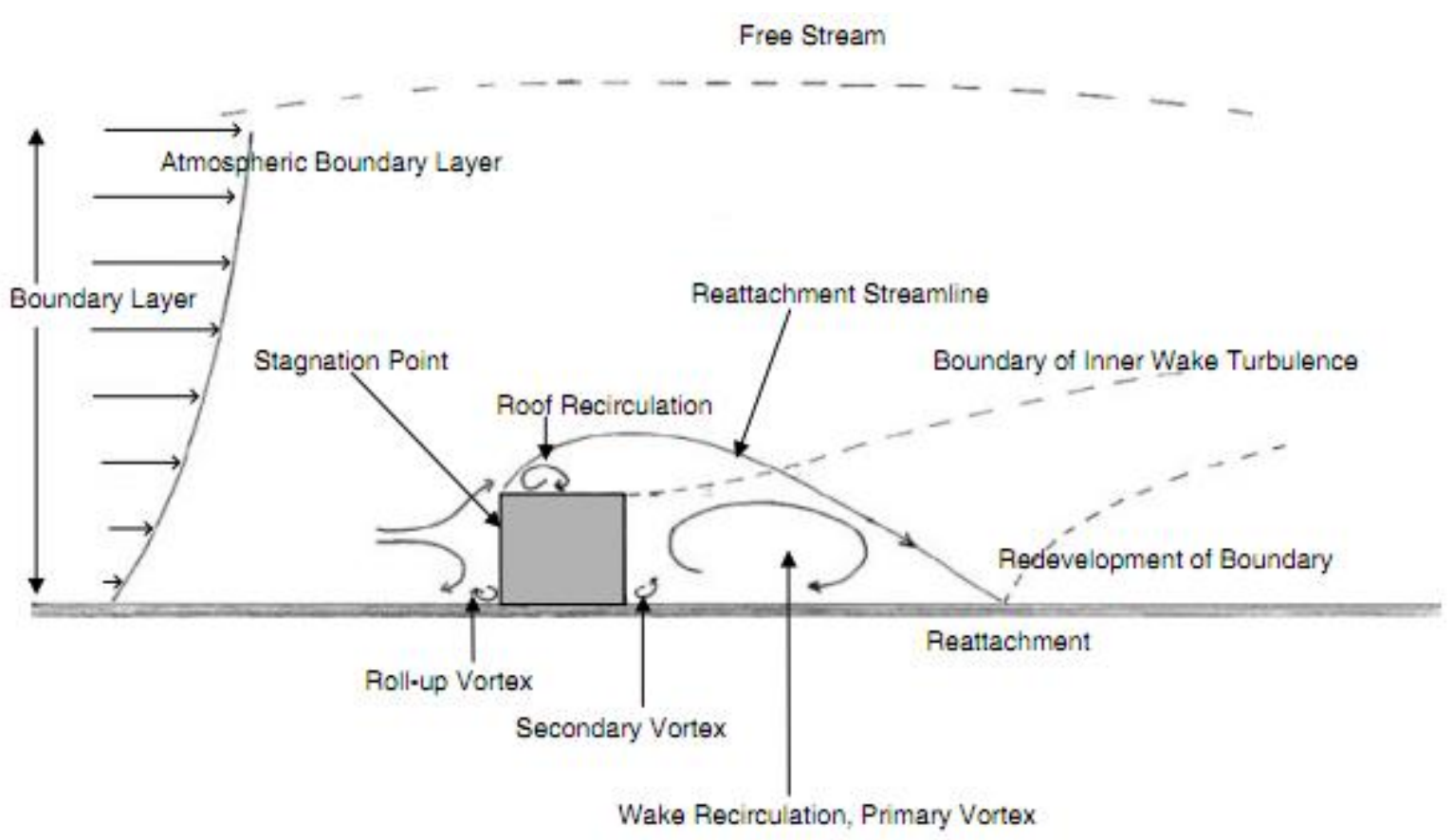

Figure 1.3: Schematic sketch of mean flow stream line pattern around a building

9]

Building integrated ducts increase air velocity from outside ambient air to the turbine by $20 \%$ and provide a 1.7 power increase compared to a bare stand-alone turbine of equal size. Spoilers can also be added to the top of the inlet to increase the performance. Spoilers slightly increase velocities from $30 \%$ to $40 \%$ at wind incidence angles up to $40^{\circ}$ with a power increase to 2.2 to 2.7 [9. Figure 1.5 shows a 

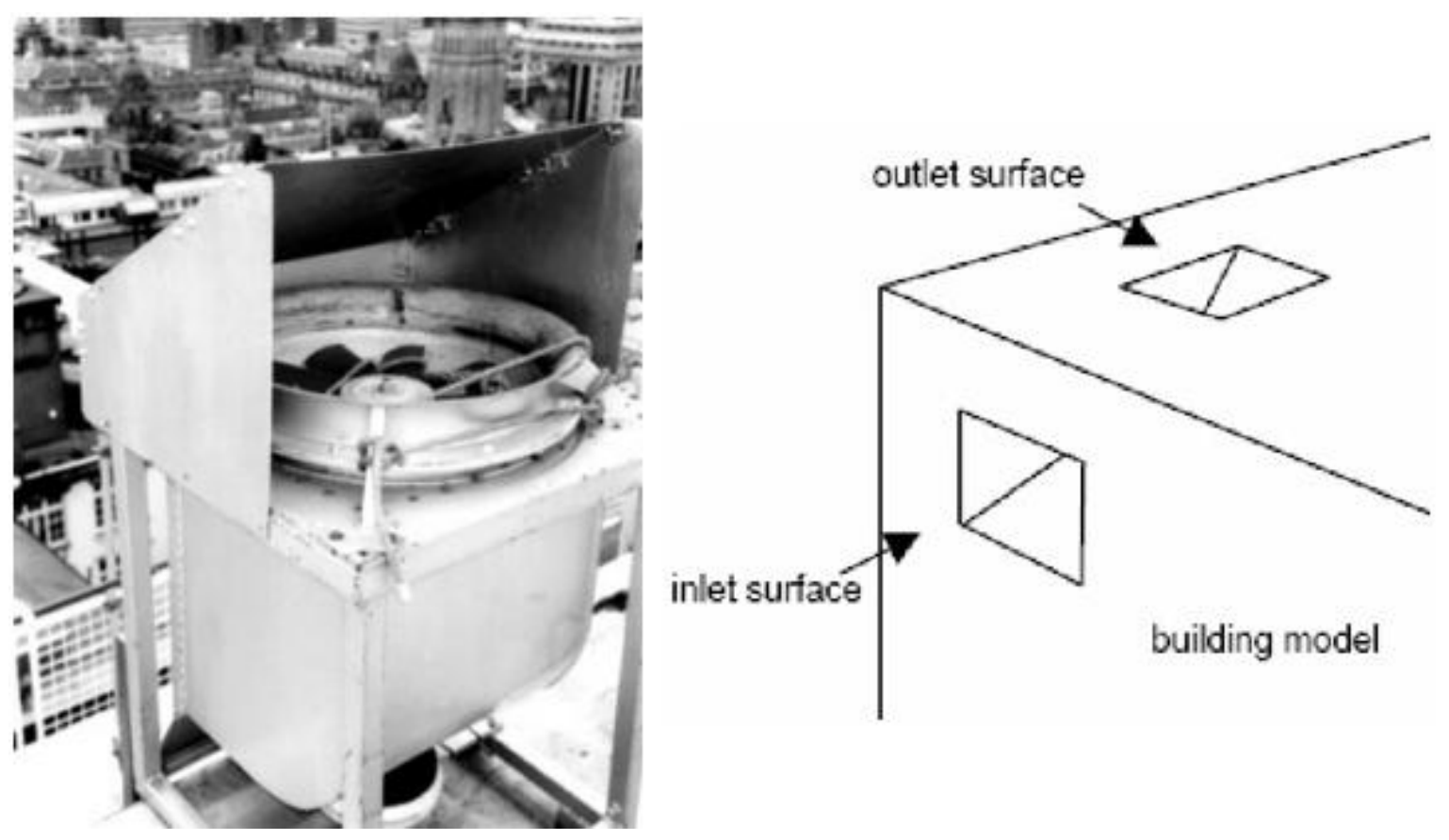

Figure 1.4: Ducted turbines: Left: Roof top mounted, Right: Building integrated 9]

spoiler mounted above the inlet of a building integrated wind turbine. Due to the size of ducted wind turbines, they are considered a small scale energy producer ranging from $250 \mathrm{~W}-500 \mathrm{~W}$.

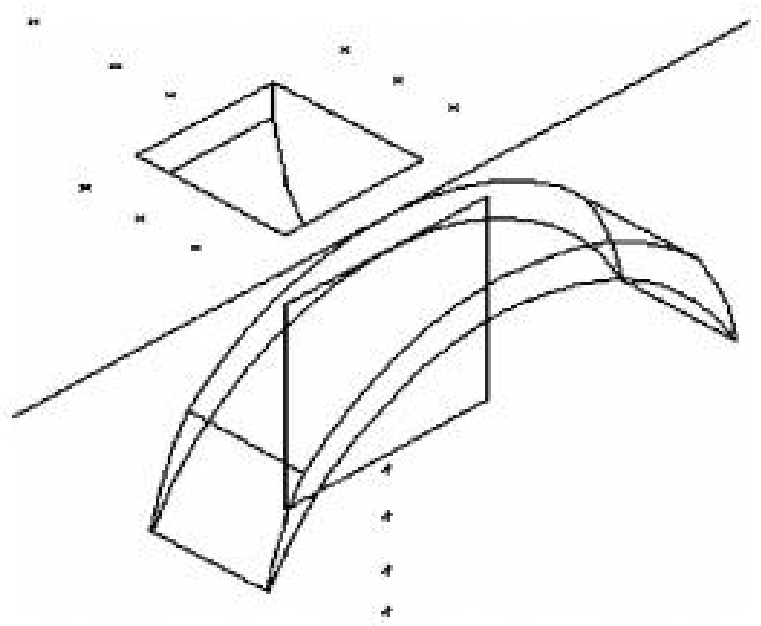

Figure 1.5: Building integrated ducted turbine with spoiler [9] 


\subsection{Building Augmented Wind Turbines}

A building augmented wind turbine (BAWT) system utilizes the venturi effect where the facade of the building is used to create a pressure drop through the system to increase the kinetic energy of the wind at the turbine rotor. Augmentation of the wind using buildings can be done either by integrating and retro-fitting existing buildings with wind turbines or designing buildings aerodynamically.

There are different types of BAWT system that are in use today where turbines are mounted on the side or top of a building. This study is focused on BAWT systems where two buildings are acting as a wind augmenter with turbines suspended between the buildings. This type of BAWT is shown in Figure 1.6 .

Several studies have been performed in the exploration of two-building BAWT systems. Imperial College [5] developed and tested BAWT systems where the building designs were contrived by the aerodynamic performance of the augmenters. This project is known as Wind Energy for the Built Environment (Project WEB) 22] and is shown in Figure 1.7. The key findings from that research were that optimal augmenter geometries were smooth, rounded and resembled a structural footprint of a 'kidney' or 'boomerang' shape. It was also found that losses due to flow separation could be reduced by incorporating closely fitted wings or an infill to shroud the turbine and good wind augmentation could be obtained at high angles of incidences up to $75^{\circ}[5]$.

Results from the Project WEB are shown in Figure 1.8. Substantial power enhancement up to 2.5 was obtained for low velocities and considerable power enhancement around 1.5 was obtained for speeds greater than $5 \mathrm{~m} / \mathrm{s}$. Figure 1.8 also shows good power enhancements at $0^{\circ}, 30^{\circ}$, and $60^{\circ}$ incidence and power generation still occurs at a wind incidence angle of $90^{\circ}$.

There were some issues raised by Campbell [22] concerning the environmental integration of the BAWT systems. Aerodynamically optimal designs may prove suboptimal economically and the buildings performance may not be energy efficient. Floor plans may not provide practical living space for the occupying residence. Spaces adjacent to the turbines would be less inviting due to concerns over noise, flickering of rotating blades and electromagnetic interference with electronic devices. Campbell suggested the possible solutions to tackle these concerns by placing service areas such as elevators and stairs adjacent to the turbines where these service areas could act as a buffer to the occupied spaces [22].

\subsection{Betz Limit}

The principle of wind turbines is to extract kinetic energy from the prevailing wind and convert it into electricity. A limit to the maximum extractable kinetic energy from the wind by a wind turbine was theorized to be $59.3 \%$ by Betz in 1919 [13], and is appropriately termed the Betz limit. Betz formulated the limit using simple one dimensional Bernoulli equation to model incompressible wind flow across the rotor. The assumptions used in the derivation were a constant, uniformly distributed velocity and pressure profile across the turbine's seeping area. Due to this model is one dimensional, tip losses due to rotational effects and viscous dissipation are neglected. 


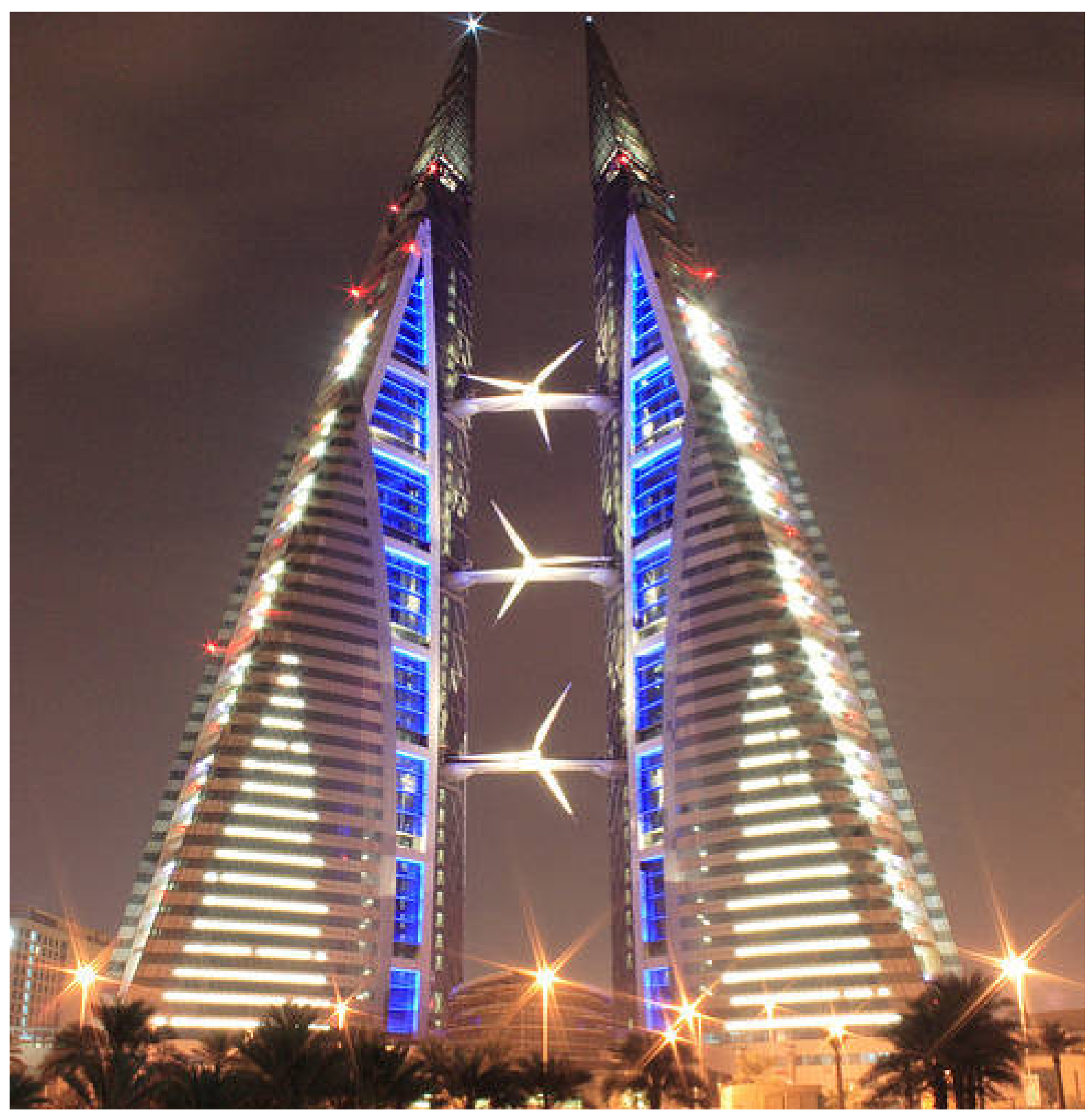

Figure 1.6: Two buildings used as concentrators 


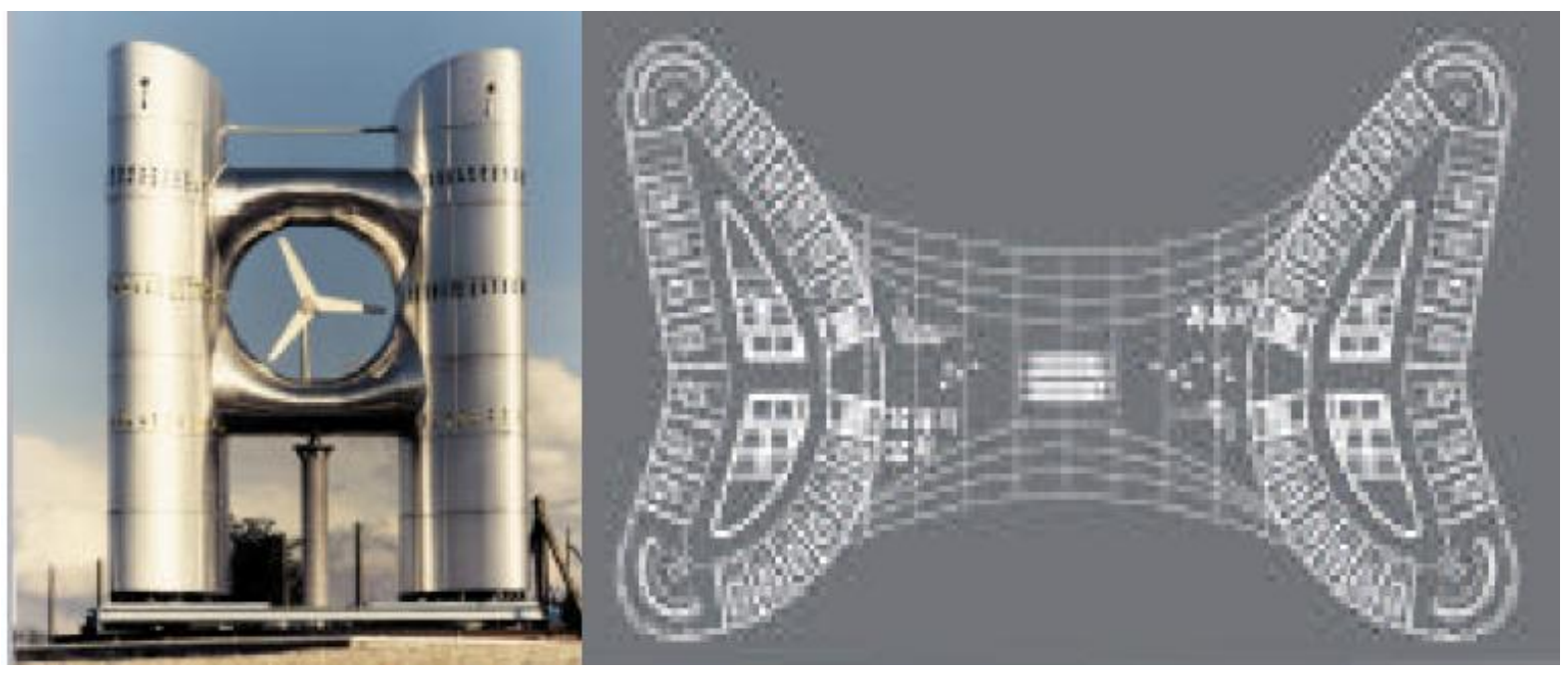

Figure 1.7: Project WEB

22

Referring to Figure 1.9, Betz assumed a stream tube of constant mass flow starting with the undisturbed wind flow defined as $V_{1}$, and $V_{2}$ as the velocity of the wind before and aft of the rotor. Also it was assumed the wind velocity at the turbines rotor is the mean velocity of the wind before and aft of the rotor. Thus the mean wind speed at the rotor is given as

$$
V=\frac{1}{2}\left(V_{1}+V_{2}\right)
$$

The assumption of incompressibility makes the mass flow of the given stream tube to be constant at the rotor inlet and is defined as

$$
\dot{m}=\rho A_{r} V
$$

where, $\rho$ is density of air, $A_{r}$ is the rotor sweeping area, $\dot{m}$ is the mass flow through turbine rotor area and $V$ is the mean velocity of air at the rotor. The kinetic energy extracted by the turbine is defined by the change in kinetic energy of the inlet and exit of the stream tube.

The Betz limit is defined as the ratio of change in kinetic energy to the undisturbed kinetic energy. Simplified, the power ratio is defined as

$$
P R=\frac{P}{P_{o}}=\frac{1}{2}\left(1-V R^{2}\right)(1+V R)
$$

where $V R=V_{2} / V_{1}$ is considered the velocity ratio. A graphical representation of Equation 1.3 is presented in Figure 1.10. The inflection point is the Bezt limit and is found by the minimization of the 


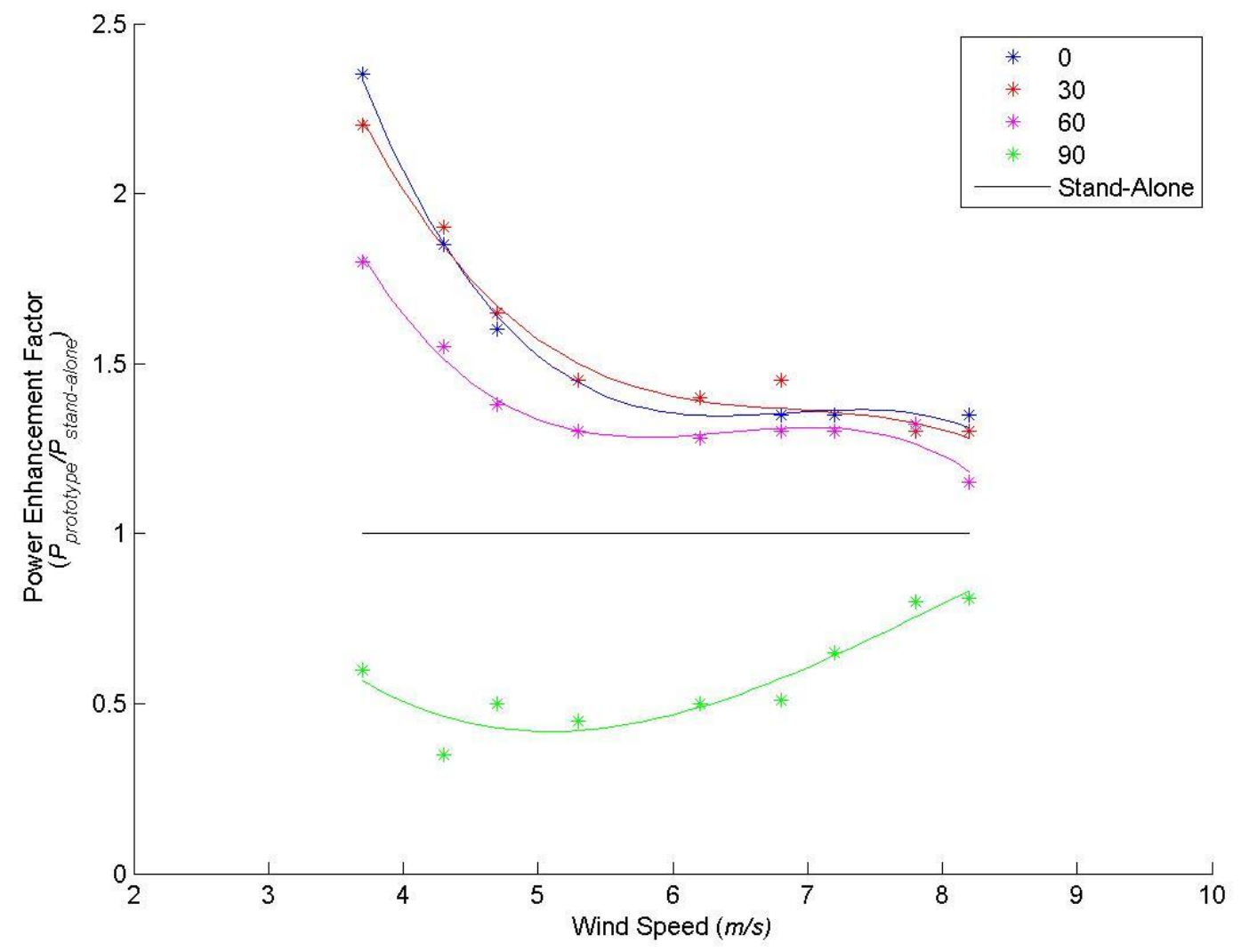

Figure 1.8: Project WEB performance

22 


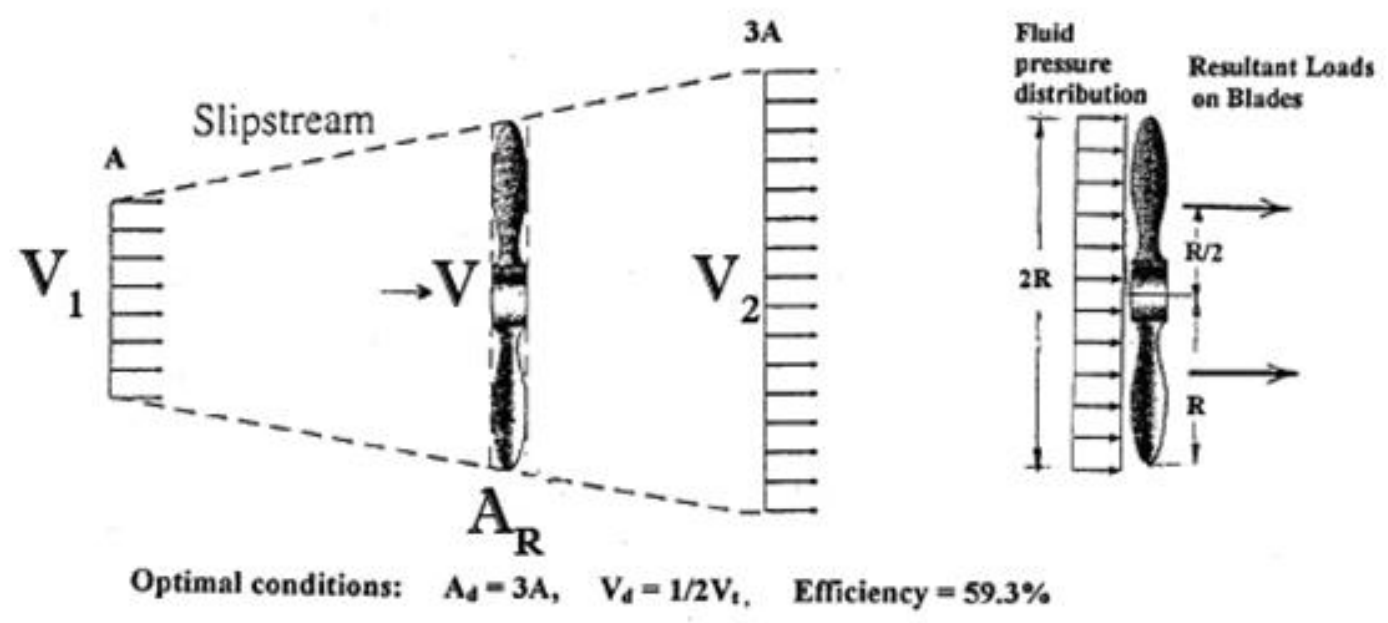

Figure 1.9: Turbine performance

13

differential form of Equation 1.3 to yield the velocity ratio corresponding to the maximum power ratio. Maximum power ratio of 0.593 is the Bezt limit and is represented in Figure 1.10 .

In reality the Betz limit cannot be achieved with a wind turbine alone due to aerodynamic losses, viscous dissipation, and efficiency losses though mechanical and electrical power converting devices. Such losses arise due to mechanical efficiency losses which reduces the power to $35 \%-45 \%$. There are energy converters, transmitters, generators and storage devices, the energy extracted drops to $10 \%-30 \%$ of the available potential wind power. However, engineers are using shrouded and ducted turbines to increase the overall mass flow to the turbines increasing the flows kinetic energy. These devices are known as wind augmentation devices because they increase the mass flow and kinetic energy at the rotor plane by utilizing the pressure differential to increase the energy density of the flow. 


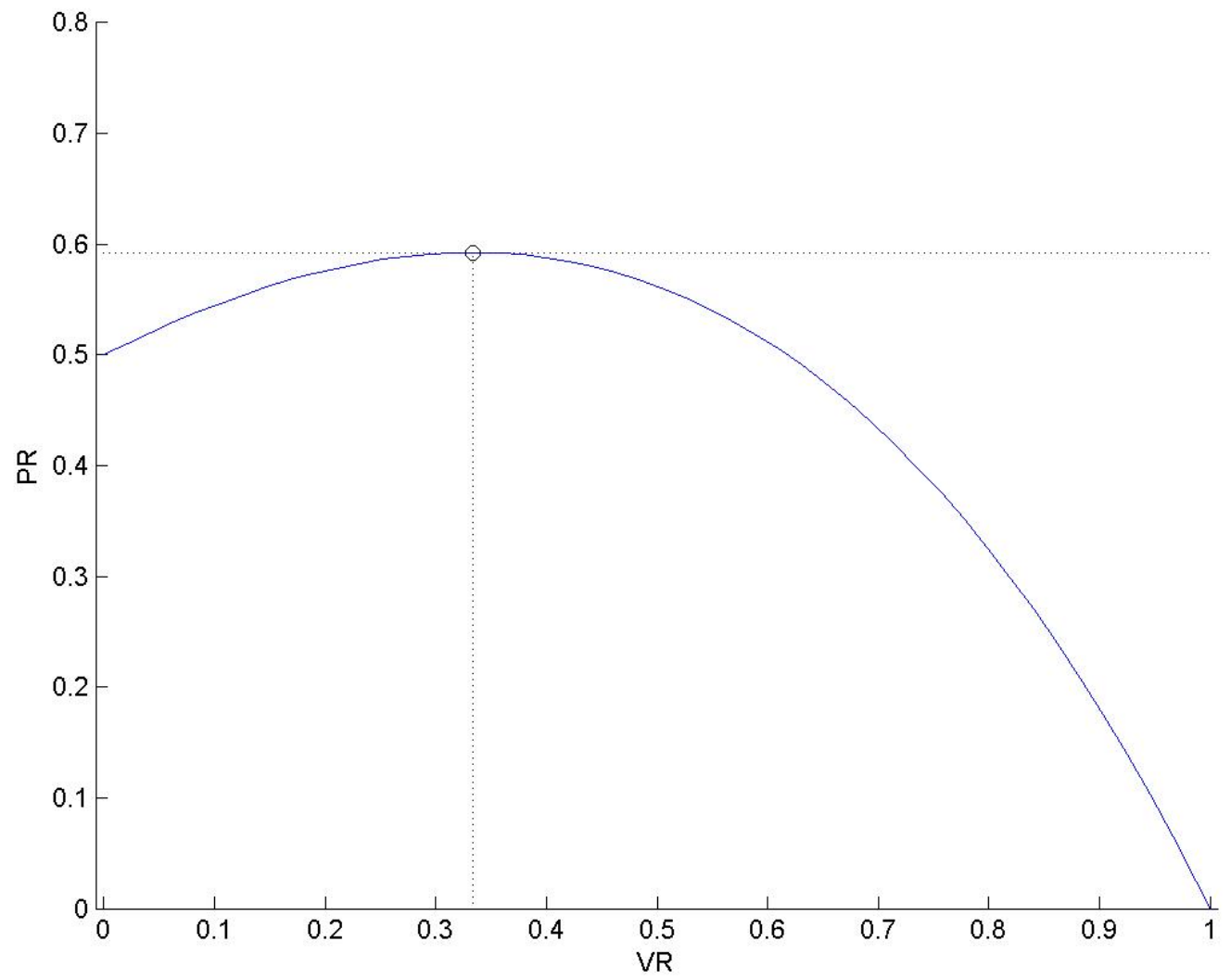

Figure 1.10: Betz limit 



\section{Chapter 2}

\section{Background}

It is well known that turbulence generated aft of an obstacle can affect the upstream flow characteristics and therefore proper modeling techniques of the turbulence aft of the BAWT systems were utilized. Vortex shedding occurs in a fluid as it passes blunt objects. Alternating low-pressure regions form behind objects which curls the flow into these regions creating a vortex. The vortex low-pressure regions propagate downstream and affect the pressure throughout the solution domain which is known as a pressure cascade. Modeling this pressure casade from vortex shedding will influence the flow characteristics upstream of the BAWT which affects the velocity of the flow passing through the Turbine boundary. This chapter presents the background information for the formulation of methods used to mode vortex shedding in the subsequent sections.

\subsection{Flow Between Cylinder Pair}

Investigation into flow around paired cylinders had been studied by many researchers like Bearman and Wadcock [7, and Kim and Durbin [17. Figure 2.1 shows the von Karman street aft of cylinder pairs in low flow Reynolds numbers.

The spatial distance separating the cylinder normalized by the cylinder diameter (gap ratio) was the subject of study. They concluded that with gap ratios of 0.5 to 1 , the flow is deflected from one body and back to the other in an alternating manner and becomes asymmetric downstream. For gap ratios less than 0.5, the flow around the two cylinders resembled a flow around a single bluff body and produced a single vortex street. The studies also noted that there were multiple modes and Strouhal Numbers for the quasi-stable and alternating behaviours complicating the determination of the vortex shedding period [7, 17]. 

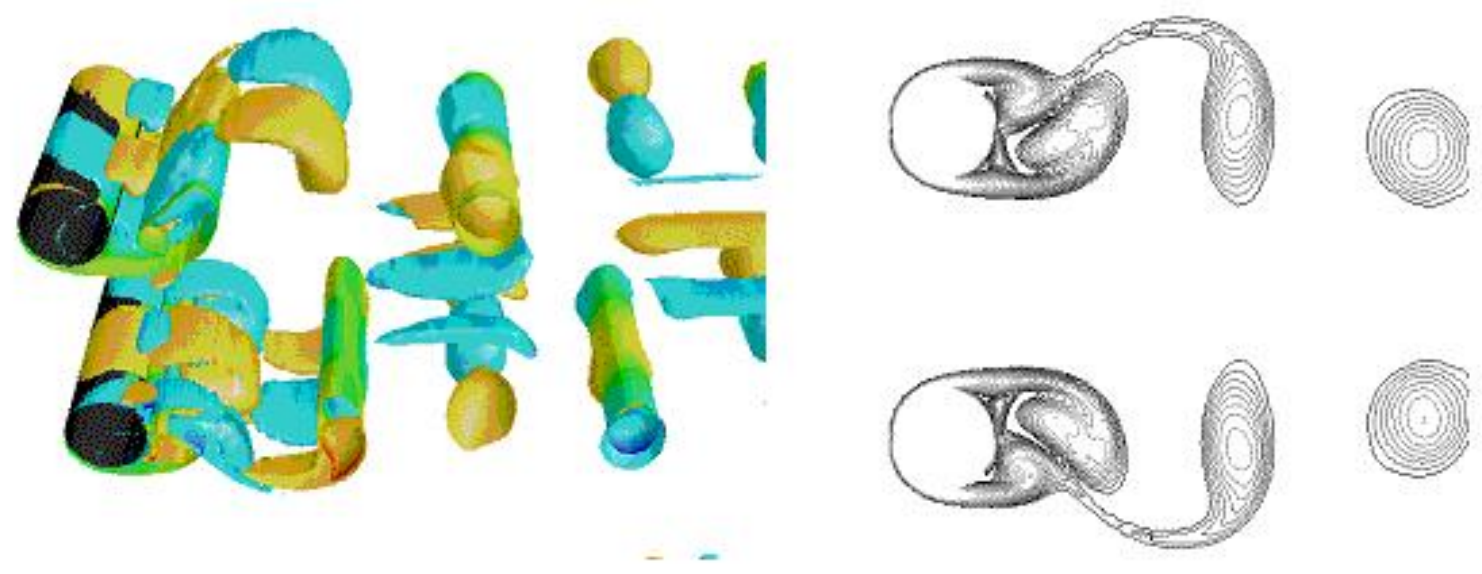

Figure 2.1: Vortex street from cylinder pair

8

\begin{tabular}{c|c}
$\begin{array}{c}\text { Strouhal } \\
\text { Numbers }\end{array}$ & $\begin{array}{c}\text { Frequency } \\
{[\mathrm{Hz}]}\end{array}$ \\
\hline 0.18 & 0.0374 \\
0.50 & 0.1040
\end{tabular}

Table 2.1: Strouhal numbers and frequencies in flow of Reynolds number $10^{6}$

24

\subsection{Strouhal Number}

The von Karman street which forms aft of the BAWT will develop regular periodic oscillation of vortex shedding. Physicist Strouhal described these oscillating frequencies of the vortex street from a circular cylinder as a dimensionless number appropriately named the Strouhal number. The formula for the Strouhal number is shown in Equation 2.1

$$
S_{t}=\frac{f_{r} L}{V}
$$

where $f_{r}$ is the frequency of the vortex shedding, $L$ is the characteristic length of the circular cylinders and $V$ is the velocity of the fluid. For Strouhal numbers high as 1 represent flows dominated by viscosity where slow oscillating motion happens. For Strouhal numbers to the order of $10^{-4}$, high speeds dominates the flow and intermediate number characterise turbulent flow with build up of shedding vortices [23]. Experimental results from Zdravkovich [24] on for flow over a cylinder at a Reynolds number of $10^{6}$ give Strouhal numbers ranging from $0.18-0.50$ and relative frequencies are given in Tabletab::Strouhal numbers.

For the purpose of this study a Strouhal number of 0.18 is used to predict the vortex shedding 
frequency using $L=20 \mathrm{~m}$ and $V=4.16 \mathrm{~m} / \mathrm{s}$ giving a frequency of $0.0374 \mathrm{~Hz}$ or and a shed period of $26.7 \mathrm{~s}$. Flow simulation accuracy can be achieved by using low order time steps to analyse the vortex structures. Due to the computational resources needed, CFD simulations were performed using 1s time step, one order less than the shedding period.

\subsection{Von Karman Street}

This study is focused on the augmented power ratio resultant of the BAWT geometry and transient pressure fluctuations created by the resultant von Karman street. The technique used in viewing vortices was given by [2] wherein creating an isoplot of the Q-Criterion at the lowest calculated value. The Qcriterion is a factor used in determining vortex structures in turbulence where flow rotation dominates strain [18. Using the ANSYS post processing program, an isoplot of the Q-Criterion at the lowest calculated value is created to describe the von Karman street generated downstream as shown in Figure 2.2 ,

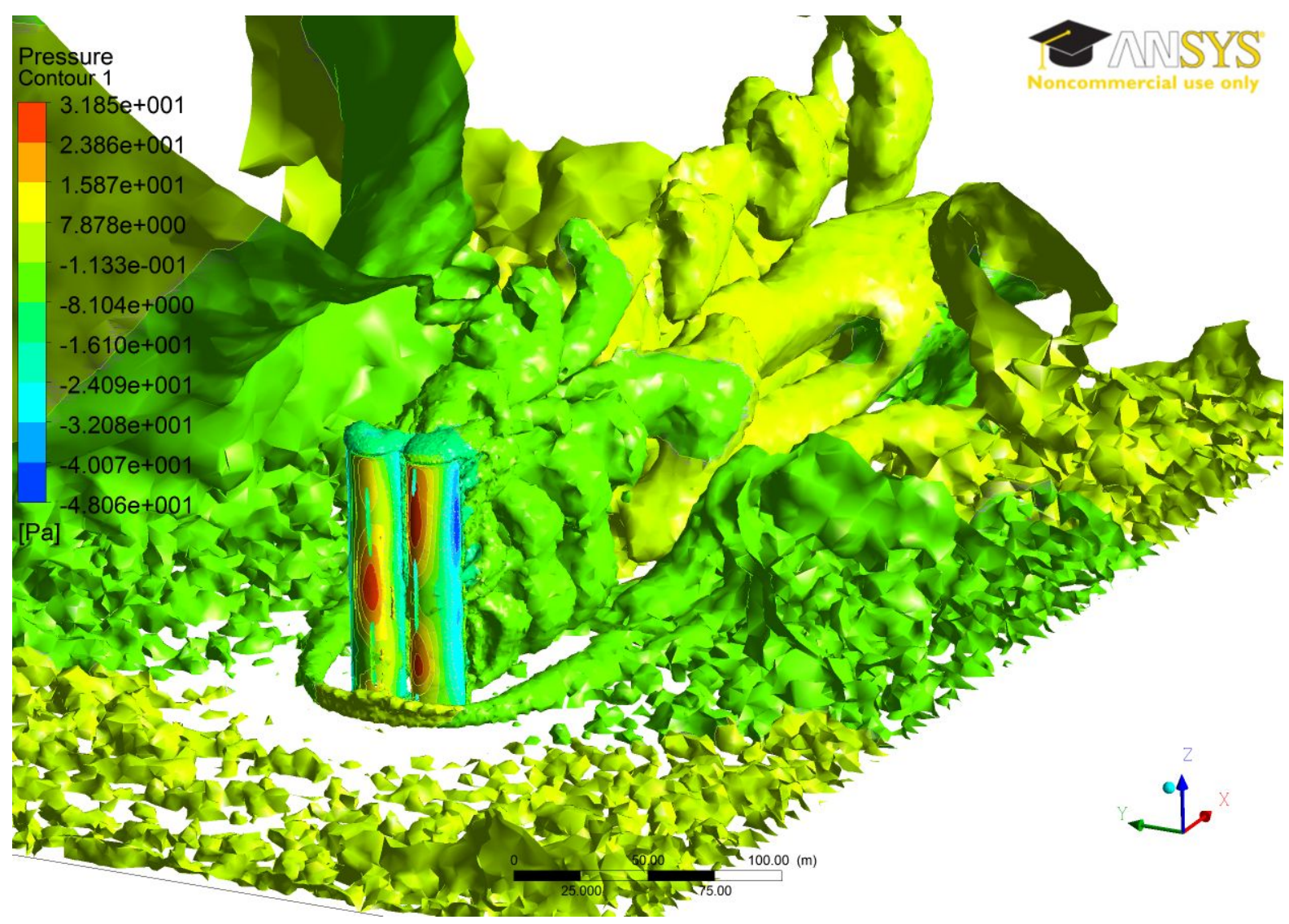

Figure 2.2: Iso-surface of Q-criterion depicting von Karman street and associated pressure

The technique used describes the von Karman street quite well and conforms to the size and frequency 
of the shed vortices previously mentioned in Table 2.1. It is shown in Figure 2.2 a periodic von Karman street forms downstream. The bubble formations are vortex rollups which occur after its been shed from the building. There are three vortecies within the designated vortex street clearly defined by the bodies of influence. Von Karman street conforms to the belief that the vortex size does not have considerable change downstream and falls within the expected Strouhal numbers.

Fluctuations of the measured augmented power were either small or insignificant at low wind incidence angles. Where at wind incidence angles of $40^{\circ}$ and $60^{\circ}$, oscillations in the measured augmented power are evident. Figure 2.3 is a look at a solution oscillating with respect at $60^{\circ}$ wind incidence angle.

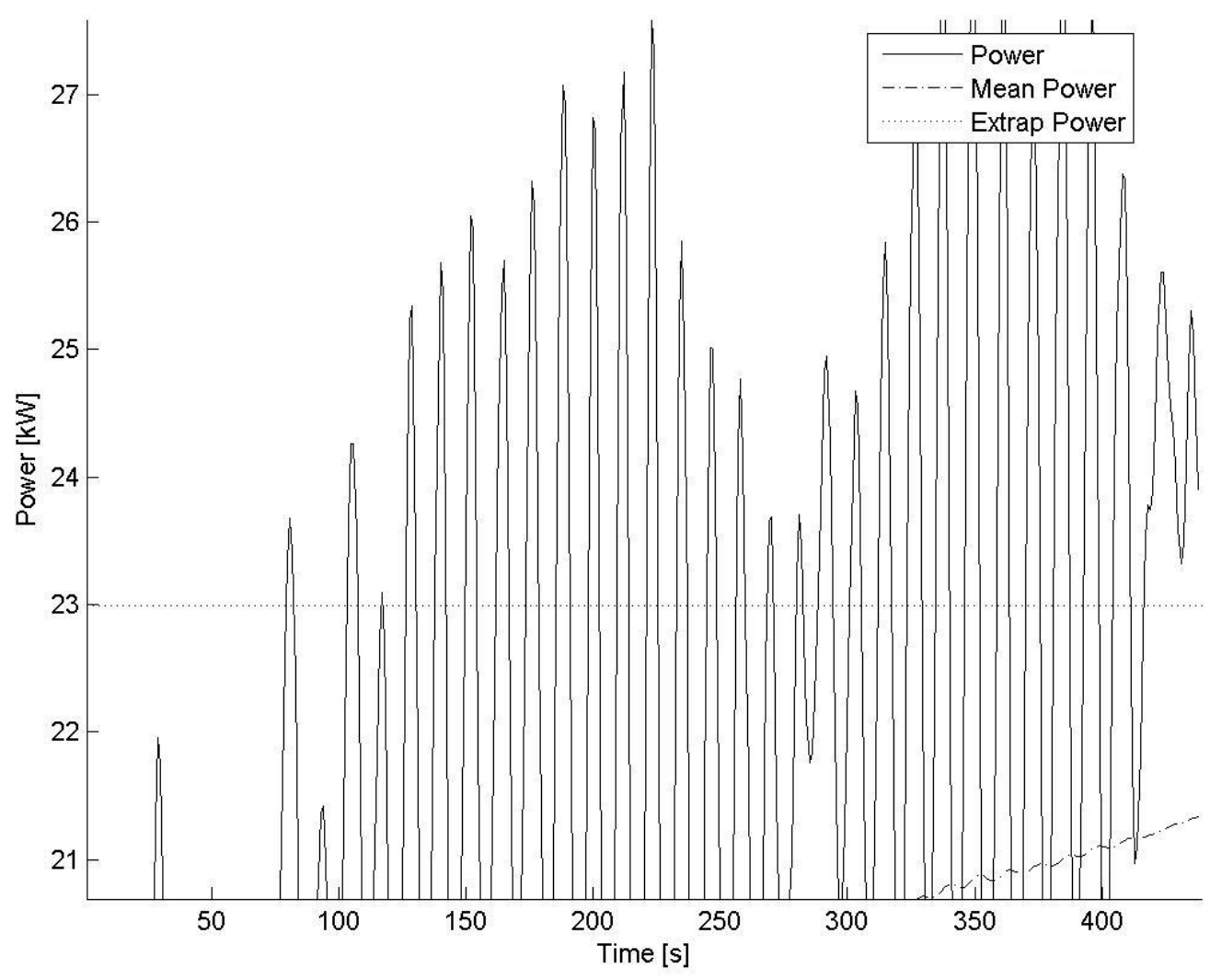

Figure 2.3: Oscillation of measured power for results of case $\left(R_{t}=0, R_{x}=0, R_{y}=0, \alpha=60^{\circ}\right)$

Scrutinizing the solution oscillations in Figure 2.3 there are 8-9 periods in a span of 100s resulting in shedding frequencies within the range of $0.08 \mathrm{~Hz}-0.09 \mathrm{~Hz}$. The range of Strouhal numbers corresponding to the shedding frequency range is $0.385 \mathrm{~Hz}-0.433 \mathrm{~Hz}$ respectively. The measured Strouhal number fall within the range given by Zdrakovich in Table 2.1 thus verifies the solution model of this study. 


\begin{tabular}{r|c|c} 
& \multicolumn{3}{c}{ Wind Variations } \\
Parameters & Low & High \\
\hline Thickness $\left(b_{x} / D_{o}\right)$ & $20 / 20$ & $40 / 20$ \\
Oblatness $\left(a / D_{o}\right)$ & $15 / 20$ & $5 / 20$ \\
Gap $\left(g / D_{o}\right)$ & $3 / 20$ & $3 / 20$
\end{tabular}

Table 2.2: Optimal parameters for different wind variations

[14]

$$
\begin{aligned}
S_{t} & =\frac{f_{r} L}{V} \\
& =\frac{0.08(20)}{4.16} \\
& =0.385
\end{aligned}
$$

After three periods and shedding frequency of $0.08 \mathrm{~Hz}$ a vortex propagates $156 \mathrm{~m}$ downstream, which is within the mesh body of influence defining the von Karman street. A vortex propagation distance was calculated by

$$
\begin{aligned}
L_{p} & =3(P) V \\
& =3(12.5) 4.16 \\
& =156 \mathrm{~m}
\end{aligned}
$$

\subsection{D Optimized BAWT Footprint}

Research of a 2D BAWT was performed by Hans Hamm [14. His model consists of a base 2D geometry where he obtained through a CFD study optimal designs that provides the greatest wind augmentation factors in cases with high and low wind incidence angles.

Figure 2.4 is Hamm's illustration of the optimal two dimensional BAWT configuration at low variations in wind incidence angles. The dimensions parameters are nondimensionalized with respect to the base diameter of the BAWT.

Hamms [14] research was a study on deriving geometries for best wind augmentation. However a configuration which produces best wind augmentation may not necessarily be the best for power production. The total power produced by wind turbines should also be taking into consideration for BAWT optimization studies. Wind speeds were shown to increase with the reduction of the gap distance but limits the wind turbines rotor diameter to produce small amounts of energy. Maximizing the rotor 


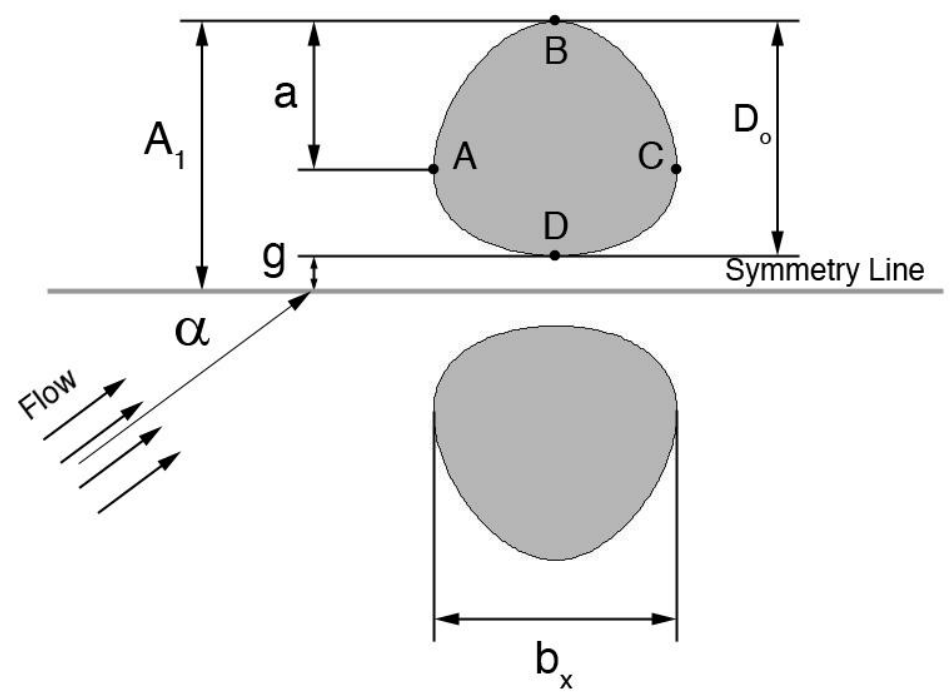

Figure 2.4: Varying parameters on a 2D building footprint 14

diameter to increase energy yields is the main factor when for wind farming and should be taken into consideration for optimizing BAWT systems.

\subsection{Statement of Purpose}

This research is intended to analyse variations of building facades with respect to three different parameters to determine their effects on power augmentation performance at various wind incidence angles. The BAWT will be modeled from a base two-dimensional building footprint where a controlled extruded three-dimensional building facade is varied under the three parameters of: taper $\left(R_{t}\right)$, forward lean $\left(R_{x}\right)$ and spread $\left(R_{y}\right)$. All BAWT configurations were analysed with wind incidence angle as a factor in the overall performance.

The goals of this research are:

1. Determine power variations of different BAWT configuration using three varying parameters such as; taper $\left(R_{t}\right)$, forward lean $\left(R_{x}\right)$, and spread $\left(R_{y}\right)$

2. Determine increase of power that can be achieved using building facades as augmenters/concentrators

3. Determine the augmentation factors for each configuration

4. Determine the best BAWT configuration for three varying wind regimes

5. Determine the overall BAWT power ratings 


\section{Chapter 3}

\section{Model and Mesh Development}

This section discusses the approach used in the development of the models and the mesh. Nineteen BAWT model configurations were created varying in shape using three parabolic parameters with associated constraints and contained within a solution domain. The grids were created to adequately defined the boundary layer along BAWT walls and the resulting turbulent flow structures downstream of the domain.

\subsection{Modeling}

Modeling was done using Design Modeller from ANSYS Workbench. The dimensional BAWT footprint generated by Hans Hamm is used as for the cross-section of the 3D model. Hamm's geometry was optimized for greatest velocity augmentation at low wind incidence angles which makes it a good starting point for this $3 \mathrm{D}$ optimization study. The $3 \mathrm{D}$ model was created by extruding and varying a $2 \mathrm{D}$ footprint geometry $100 \mathrm{~m}$ in height. Multiple BAWT configurations were generated by controlling the extrusion process using three parameters to create nineteen BAWT models. Also described is the turbine capture area to simulate the presence of a wind turbine. Lastly the size of the domain will be discussed.

\subsubsection{Domain Boundaries}

Urban landscapes are densely packed with tall residential and commercial buildings separated by roadways. The separation gaps between adjacent buildings provide corridors for wind to flow through. Since wind directions in urban landscapes are constrained to the orientations of the surrounding buildings, this study assumed low variation of wind incidence angles approaching the BAWT.

The base footprint for all contrived 3D BAWT models will be the optimized two-dimensional geometry for low varying wind incidence angles generated by the work of Hans Hamm. The optimal footprint parameters for low wind incidence are listed below:

1. a moderate thickness $\left(b_{x} / D_{o}=20 / 20\right)$ 
2. high oblateness $\left(a / D_{o}=15 / 20\right)$

3. small gap ratio $\left(g / D_{o}=3 / 20\right)$

The footprint was extruded in the vertical $z$ axis to create Wall boundaries and was controlled using three normalized parameters with respect to the base characteristic diameter $\varnothing_{o}$ of $20 \mathrm{~m}$ which was equivalent to $D_{o}$ characteristic diameter of Hans Hamm. The parameters governing the vertical taper $\left(R_{t}\right)$, forward lean $\left(R_{x}\right)$, and spread $\left(R_{y}\right)$ are:

$$
\begin{aligned}
R_{t} & =1-\varnothing_{t} / \varnothing_{o} \\
R_{x} & =x_{t} /\left(R_{t} \varnothing_{o}\right) \\
R_{y} & =y_{t} /\left(R_{t} \varnothing_{o}\right)
\end{aligned}
$$

Where $\varnothing_{t}$ is the building diameter at $100 \mathrm{~m}$ height, $\varnothing_{o}$ is the buildings base diameter, $x_{t}$ is the forward shift if the building top with respect to the building base and $y_{t}$ is the distance from the symmetry plane to the edge of the building top.

Parabolic profiles were chosen to define the vertical profile of the BAWT systems. The parabolic profile was inspired from the velocity profile of the atmospheric boundary layer (ABL) which will be discussed in subsequent sections. It was thought that a uniform velocity profile normal to the turbine capture area could be achieved in augmentation to provide uniform loading distribution to the turbines. This would require higher velocity augmentation of the low elevations and lower velocity augmentation of the high elevations of the ABL.

Prabolic inflection point is constrained to the roof top of the BAWT with respect to their coordinate systems. Constraints are imposed to limit the values of $R_{x}$ and $R_{y}$. These constraints are imposed to maintain a configuration where there is no overhang of the building over the referenced footprint. The governing system of equations defining the profiles given in Equation 3.6

$$
\begin{gathered}
P_{\text {taper }}(z)=\frac{z(200-z)}{500} \chi \\
P_{\text {lean }}(z)=\frac{z(200-z)}{500} \chi \\
P_{\text {spread }}(z)=\frac{z(200-z)}{500} \chi+3 \\
\chi=\left\{\begin{array}{lll}
R_{t} & \text { for } & 0 \leq \varnothing(z)<\varnothing_{o} \\
R_{t} R_{x} & \text { for } & 0 \leq x(z) \leq R_{t} \varnothing_{o} \\
R_{t} R_{y} & \text { for } & g \varnothing_{o} \leq y(z) \leq\left(R_{t}+g\right) \varnothing_{o}
\end{array}\right.
\end{gathered}
$$


where $\chi$ is a place holder for various cases. Parabolic profile derivation can be found in Appendix A. Figure 3.1 illustrates the use of each parameter. Figures 3.2 and 3.3 represent the geometric variations of the BAWT with respect to each parameter.

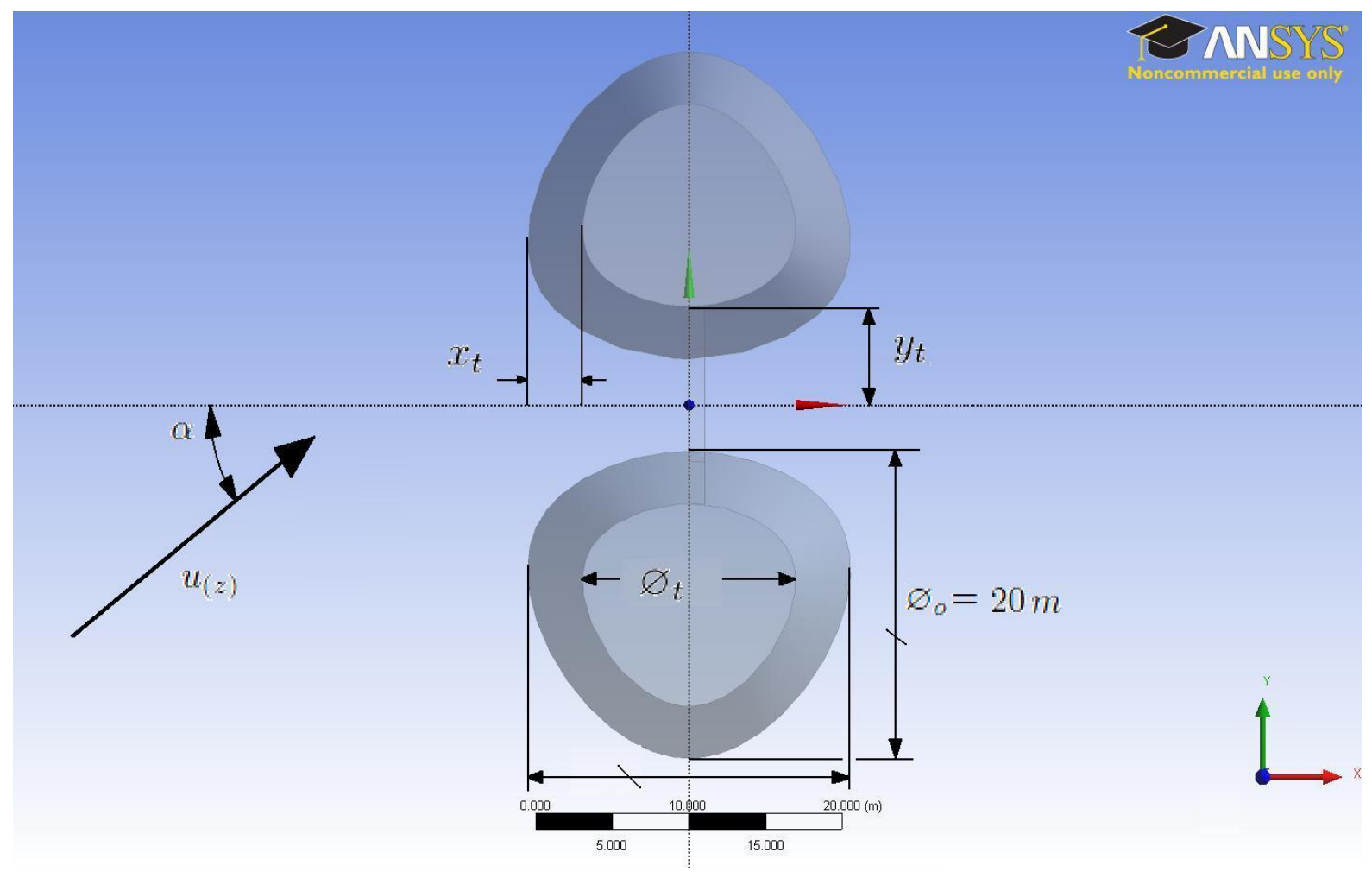

Figure 3.1: Variation of 3D BAWT parameters

The cases analysed used each building parameter at three different points of interest: minimum (0), medium (0.5) and maximum (1) values for $R_{x}$ and $R_{y} ; 0,0.33$ and 0.66 for $R_{t}$ and where subject to four incidence angles of $0^{\circ}, 20^{\circ}, 40^{\circ}$, and $60^{\circ}$. The different variations of models totalled to 76 and are shown in Table 3.1 with their respective incidence angles.

The velocity profile also defined the starting location of the modeled turbine above a referenced potential wind speed, $u_{p}$ at $10 \mathrm{~m}$ height. The potential wind speed equated to the bottom speed limit of a HAWT at $4.16 \mathrm{~m} / \mathrm{s}$ [1] and modeling turbines under this limit below $10 \mathrm{~m}$ is not appropriate. The turbine is also constrained at the augmenter throat where the maximum wind augmentation occurs. Thus it is constrained to Equation 3.6 for case $x(z)$ when $10 \mathrm{~m} \leq z \leq 100 \mathrm{~m}$ and is illustrated in Figures 3.4 and 3.5

The domain sizing is critical for the proper analysis of flow characteristics such as pressure to diffuse throughout the domain without any adverse effects from the boundaries of domain. Appropriate domain sizing for flows above Reynolds number $3.5 \cdot 10^{6}$ are suggested by Grinstein [10. The Inlet boundary is 


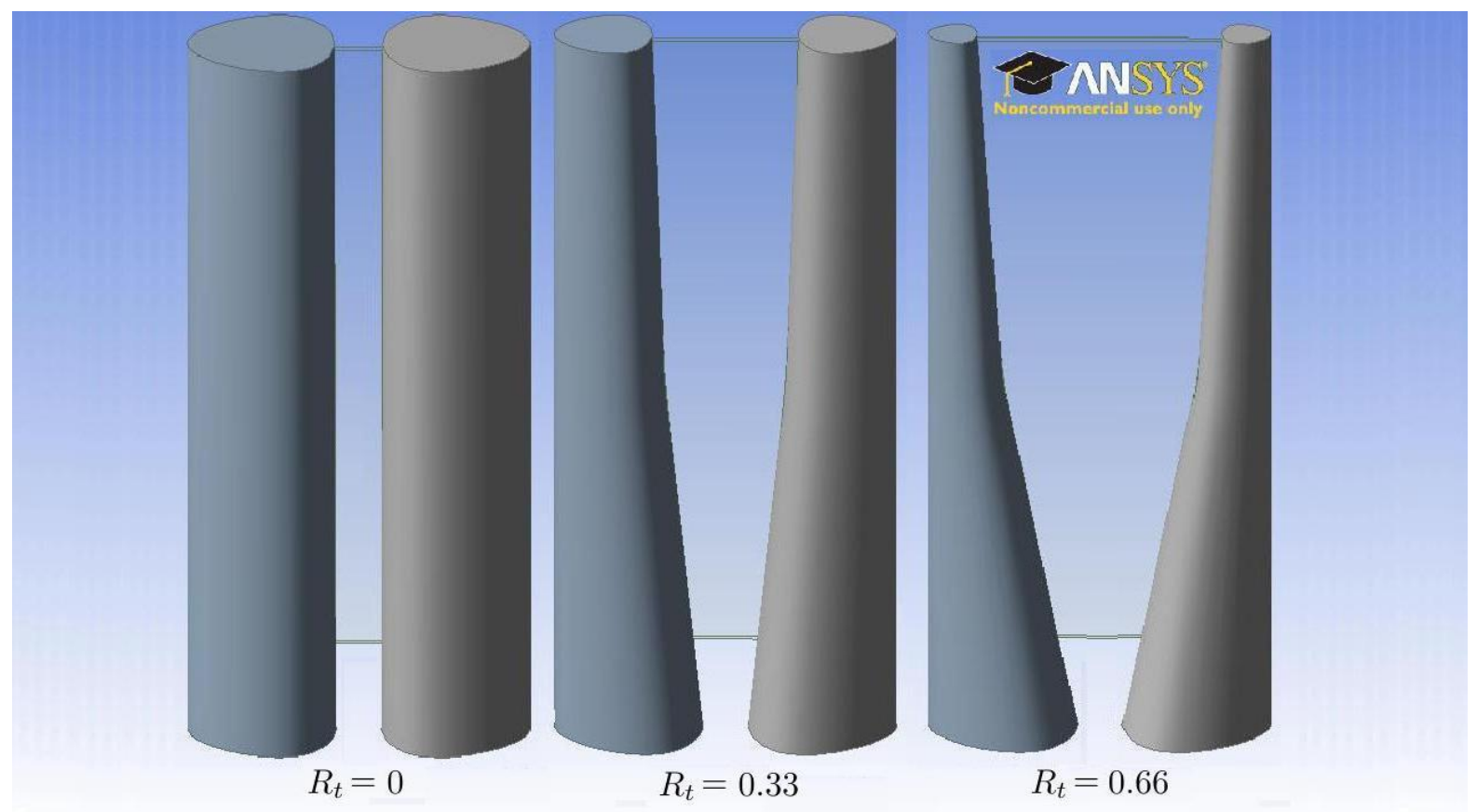

Figure 3.2: Variation of taper ratio $R_{t}$

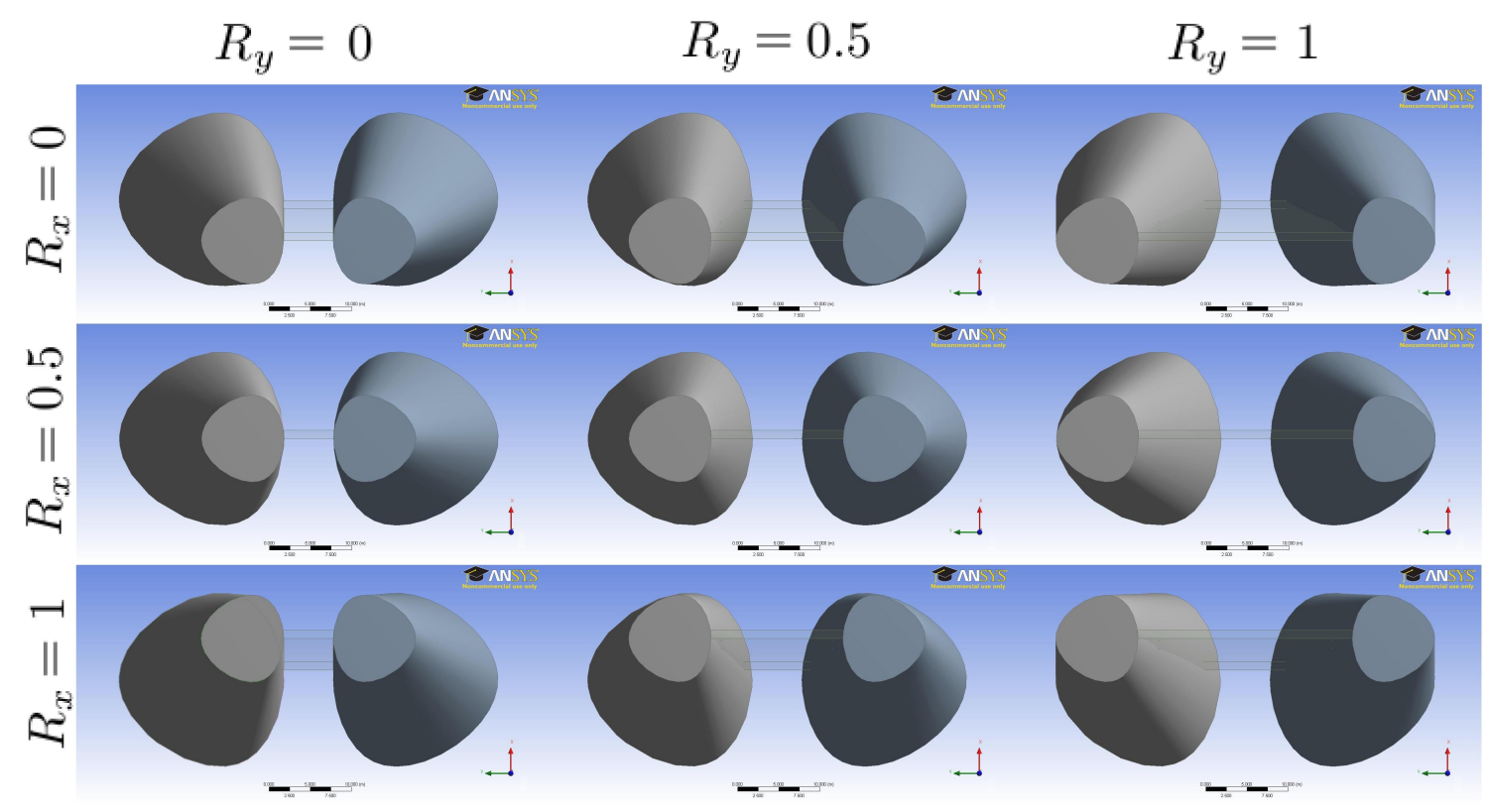

Figure 3.3: Variations of variables $R_{x}$ and $R_{y}$ 


\begin{tabular}{|c|c|c|c|c|c|c|}
\hline$R_{t}$ & $R_{x}$ & $R_{y}$ & \multicolumn{4}{|c|}{ Wind Incidence Angles $(\alpha)$} \\
\hline 0 & 0 & 0 & $0^{\circ}$ & $20^{\circ}$ & $40^{\circ}$ & $60^{\circ}$ \\
\hline \multirow{3}{*}{0.33} & & 0 & $0^{\circ}$ & $20^{\circ}$ & $40^{\circ}$ & $60^{\circ}$ \\
\hline & 0 & 0.5 & $0^{\circ}$ & $20^{\circ}$ & $40^{\circ}$ & $60^{\circ}$ \\
\hline & & 1 & $0^{\circ}$ & $20^{\circ}$ & $40^{\circ}$ & $60^{\circ}$ \\
\hline \multirow{3}{*}{0.33} & & 0 & $0^{\circ}$ & $20^{\circ}$ & $40^{\circ}$ & $60^{\circ}$ \\
\hline & 0.5 & 0.5 & $0^{\circ}$ & $20^{\circ}$ & $40^{\circ}$ & $60^{\circ}$ \\
\hline & & 1 & $0^{\circ}$ & $20^{\circ}$ & $40^{\circ}$ & $60^{\circ}$ \\
\hline \multirow{3}{*}{0.33} & & 0 & $0^{\circ}$ & $20^{\circ}$ & $40^{\circ}$ & $60^{\circ}$ \\
\hline & 1 & 0.5 & $0^{\circ}$ & $20^{\circ}$ & $40^{\circ}$ & $60^{\circ}$ \\
\hline & & 1 & $0^{\circ}$ & $20^{\circ}$ & $40^{\circ}$ & $60^{\circ}$ \\
\hline \multirow{3}{*}{0.66} & & 0 & $0^{\circ}$ & $20^{\circ}$ & $40^{\circ}$ & $60^{\circ}$ \\
\hline & 0 & 0.5 & $0^{\circ}$ & $20^{\circ}$ & $40^{\circ}$ & $60^{\circ}$ \\
\hline & & 1 & $0^{\circ}$ & $20^{\circ}$ & $40^{\circ}$ & $60^{\circ}$ \\
\hline \multirow{3}{*}{0.66} & & 0 & $0^{\circ}$ & $20^{\circ}$ & $40^{\circ}$ & $60^{\circ}$ \\
\hline & 0.5 & 0.5 & $0^{\circ}$ & $20^{\circ}$ & $40^{\circ}$ & $60^{\circ}$ \\
\hline & & 1 & $0^{\circ}$ & $20^{\circ}$ & $40^{\circ}$ & $60^{\circ}$ \\
\hline \multirow{3}{*}{0.66} & & 0 & $0^{\circ}$ & $20^{\circ}$ & $40^{\circ}$ & $60^{\circ}$ \\
\hline & 1 & 0.5 & $0^{\circ}$ & $20^{\circ}$ & $40^{\circ}$ & $60^{\circ}$ \\
\hline & & 1 & $0^{\circ}$ & $20^{\circ}$ & $40^{\circ}$ & $60^{\circ}$ \\
\hline
\end{tabular}

Table 3.1: Various model cases

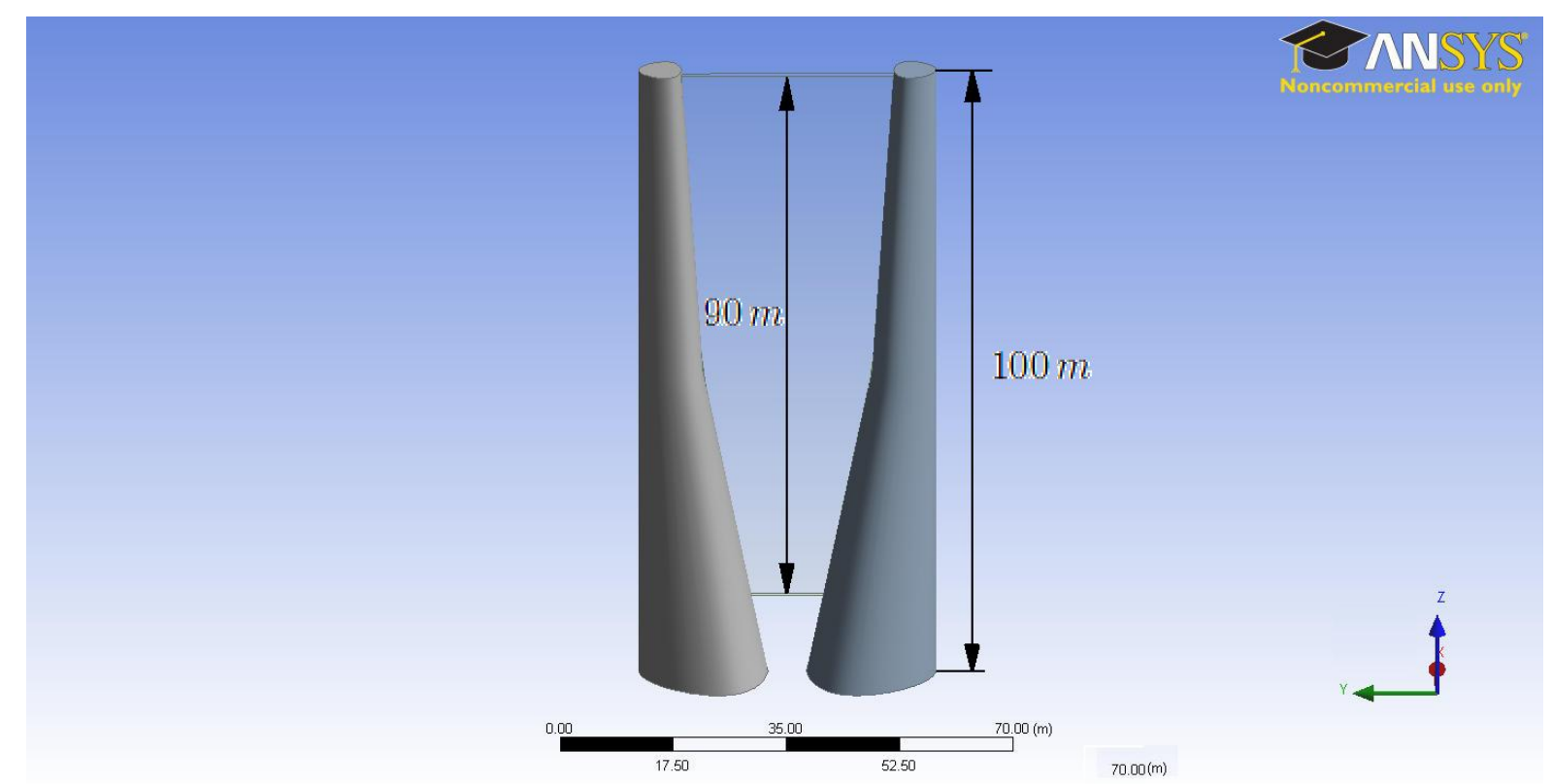

Figure 3.4: Front view of BAWT and simulated turbine capture area 


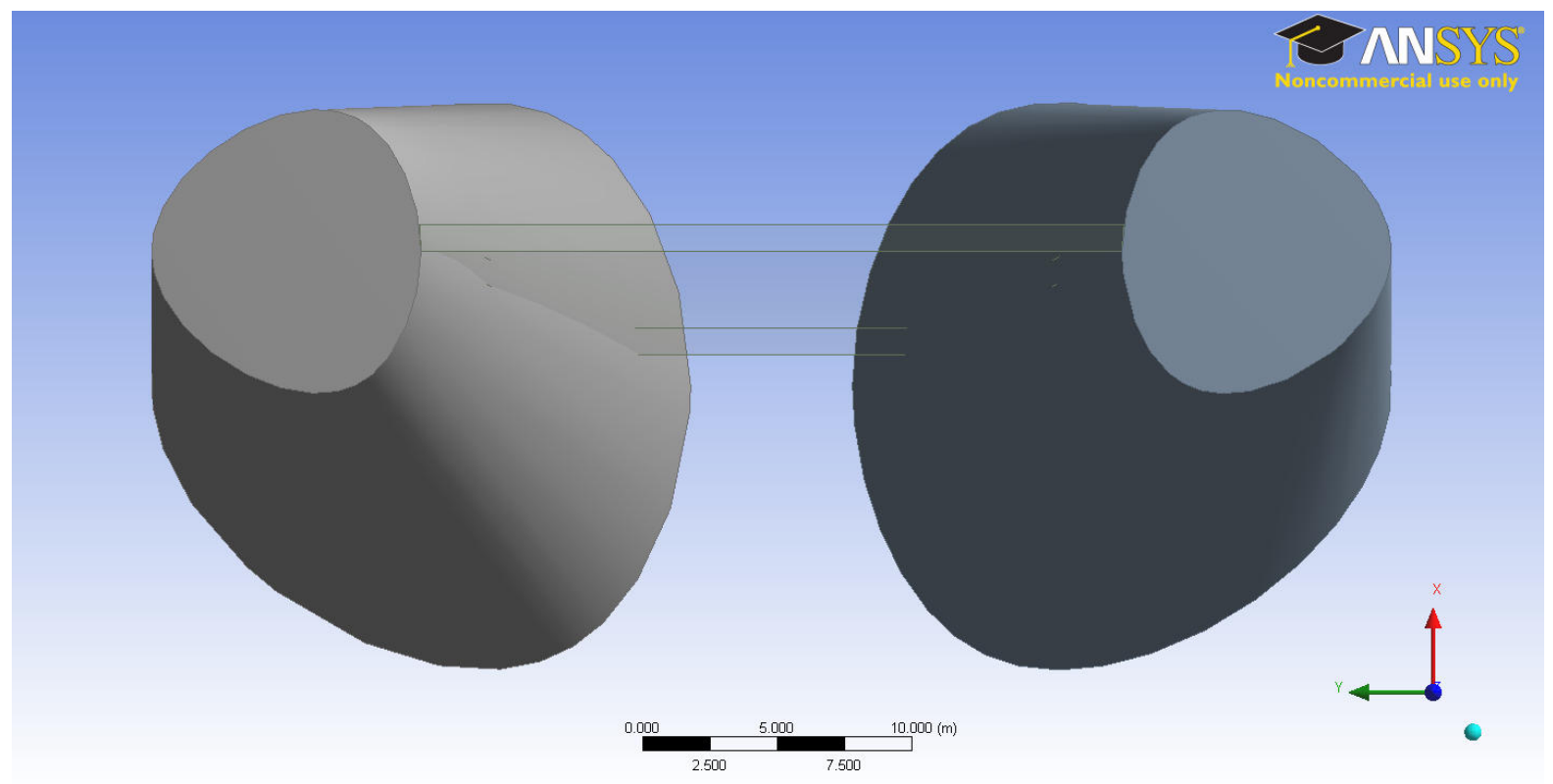

Figure 3.5: Top view of turbine spans across and extends height of the BAWT

located $200 \mathrm{~m}$ upstream, Outlet boundary is $400 \mathrm{~m}$ downstream, Side boundaries are $200 \mathrm{~m}$ cross-stream and Top is $300 \mathrm{~m}$ above the boundary Ground. Domain sizing is illustrated in Figure 3.6 and Figures 3.7 3.8 show the domain and turbine boundary locations.

\subsubsection{Bodies of Influence}

The fidelity of the solution of the BAWT configurations is dependent on the mesh resolution of the defining von Karman street. The latter occurs from the resulting shedding and propagation of vortex structures downstream from bluff bodies. Grid resolution must define these turbulent structures well enough to accurately model the pressure cascade of the von Karman street. Tapered cylinders produces varying vortex structures which are described to be a linear function of taper by Piccirrillo and Van Atta [11, 12, 21]. Two bodies of influence will refine the mesh to resolve the varying shed vortex scales.

The size of the body of influence to define the von Karman street from a cylinder for Reynolds numbers above $5.5 \cdot 10^{5}$ was suggested to be three times the cylinder diameter by Grinstein [10. A coarse body of influence was defined by a sphere of diameter $120 \mathrm{~m}$ extending its centroid from the Ground at the center of the BAWT extending to $100 \mathrm{~m}$ in height, and spanning the length of the Domain downstream $400 \mathrm{~m}$. The fine body of influence is defined by a sphere of $80 \mathrm{~m}$ also extending its centroid from the Ground at the center of the BAWT extending 100m in height and spanning 250m downstream. Figure 3.9 shows the Bodies of Influence defining the von Karman vortex street. 

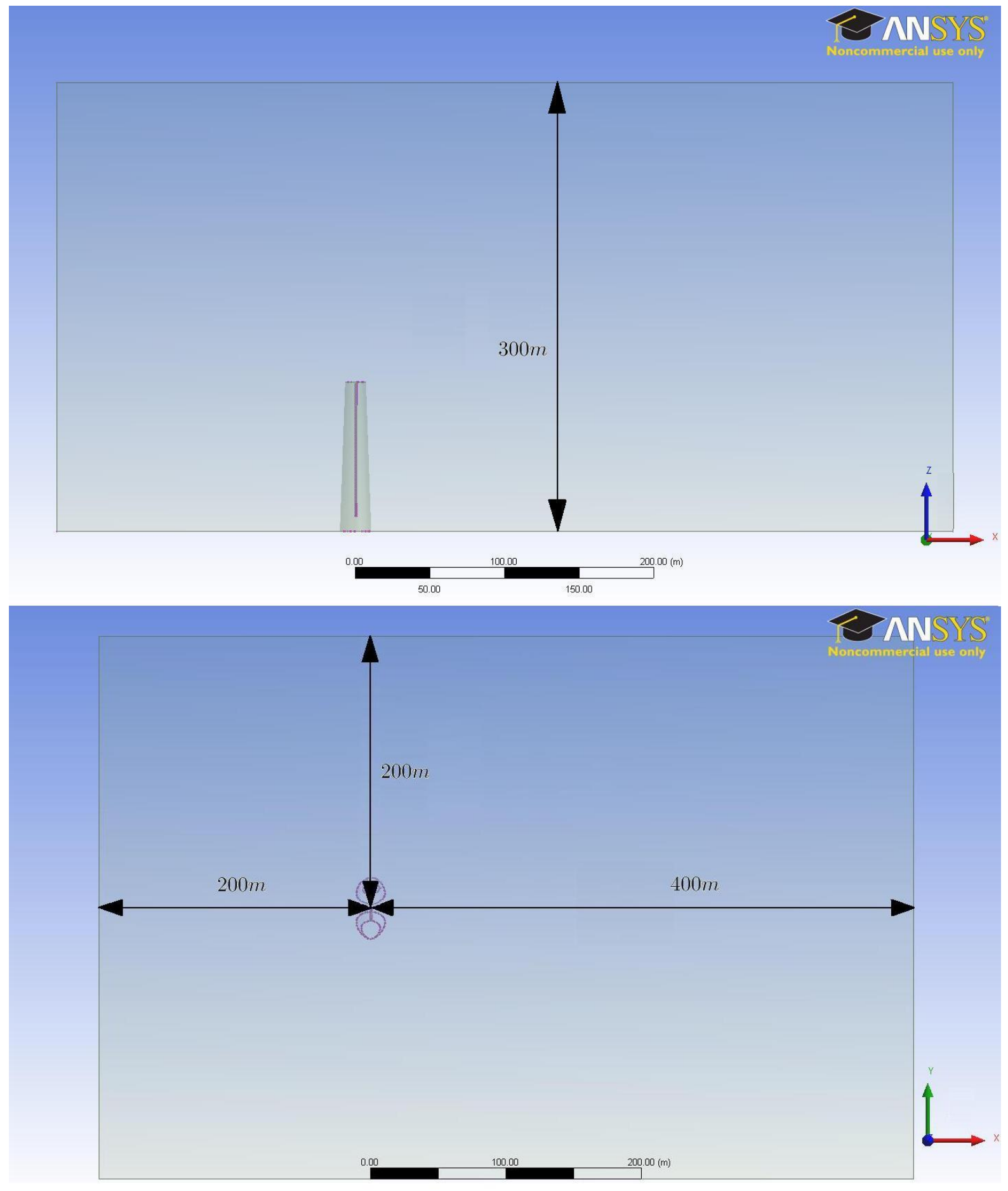

Figure 3.6: Side and top views of the domain 


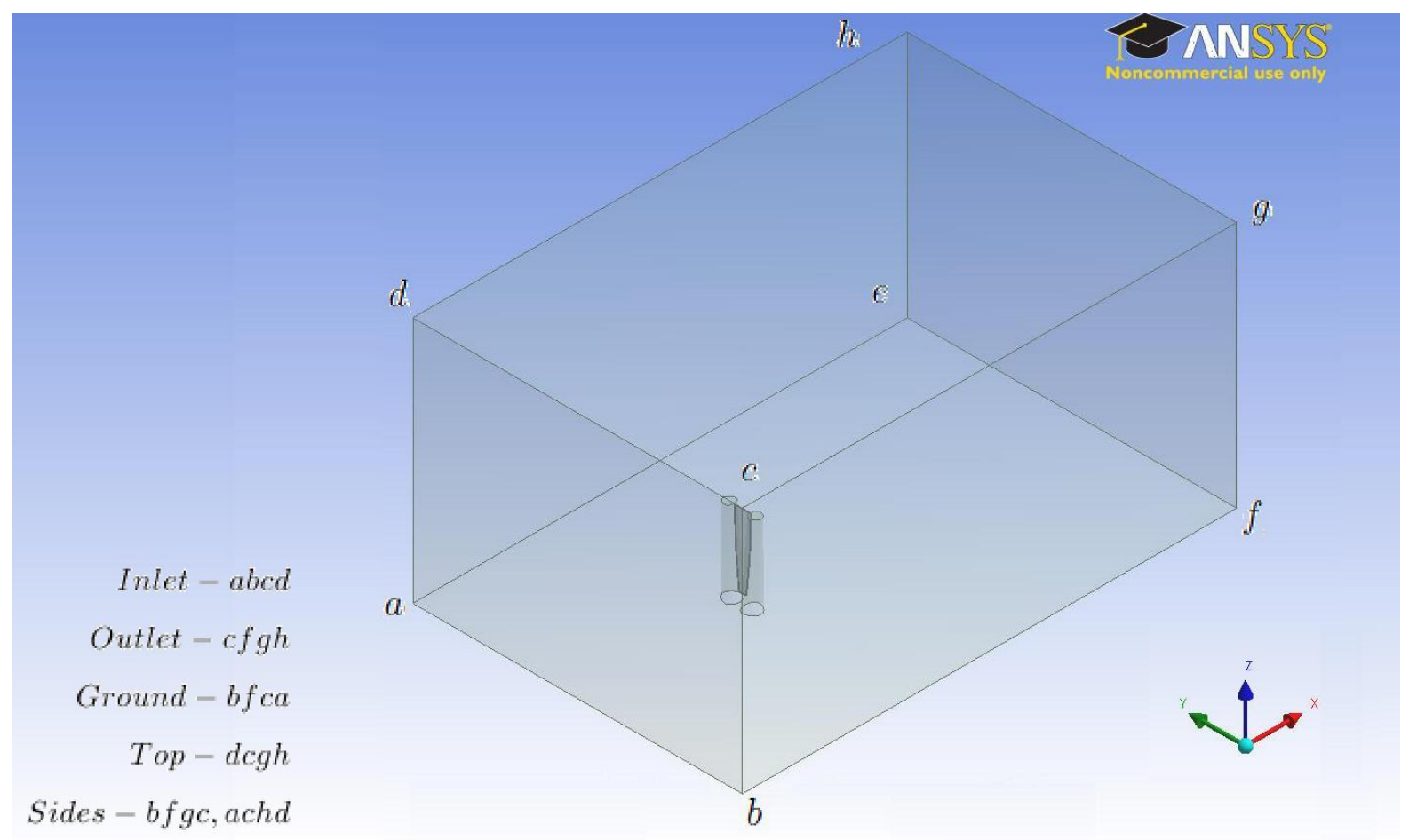

Figure 3.7: Isometric view of the domain 


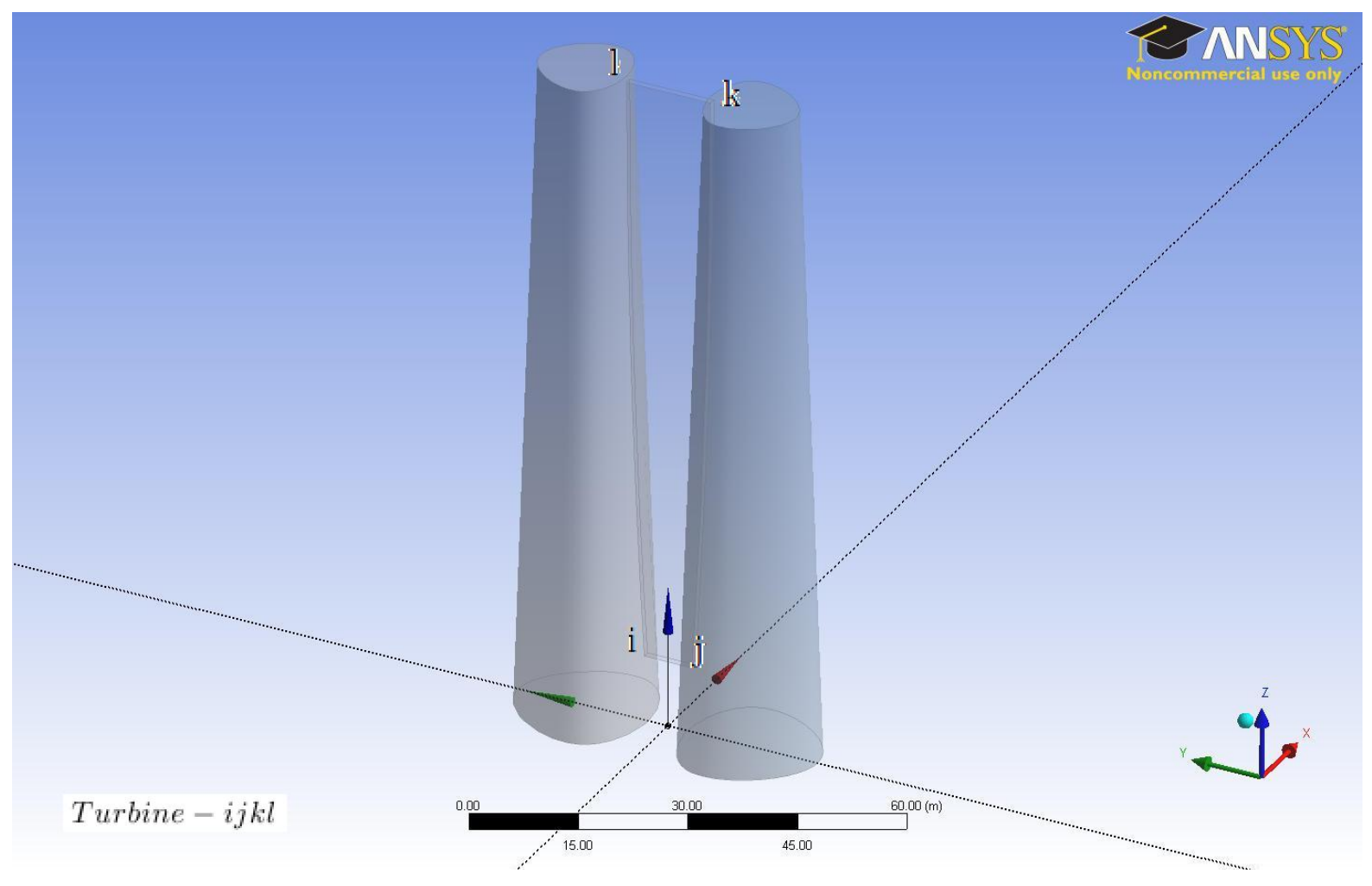

Figure 3.8: Close view of BAWT system with plain simulating turbines 


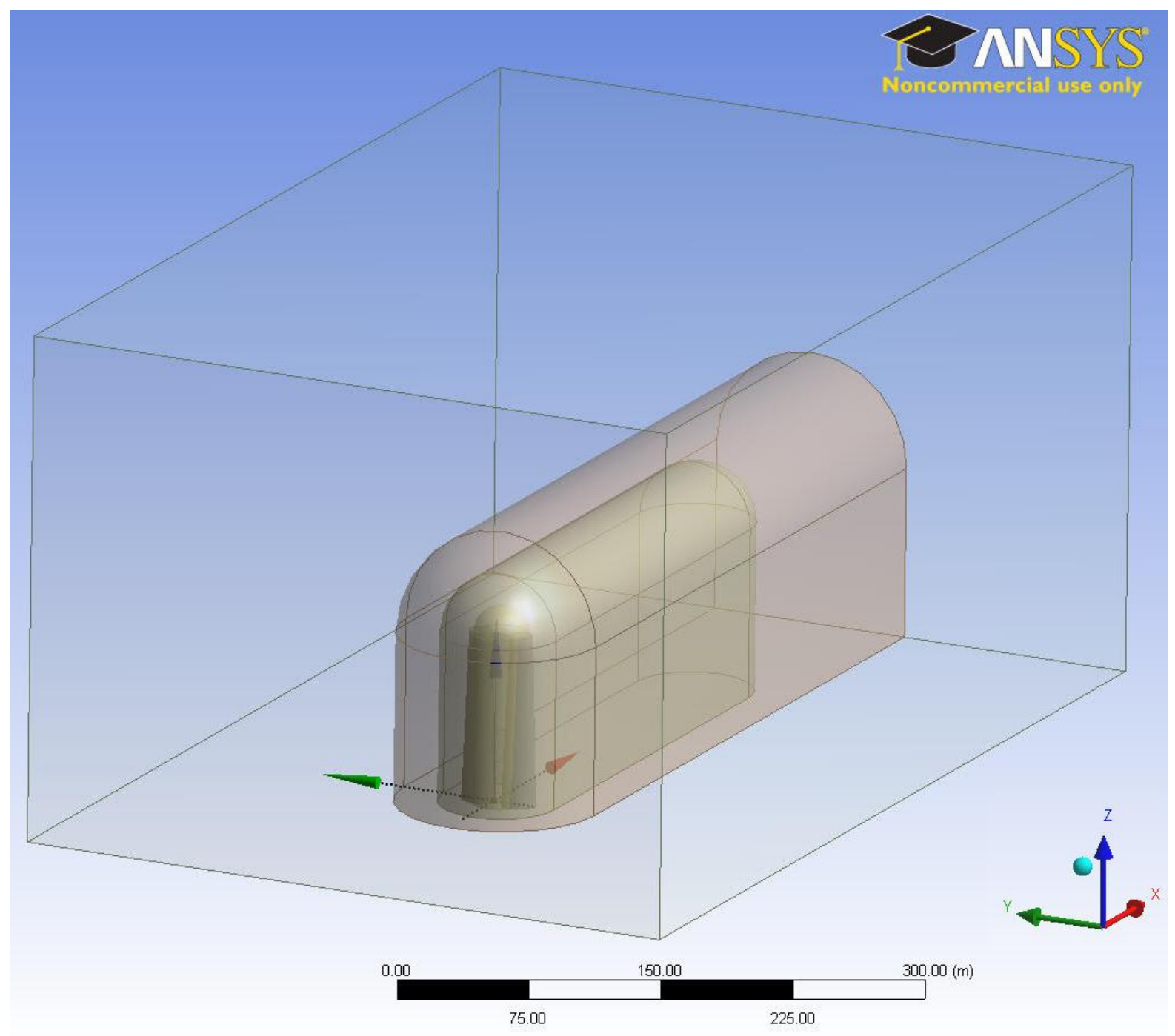

Figure 3.9: Bodies of influence defining the vortex street defined 


\subsection{Meshing}

CFD requires the computational domain to be discretized into elements for the solver to apply the discrete RANS fluid equations at each element interface. ANSYS MESH was used to discretize the solution domain around the various BAWT geometries.

An unstructured grid system was chosen for its suitability to model complex turbulent flow structures within the von Karman street [16. Modeling of the von Karman street relies on accurate modeling of the adverse pressure gradients of vortex shedding and requires adequate boundary layer resolution on the Wall boundaries. A near-wall model was used to approximate transition to laminar boundary layer where boundary layer accuracy can be reduced to $y^{+}$values from 1-10. The boundary layer was defined at the first prism height, $\Delta y$ of $0.001 \mathrm{~m}$ with 25 inflated prism layers at an expansion ratio of 1.2 .

\subsection{Inflation Layers}

Inflation layers were used to capture the velocity gradient of the boundary layer developed by shear friction occurring from a no-slip wall condition of the domain Walls. Also it is critical to capture the laminar separation with appropriate mesh refinement to capture the separation bubble from the shed vortex.

To resolve flow calculations to a degree of accuracy, FLUENT uses an enhanced wall treatment for the selected $k-\omega$ shear stress transport (SST) model. This wall treatment uses a near-wall approximation method predicts the boundary's viscous and transition layers. This reduces the need to extensively refine the mesh near a Wall boundary to capture the boundary layer profile, and also reduces computational cost. Figure 3.10 shows inflation layers surrounding a cross section of the buildings facade,

$$
y^{+}=\frac{\rho u_{\tau} y_{p}}{\mu}
$$

where $u_{\tau}=\sqrt{\frac{\tau_{\omega}}{\rho_{\omega}}}$ is the friction velocity, and $\tau_{\omega}$ is the shear stress and $\rho_{\omega}$ is the density of the fluid at the surface wall, $y_{p}$ is the displacement of the first adjacent nodal point $P$ from the wall and $\rho$ is the density of the fluid.

For the $k-\omega$ (SST) turbulence model, Fluent recomends when using the enhanced wall treatment to obtain a $y^{+}$value of the first adjacent nodes to be within $1 \leq y^{+} \leq 5$. FLUENT approximates the first inflation layer height with Equation 3.8 .

$$
\Delta y=L y^{+} \sqrt{80} R e^{(-13 / 14)}
$$

where $y^{+}$is the targeted value of the first adjacent node, $L$ is the structural cord length and $R e$ is 


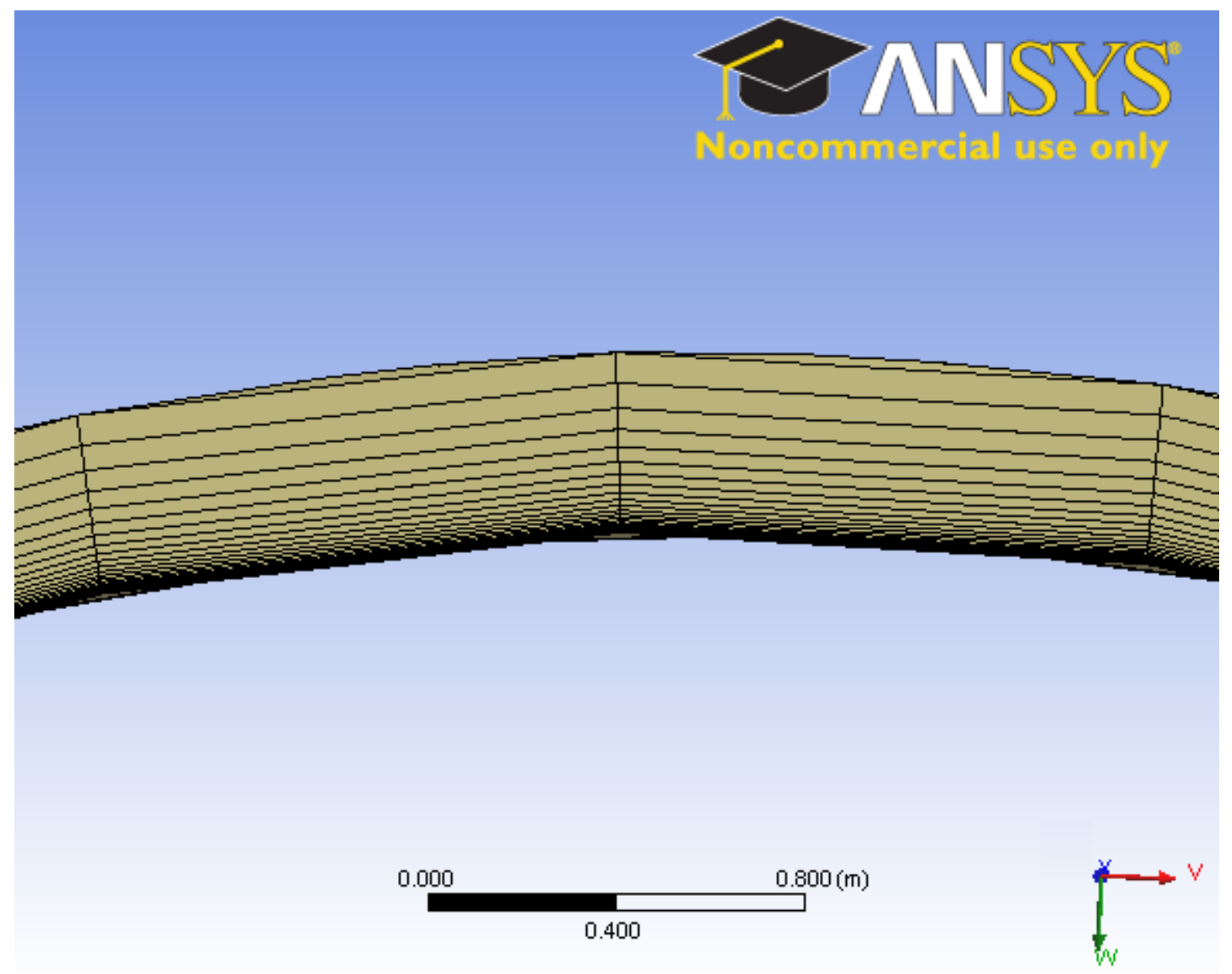

Figure 3.10: Inflation layers against building facade 
the average flows Reynolds number. Reynolds number is defined as

$$
R e=\frac{\rho V L}{\mu}
$$

A $y^{+} \approx 10$ was used to define the first non-dimensional off-wall spacing. The reference $y^{+}$of 5 was not used for the purpose of reducing mesh size and computational time. The first node spacing off the wall with respect to a Reynolds number of $5.5 \cdot 10^{6}$ is calculated to be,

$$
\begin{aligned}
\Delta y & =20(10) \sqrt{80}\left(5.5 \cdot 10^{6}\right)^{(-13 / 14)} \\
& =0.000984 \\
& \approx 0.001 m
\end{aligned}
$$

The number of inflation layers was selected in FLUENT MESH operation to define the resultant inflation around the buildings. The final inflation layer thickness of $0.1 \mathrm{~m}$ was assumed to generate a cell height to length ratio of 10 to provide good solution transition from the inflation layers to the domain elements sized at $1 \mathrm{~m}$. Inflation layer height was increased by the standard growth factor of 1.2 , thus the number of inflation layers is calculated by Equation 3.10 .

$$
\begin{aligned}
h_{n}= & \Delta y(1.2)^{n} \\
\left(\frac{h_{n}}{\Delta y}\right) & =(1.2)^{n} \\
\ln \left(\frac{h_{n}}{\Delta y}\right) & =n \ln (1.2) \\
n & =\frac{\ln \left(\frac{0.1}{0.001}\right)}{\ln (1.2)} \\
& \approx 25
\end{aligned}
$$

Thus the inflation applied to the building in this study uses 25 layers, with a starting inflation height of $0.001 \mathrm{~m}$ and a growth factor of 1.2 . Figure 3.11 shows a plot of the $y^{+}$values up along the building height for transient solutions. The $y^{+}$values in Figure 3.11 are shown to be within the reasonable range which FLUENT determines to be good for near-wall approximations to take effect. 


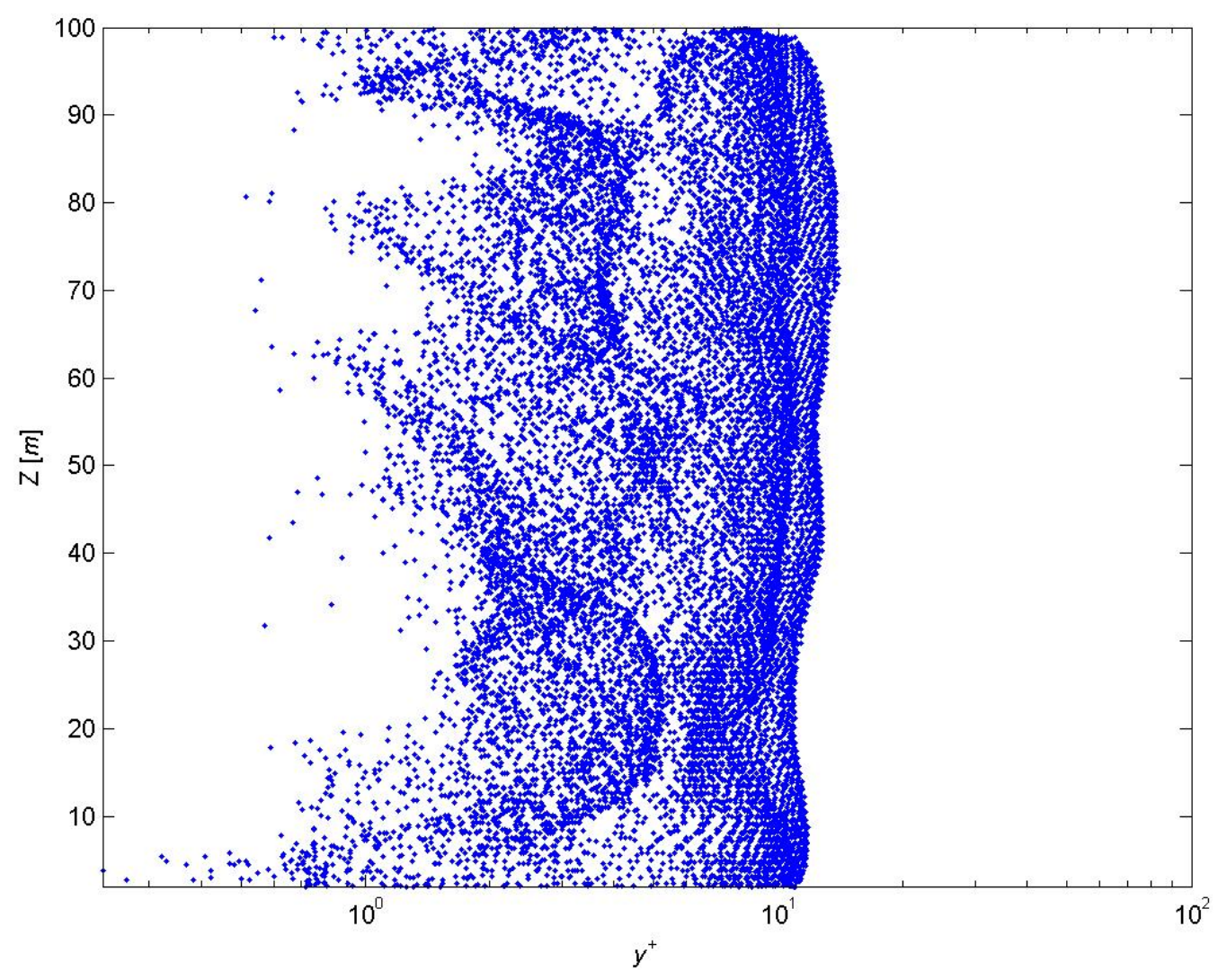

Figure 3.11: Unwrapped building surface's $y^{+}$values along building height 


\subsection{Sponge Layer}

For transient flows, turbulence poses problems were the residuals of the scalar quantities at the outlet boundary can reflect back into the solution domain by the solver. Grinstein suggests the use of a sponge layer to manipulate the multidimensional vertical structures to approach normal to the Outlet domain [10. A single 5m inflated prism layer called a 'sponge layer' shown in Figure 3.12 was used.

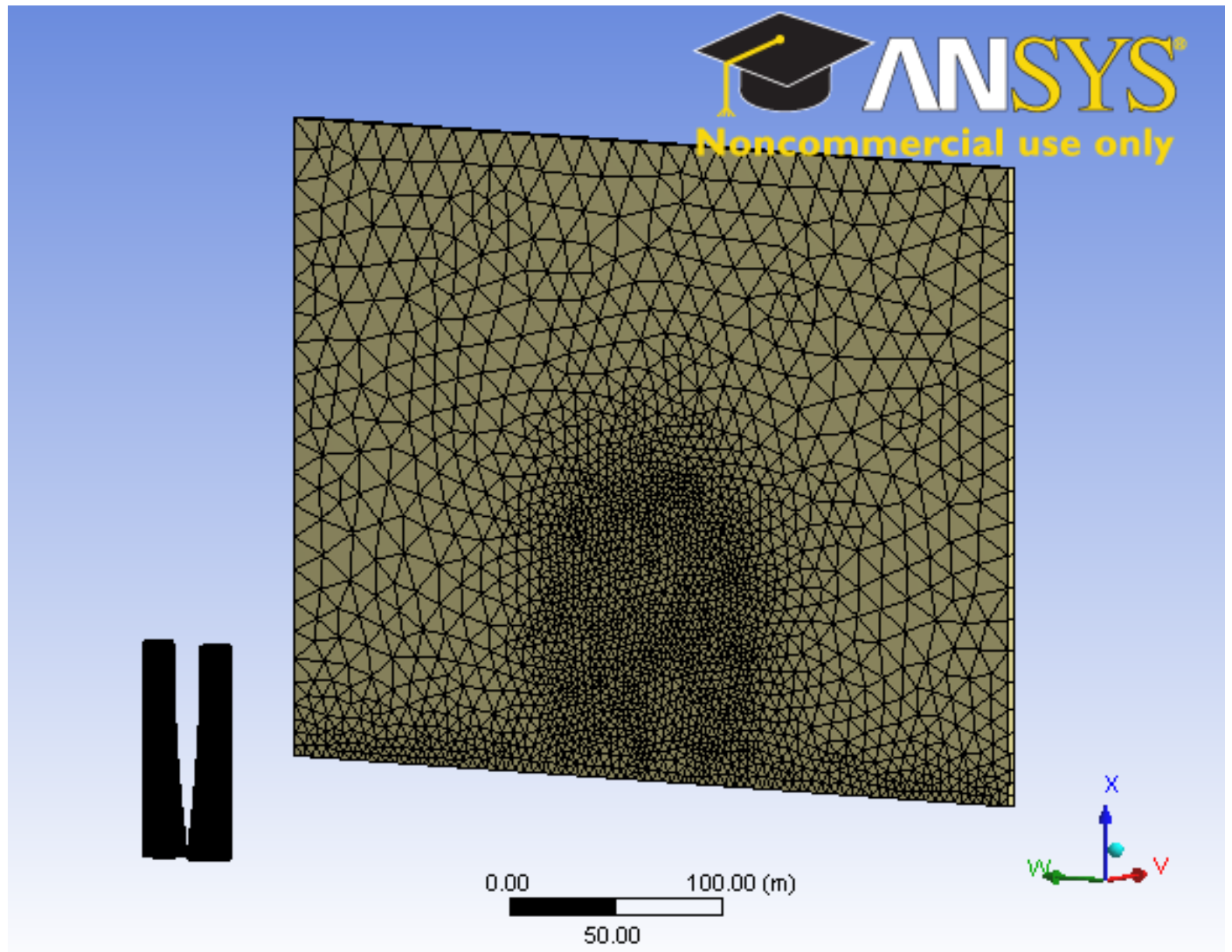

Figure 3.12: Sponge layer defining the outlet boundary

\subsection{Element sizing}

Face and element sizing specified for the model domains and boundaries were obtained from a grid study analysis. The resulting element sizings are shown in Table 3.2. A surface mesh of the Walls, Turbine and Ground is shown in Figure 3.13 It can be seen the Fine Body of Influence refines the face sizing of 


\begin{tabular}{rc} 
Bodies & Size $(\mathrm{m})$ \\
\hline Domain & 15 \\
Coarse body of influence & 5 \\
Fine body of influence & 2.5 \\
Turbine & 1 \\
& \\
Faces & Size $(\mathrm{m})$ \\
\hline Ground & 5 \\
Walls & 1
\end{tabular}

Table 3.2: Element size controls

the Ground at the base and downstream of the BAWT to define the vortex path. Figure 3.14 illustrates the element growth factor produced from the bodies of influence.

\subsection{Grid and Time Step Study}

The accuracy a numerical simulation has is dependent on the grid resolution. Through parametric studies of grid resolutions, insight is gained into when the physics modeled becomes independent of grid spacing and does not change with increased grid refinement.

Steady state simulations are conducted for this preliminary grid independence study. This study conducted tests using four grids ranging in density of $0.66 \cdot 10^{6}-3.9 \cdot 10^{6}$ elements. The power within the wind at the Turbine plane was the monitored parameter in determining solution convergence.

This study looks into the concentrator affects of a BAWT under time averaged conditions. Transient simulations are used to perform time step simulations where the solution is found for a series of time-step for a specified time interval. Just like the grid independence study, a time-step study is performed with the derived grid from the grid independence study. The three time-step sizes used in this study were $0.1 \mathrm{~s}, 1 \mathrm{~s}$ and $10 \mathrm{~s}$.

Lastly a verification of the resultant time-step was used in conjunction with the parametric grid study under a transient simulation. This study will verify mesh independence with the resultant time-step.

\subsubsection{Steady State Grid Convergence}

The grid study used four meshes with all the same controls applied to them but are different in their scaling parameters. This parameter allows all elements to be adjusted in scale, varying in the $x, y$, and $z$ directions.

The initial mesh was scaled by factors of $0.5,0.75,1$ and 1.4 where a scaling less than 1 represents a stretch factor, and a factor above 1 is shrinkage factor. Each scaled mesh was tested until convergence 


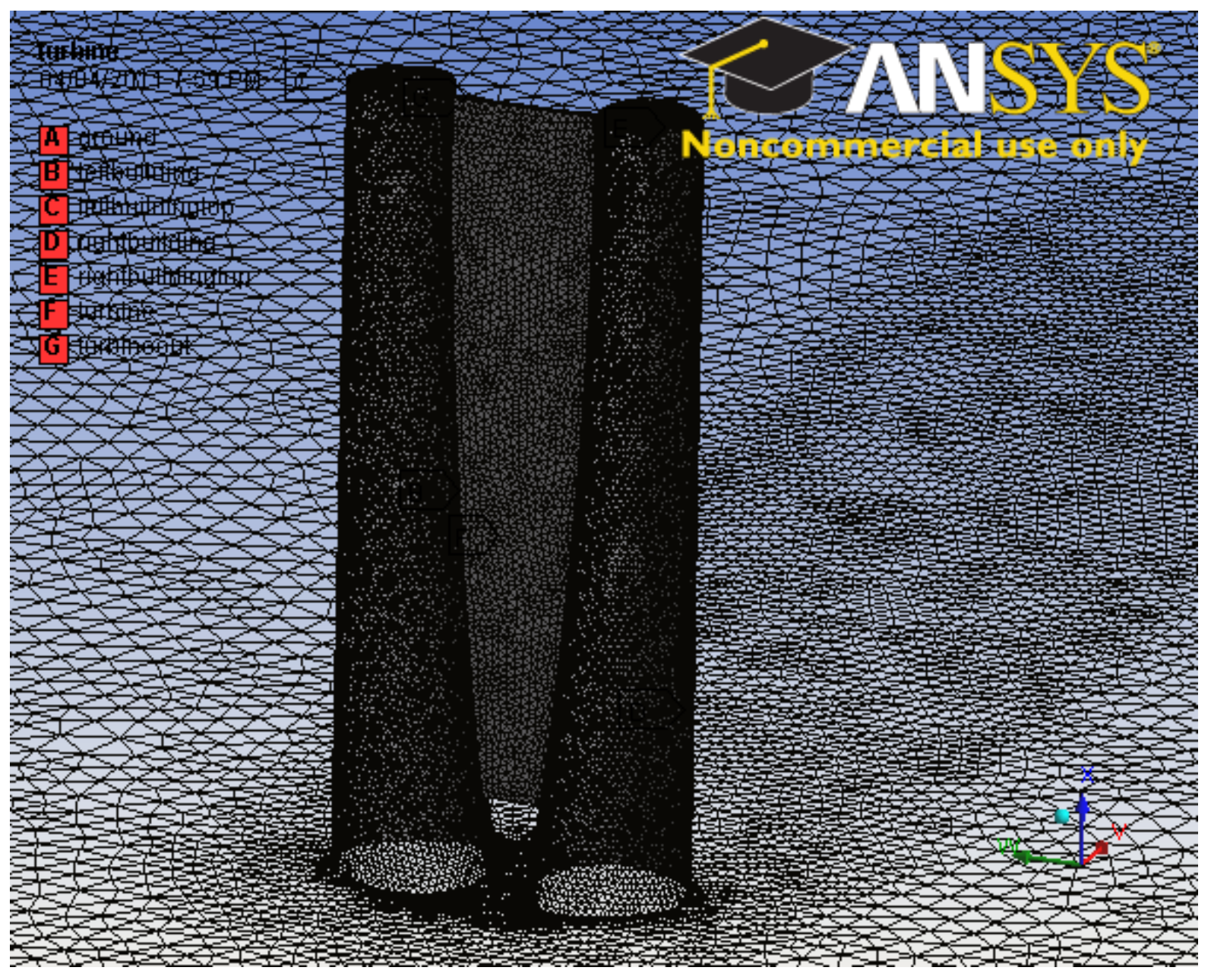

Figure 3.13: Surface mesh of Walls, Turbine and Ground 


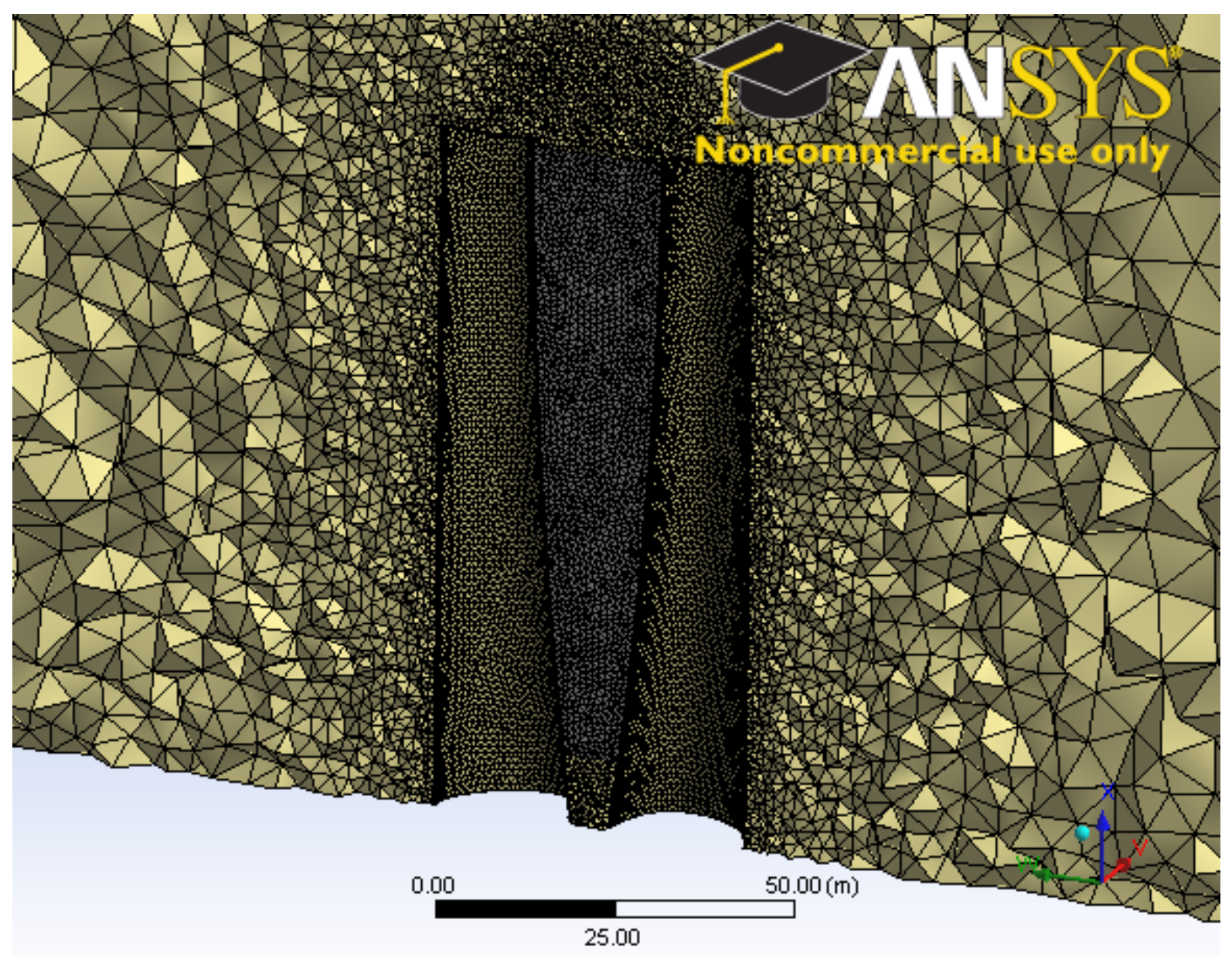

Figure 3.14: Mesh sliced lengthwise across Turbine 
of the augmented power of the BAWT was reached. The resultant augmented powers plotted with respective to their mesh element densities. A Richardson extrapotation was performed using Matlab where the asymtote of the converging power solution is found. The extrapolated solution was then used as base point in determining the accuracy of the solution to the respective mesh sizings.

Figure 3.15 shows the comparative study of the four scaled meshes where the solutions appear to describe an asymptotic convergence of the augmented power to an extrapolated power of $346.9 \mathrm{~kW}$.

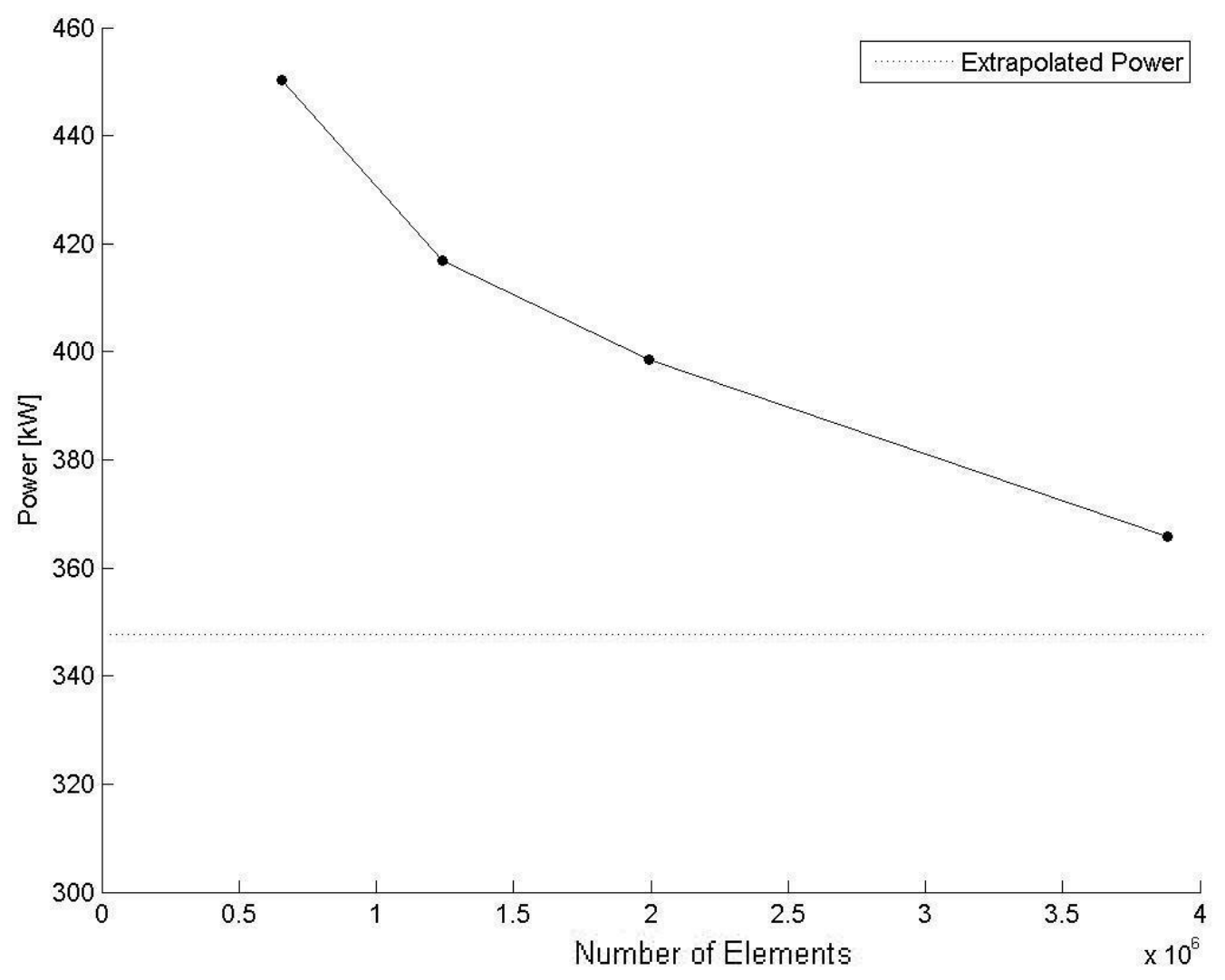

Figure 3.15: Steady state mesh convergence

Resultant deviations of the augmented power ratios compared to the extrapolated power ratio from the cases are shown in Table 3.3 . Using a steady state solution it is shown the finest mesh has a deviation of $5.45 \%$ from the extrapolated power which is too high to conclude the solution of the discretized domain to be grid dependent. A solution is considered grid independent if there less than $1 \%$ deviation between the measured power and the extrapolated value.

The steady state augmented powers are noted to be high and unrealistic. This is due from the assumed steady state solution and the values were approximated with no transient fluctuations affecting turbulence in the flow resulting in the over evaluations of scalar variables. Thus a transient grid study 


\begin{tabular}{c|r|c} 
Mesh & Elements & $\begin{array}{c}\text { Deviations from } \\
\text { Extrapolated Result }\end{array}$ \\
\hline 1 & 656,637 & $29.8 \%$ \\
2 & $1,241,258$ & $20.2 \%$ \\
3 & $1,994,056$ & $14.9 \%$ \\
4 & $3,883,171$ & $5.5 \%$
\end{tabular}

Table 3.3: Steady state grid convergence and associated errors

was conducted to resolve this issue.

\subsection{Time Step Study}

Conversely, just like the grid convergence a study in time step size was done such that the transient turbulent behaviour and von Karman street is modeled and resulting turbine back pressure is simulated. A time-marching or dual-time scheme method used by ANSYS FLUENT which finds a converged steady state solution at the respective flow time and then advances the solution by the time step and converges another steady state solution for that time step, so on so forth.

Eddies are formed the flow through viscous shearing stemming from the flows surface friction form the ground and buildings. Eddies formed by the buildings separate from the surface, known as vortex shedding and migrate downstream with the flow. Length and time scales are needed to be resolved to obtain adequate turbulent characteristics within the flows discretized domain. Thus a time step study was performed using the previous resolved mesh number 4 of the grid study.

The effects of time step size where performed using three time step sizes of $0.1 \mathrm{~s}, 1 \mathrm{~s}$ and $10 \mathrm{~s}$. The convergence of power ratio was used to determine the appropriate time step. It should be noted the case of time step of $0.1 \mathrm{~s}$ had not completed to a convergence value less than $0.5 \%$ of its mean extrapolated power ratio in over a hundred processing hours. Due to the vast amount of time needed to complete a full study using 0.1s time step, it was not considered. Conversely, solution convergence was not obtained using a 10s time step and fluctuations of the measured power ration varied $10 \%$, thus is inappropriate for this study.

The case using $1 \mathrm{~s}$ time step resolved to a converged value and the solution remained within a $0.5 \%$ deviation range for several hundred time step. Logically the 1s time step seemed most appropriate to begin with since the average element size to the discretized solution is of $1 \mathrm{~m}$ and which are of similar size in scale. The processing time for solution convergence is shown in Table 3.4 Within the maximum alloted processing time Sharcnet provided was 100h. Using a time step of $0.1 \mathrm{~s}$ and a grid size of $3.9 \cdot 10^{6}$ elements, the processing did not finish (DNF). For the larger time step of 10s, the solution did not converge (DNC).

The convergence time for the cases of one second time steps was around $24 \mathrm{~h}$ of processing time with the use of 8 way parallel processing on Sharcnet's Guppy system. Guppy uses Inlet Xeon cores with 2.67 GHz and 24.0 GB RAM and cores are connected by a QDR InfiniBand. A sample read out from 


\begin{tabular}{c|c|c}
$\begin{array}{c}\text { Time Step } \\
\text { Size }[\mathrm{s}]\end{array}$ & $\begin{array}{c}\text { Processing } \\
\text { Time }[\mathrm{h}]\end{array}$ & $\begin{array}{c}\text { Power } \\
{[\mathrm{kW}]}\end{array}$ \\
\hline 0.1 & $>100$ & $\mathrm{DNF}$ \\
1 & $<20$ & 106.2 \\
10 & $<4$ & DNC
\end{tabular}

Table 3.4: Convergence of power with respect to time step size

the generated output file FLUENT illustrates the processing time needed for one solution to complete.

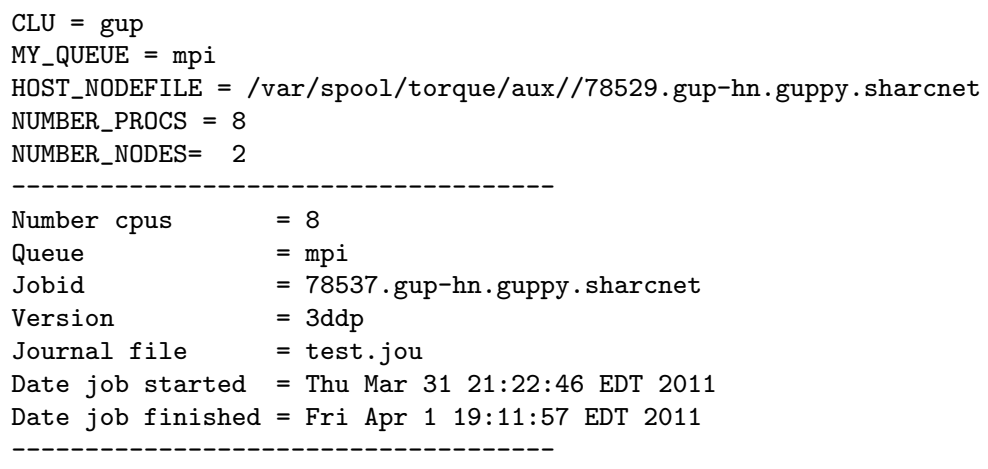

\subsection{Grid-Time Step Study}

Simulations were tested for a length of time until results showed a steady mean of transient augmented power. The solution was considered converged when the change in power was less than $0.5 \%$ from the extrapolated mean over a few hundred time steps. Evaluating the mean power from $t=0$ to time step $t=t_{i}$, defined in Equation 3.11 as

$$
\overline{P_{i}}=\frac{\sum_{t=0}^{t=t_{i}} P_{i}}{N_{i}}
$$

The assumed 1s time step from the previous time step study was used to conduct transient mesh independence study of the previous scaled meshes. Figure 3.16 shows convergence values of the mean augmented power ratio of the four meshes with respect to node density.

Deviations of the resultant grid-time convergence study are shown in Table 3.5. It can be seen that mesh 4 deviated from the extrapolated result by $0.66 \%$.

For case tested for mesh and time convergence, no taper was applied to the BAWT but was analyzed with a wind incidence angle of $0^{\circ}$. It is assumed smaller vortex will be shed in tapered cases, thus the finer mesh should be used to capture the smaller vortex scales. Modeling the smaller turbulent scales more accurately will lead to better pressure fluctuation results aft of the turbine to better predict augmented power at the rotor. Therefore mesh 4 will be used for the purpose of this study. 


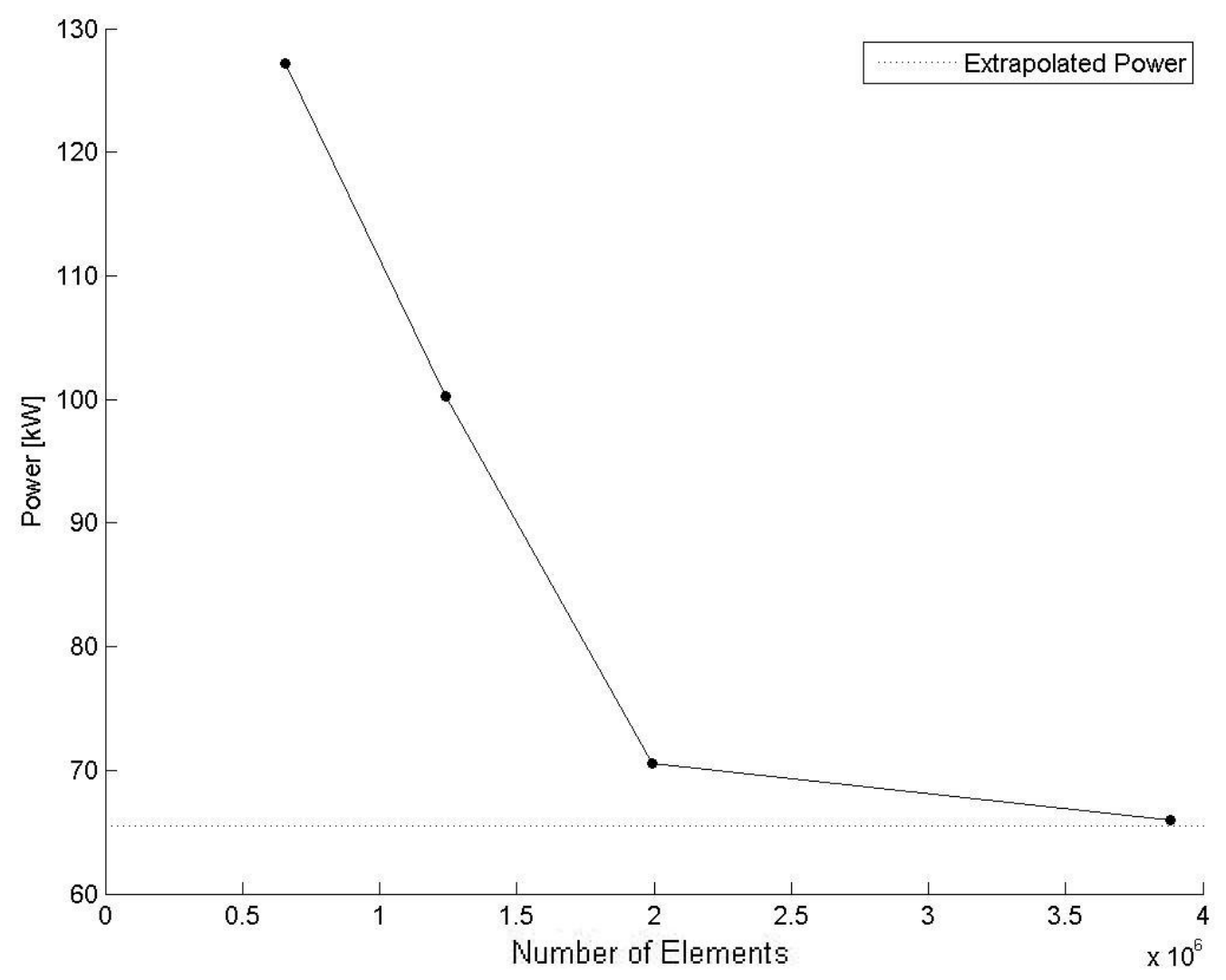

Figure 3.16: Grid convergence at $t_{i}=1 \mathrm{~s}$

\begin{tabular}{c|r|c} 
Mesh & Elements & $\begin{array}{c}\text { Deviations from } \\
\text { Extrapolated Result }\end{array}$ \\
\hline 1 & 656,637 & $94.0 \%$ \\
2 & $1,241,258$ & $53.0 \%$ \\
3 & $1,994,056$ & $7.7 \%$ \\
4 & $3,883,171$ & $0.7 \%$
\end{tabular}

Table 3.5: Steady state grid convergence 


\section{Chapter 4}

\section{Methodology}

This section contains discussions of the approaches to the conditions for the solution boundaries, simulation parameters and the result criteria. Specified solution parameters for turbulent modeling were used to simulate the von Karman street. The analysis of power at the Turbine was used to determine solution convergence of the mean transient to a specified accuracy.

\subsection{Boundary Conditions}

Atmospheric boundary layer (ABL). ABL is the naturally occurring velocity profile developed when air passes over Earths terrain. Viscous forces become dominant near the terrain where shear forces resist fluid momentum until zero velocity is reached at the surface. Zero velocity at the surface is considered a no slip condition.

A commonly used model to define the velocity profile of ABL is the logarithmic law (Log-Law). A Log-Law defining the boundary layer over a flat plate was derived by Prandtl was also found to be valid to define the ABL near the Earths surface [15. Velocity profile from a referenced height of $60 \mathrm{~m}$ is used

to calculate the velocity profile for any reference wind speed. ABL defined by Holmes [15] was simplified with defining a surface roughness, $z_{o}$ of $0.03 \mathrm{~m}$ and represented as Equation 4.119 .

$$
u_{(z)}=1.31 u_{p} \frac{\ln \left(\frac{z}{z_{o}}\right)}{\ln \left(\frac{60}{z_{o}}\right)}
$$

where $u_{p}$ is the potential wind speed $10 \mathrm{~m}$ above ground and $z$ is the elevation height.

The Log-Law profile was used with the velocity inlet condition to define the ABL normal to the Inlet boundary with a $10 \%$ turbulence intensity suggested by ANSYS when the inlet turbulent characteristics are unknown. Figure 4.1 shows the ABL velocity vectors and stream lines along a segment of the domain.

A gauge pressure of $0 \mathrm{~Pa}$ far field was chosen for pressure at the Outlet condition.

Symmetry conditions were imposed on the Sides and Top boundaries of the domain to artificially 


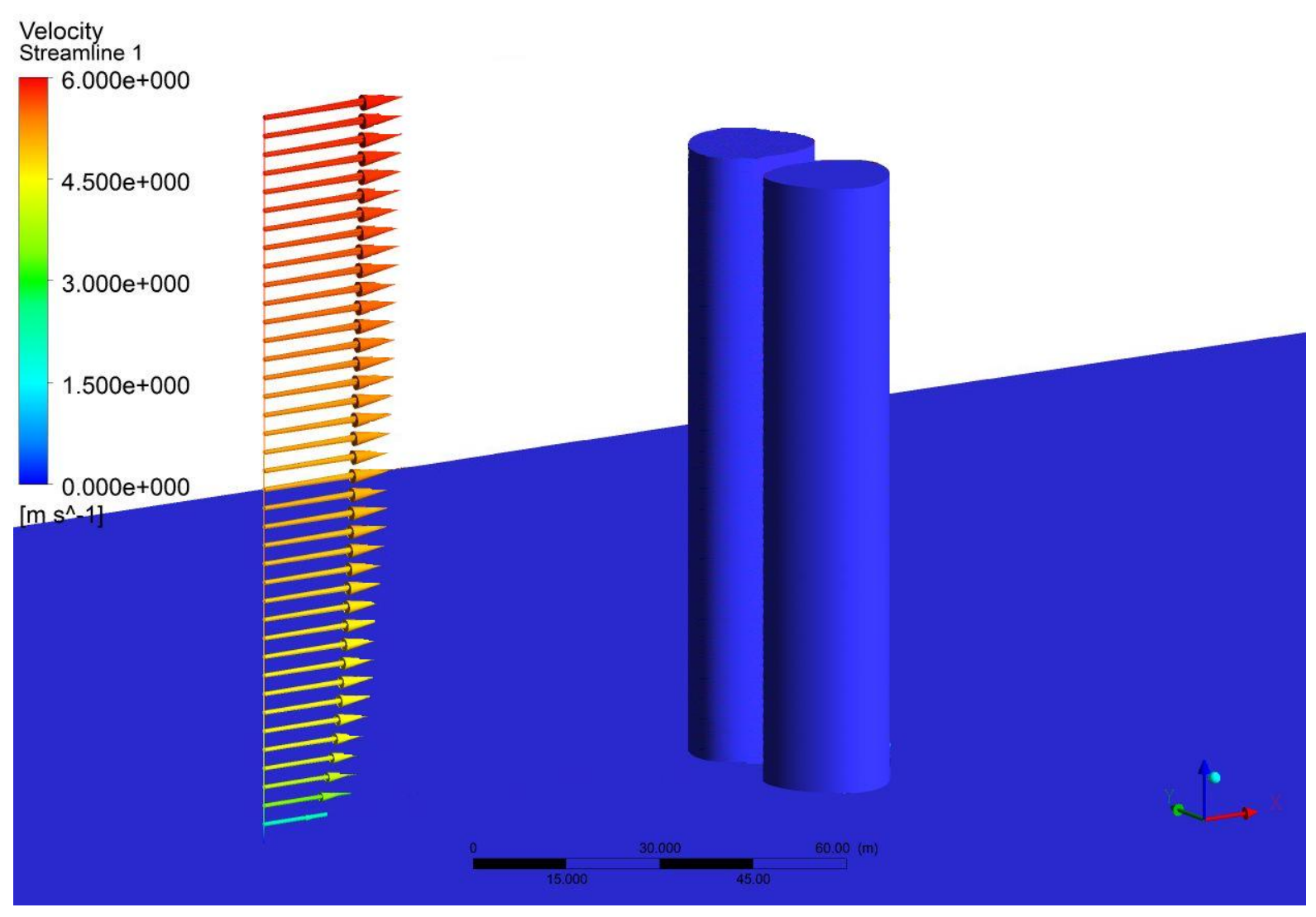

Figure 4.1: Log-Law velocity profile of ABL 
simulate a greater domain space. Flow variables are allowed to develop along these symmetry boundaries where the normal gradients of the flow variables are equated to zero. Normal gradients however are not zero at the Walls and Ground boundaries. A no-slip condition is used for the two boundaries where the surface roughness $z_{o}$ of $0.03 \mathrm{~m}$ is defined for the Ground to simulate terrain. The Walls are assumed to be smooth [22] thus zero surface roughness was used.

The Turbine boundary was defined with a porous jump condition where a pressure drop was applied to simulate the presence of a turbine. A simplified Navier-Stokes equation for momentum derived in Appendix $\mathrm{C}$ was used to define the pressure drop across the Turbine in Equation C.2 as

$$
\Delta P=-C_{2} \frac{1}{2} \rho u^{2} \Delta m
$$

where $\rho$ is the density of the fluid, $C_{2}$ is the momentum loss term corresponding to the pressure loss coefficient defined by Hans Hamm as 0.4 [14, $u$ is the velocity magnitude normal to the cell face, and $\Delta m$ is the thickness of the porous jump.

\subsection{Solution Parameters}

The solver used for this CFD analysis is FLUENT (ANSYS Inc.). This solver was chosen because of its reputation for solving both high and low Reynolds flows and around bluff bodies and the ability to obtain good boundary layer flow characteristics in all flow regimes. A density-based implicit solver was used where only Navier-Stokes equations are solved simultaneously using algebraic multi-grid (AMG) solver. A second-order upwind scheme recommended by ANSYS for accuracy in solving the discretized solution domain using the finite volume method was used.

The shear stress transport (SST) $k-\omega$ model was chosen over traditional $k-\epsilon$ model for its accuracy in solving adverse pressure gradients induced by wall bounded turbulent structures [2]. Mentor developed the SST which incorporates the qualities of models $k-\epsilon$ and $\mathrm{k}-\omega$ by using a blend factor to activate the $k-\omega$ to calculate shear stress at low Reynolds number within the flow boundary layers and at far field where negligible shear stresses occur the $\mathrm{k}-\epsilon$ model is activated.

\subsection{Solution Criteria}

The power of the wind at the Turbine boundary was used to measure mean transient conditions and is derived in Equation 4.3

$$
\overline{P_{i}}=\frac{\sum_{t=0}^{t=t_{i}} P_{i}}{N_{i}}
$$

The solution is said to be completed when consistent mean transient conditions are obtained in the simulations. The solution was determined to be found if augmented power remains to within a $0.5 \%$ 
deviation from the extrapolated mean of the augmented power for several hundred seconds.

The extrapolated mean augmented power was calculated using built in Richardson extrapolation function within Matlab to calculate the convergence value of mean augmented power. Plots of the solution convergence is shown in Figure 4.2 and Figure 4.3 is the associated error with respect to the converged value.

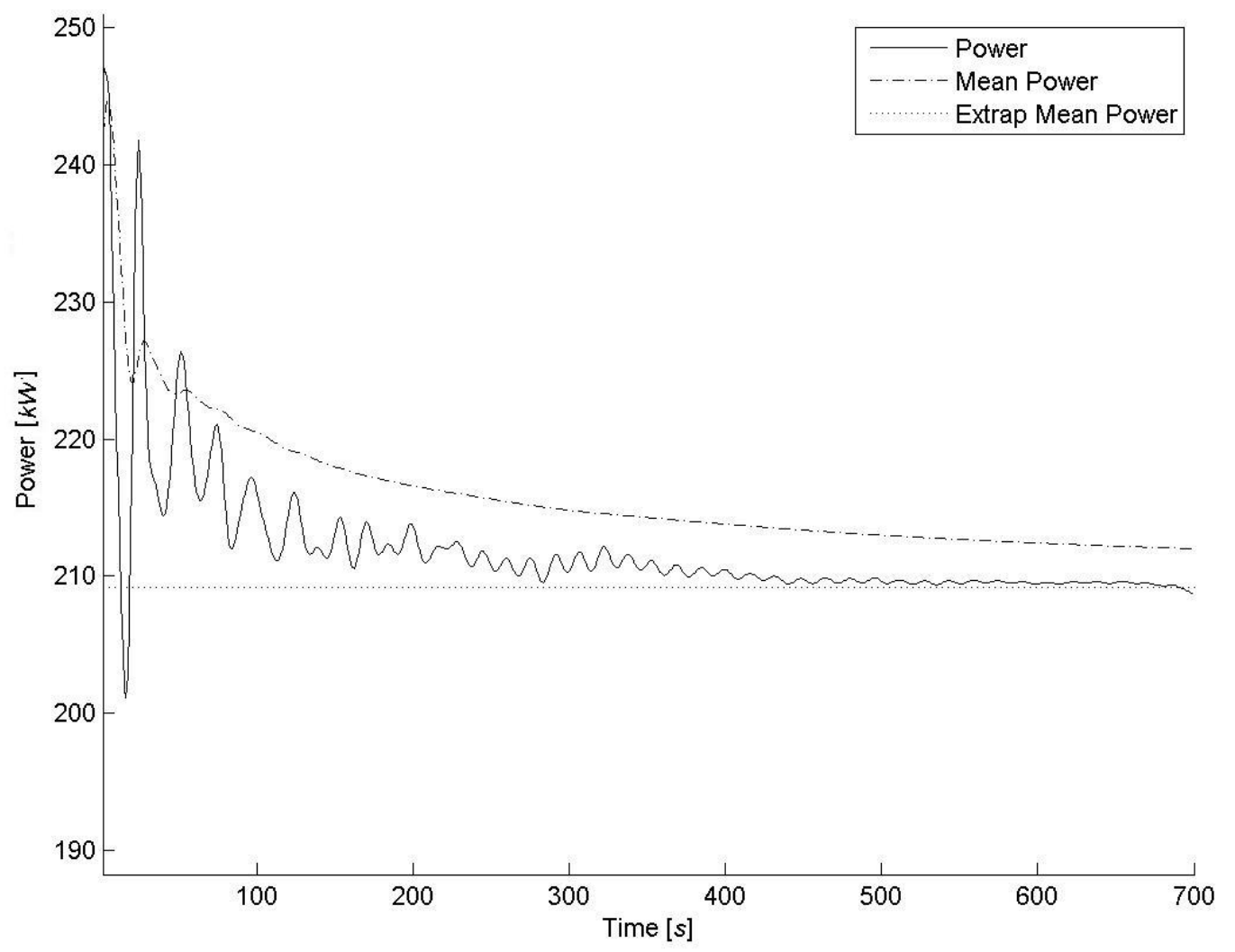

Figure 4.2: Mean time power covergence study $\left(R_{t}=0.33, R_{x}=0, R_{y}=1, \alpha=0^{\circ}\right)$

Various BAWT configurations produced oscillations of the augmented power at high incidence angles and wide spread. Pressure fluctuations formed from vortex shedding occurring at the Turbine boundary. An oscillating solution was considered converged when the augmented power oscillations maintain consistent amplitude for several hundred seconds as shown in Figure 4.4. 


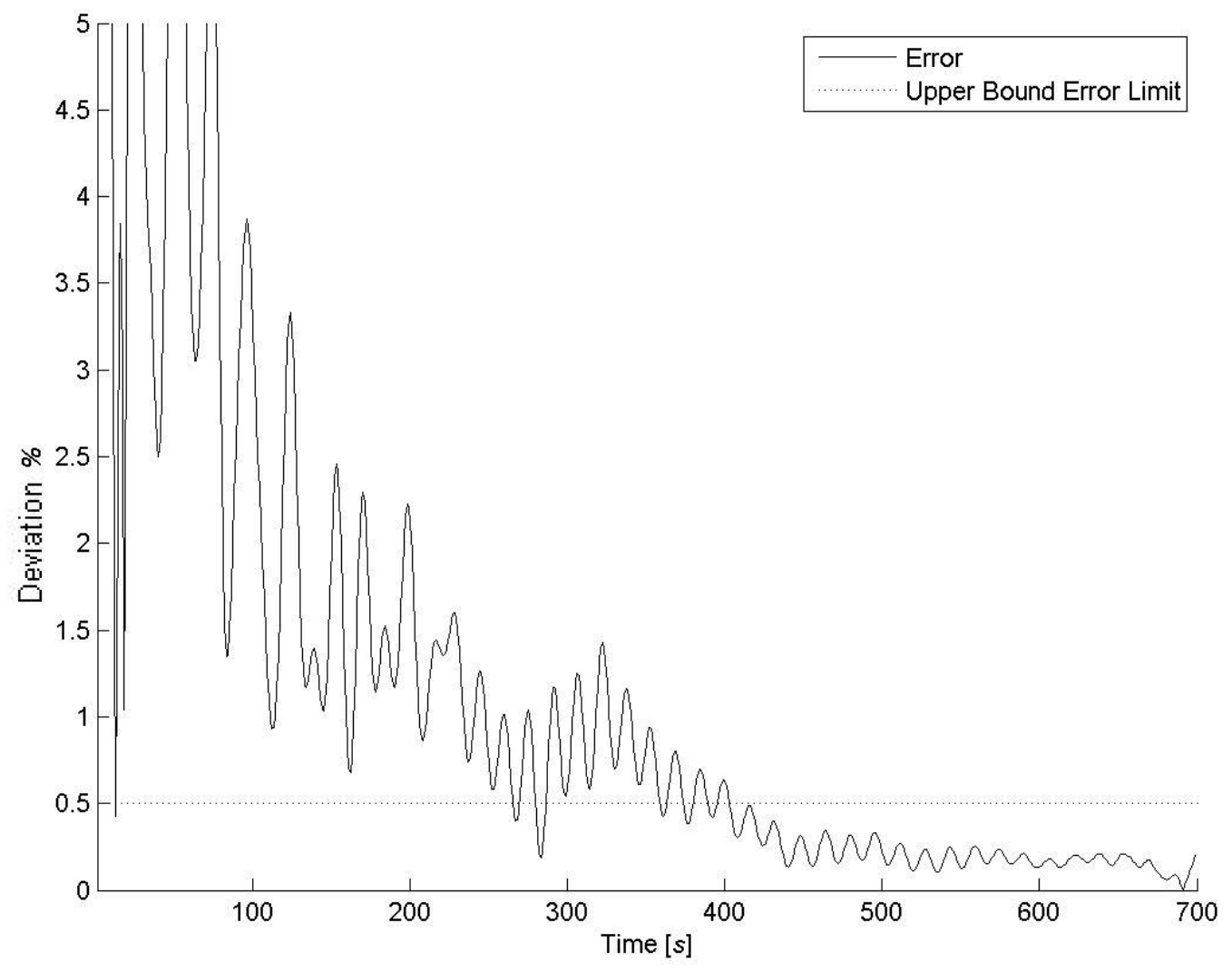

Figure 4.3: Error covergence study $\left(R_{t}=0.33, R_{x}=0, R_{y}=1, \alpha=0^{\circ}\right)$ 


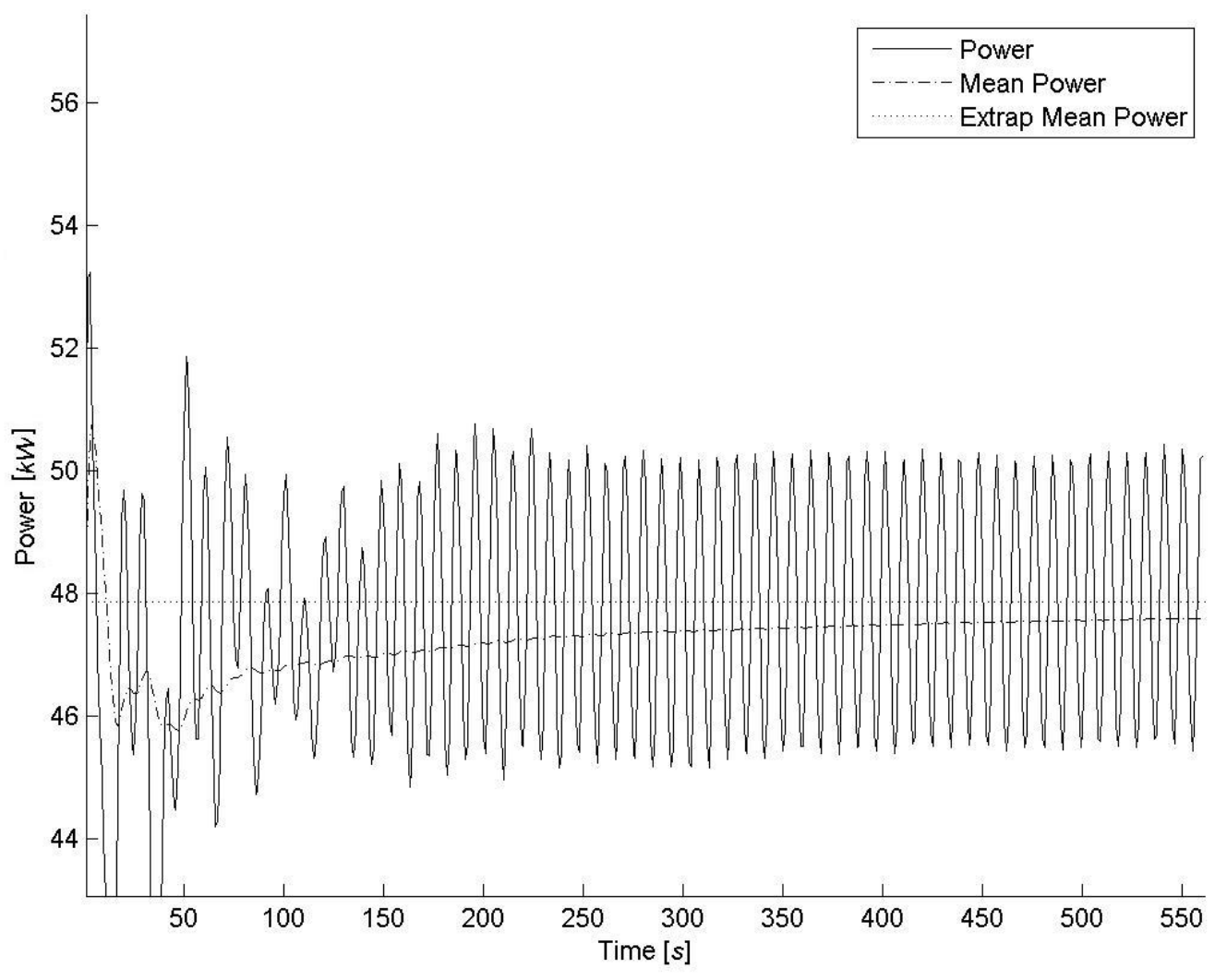

Figure 4.4: Mean time power covergence study $\left(R_{t}=0.33, R_{x}=0.5, R_{y}=1, \alpha=60^{\circ}\right)$ 


\subsection{Augmented Power}

Power is the rate of energy of a mass flow of air passing through a rotor swept area is defined as,

$$
\frac{\delta E}{\delta t}=P=\iint_{A} \epsilon \rho(V \cdot n) \delta A
$$

Where $n$, is the normal vector of the sweeping area and $\epsilon$ is the energy per unit mass,

$$
\epsilon=u+\frac{1}{2} V^{2}+g z
$$

Simplifications are made by neglecting internal energy and the hydrostatic terms. Due to the incompressibility of the fluid and lack of heat transfer the internal energy is constant, the fluid is gravitational the potential energy remains constant thus they can be negated reducing the equation to the kinetic energy of the fluid.

$$
\epsilon=\frac{1}{2} V^{2}
$$

Substituting equation (4.6) into the rate of energy equation (4.4) to give energy flux as

$$
P=\frac{1}{2} \rho \iint_{A} V^{2}(V \cdot n) \delta A
$$

The density, $\rho$ remains constant and can be taken outside of the integral and $\partial A$ is the Turbine area. Turbine area is as function of building spread factor written as Equation 4.8

$$
A=y(z) z
$$

The change in area is then found as

$$
\partial A=z \partial y+y(z) \partial z
$$

The first term of the 4.9 is dropped because the Turbine only varies in the $y$-direction. Thus change in Turbine area becomes

$$
\partial A=y(z) \partial z
$$

Equation 4.10 can be substituted back into Equation 4.7 and simplifications are made 


$$
\begin{aligned}
P & =\frac{1}{2} \rho \iint_{A} V^{2}(V \cdot n) \delta A \\
P & =\frac{1}{2} \rho \int_{10}^{100} V(z)^{3} y(z) \partial z
\end{aligned}
$$

The integration in Equation 4.12 is calculated from height of $10 \mathrm{~m}$ to $100 \mathrm{~m}$ where the Turbine boundary is situated. 


\section{Chapter 5}

\section{Results}

An analysis of the data obtained from this study is synthesised and presented in various sections to illustrate of the purpose of this study. ANSYS FLUENT software was used to perform transient simulations of 76 BAWT cases where their wind augmentation performance was measured with respect to four wind incidence angles. The BAWT performances were measured by various criteria of augmentation of both velocity and power, and the ratio of augmented power to unaugmented power. Also along with analysing their power ratings using three different wind regimes with equivalent frequencies, power

rating was obtained from the sum of the product of the augmented power and the frequency of the wind incidence angle.

Figures 5.15 .2 shows all data relevant to the results sections. Only BAWT configuration with $R_{x}$ values at 0.5 are presented. This was done because change in results varied minutely with the change in $R_{x}$ for all cases.

\subsection{Velocity Augmentation}

One of the purposes of this study was to determine the velocity augmentation factor the BAWT configurations have on the imposing wind. The augmented velocity is the average normal velocity at the Turbine boundary. The unaugmented wind speed was taken as the average normal velocity of the ABL through Turbine boundary. Augmentation factors were calculated by normalizing the augmented velocity with the unaugmented velocity. The BAWT velocity augmentation factors were obtained for each of the various wind incidence angles and are shown in Figure 5.3

It can be seen from Figure 5.3 that augmentation factors for all BAWT configurations are at their highest at zero wind incidence angle and a gradual decrease occurs with increasing angles. Looking at the results for zero wind incidence angles, it is noted there the highest value of 1.3 is obtained for the case with no taper, $R_{t}$ is 0 . Velocity augmentation factors are shown to decrease by $1.5 \%$ and $4.6 \%$ with the increasing in taper from 0.33 and 0.66 respectively while and keeping an equivalent turbine capture area with a spread factor of 0 . 


\begin{tabular}{|c|c|c|c|c|c|c|c|c|c|c|c|}
\hline Rt & Ry & $\mathbf{R x}$ & WIA & $\begin{array}{l}\text { Vabl } \\
{[\mathrm{m} / \mathrm{s}]}\end{array}$ & $\mathrm{V} 2[\mathrm{~m} / \mathrm{s}]$ & v2/Vabl & $\begin{array}{l}\text { Palb } \\
{[k W]}\end{array}$ & $P[k W]$ & P/Pabl & $\begin{array}{c}\text { Area } \\
{\left[\mathrm{m}^{\wedge} 2\right]}\end{array}$ & $\begin{array}{c}\text { Density } \\
{\left[\mathrm{kW} / \mathrm{m}^{\wedge} 2\right]}\end{array}$ \\
\hline \multirow{4}{*}{0} & \multirow{4}{*}{0} & \multirow{4}{*}{0} & 0 & 5.28 & 6.85 & 1.30 & 49.0 & 106.2 & 2.17 & 540 & 0.197 \\
\hline & & & 20 & 5.28 & 6.81 & 1.29 & 49.0 & 103.1 & 2.11 & 540 & 0.191 \\
\hline & & & 40 & 5.28 & 6.03 & 1.14 & 49.0 & 81.4 & 1.66 & 540 & 0.151 \\
\hline & & & 60 & 5.28 & 5.59 & 1.06 & 49.0 & 57.5 & 1.17 & 540 & 0.106 \\
\hline \multirow{36}{*}{0.33} & \multirow{12}{*}{0} & \multirow{4}{*}{0} & 0 & 5.28 & 6.56 & 1.24 & 49.0 & 95.4 & 1.95 & 540 & 0.177 \\
\hline & & & 20 & 5.28 & 6.54 & 1.24 & 49.0 & 93.3 & 1.90 & 540 & 0.173 \\
\hline & & & 40 & 5.28 & 6.00 & 1.14 & 49.0 & 73.1 & 1.49 & 540 & 0.135 \\
\hline & & & 60 & 5.28 & 4.76 & 0.90 & 49.0 & 45.5 & 0.93 & 540 & 0.084 \\
\hline & & \multirow{4}{*}{0.5} & 0 & 5.28 & 6.77 & 1.28 & 49.0 & 100.3 & 2.05 & 540 & 0.186 \\
\hline & & & 20 & 5.28 & 6.59 & 1.25 & 49.0 & 92.3 & 1.89 & 540 & 0.171 \\
\hline & & & 40 & 5.28 & 5.94 & 1.13 & 49.0 & 71.5 & 1.46 & 540 & 0.132 \\
\hline & & & 60 & 5.28 & 4.98 & 0.94 & 49.0 & 47.3 & 0.97 & 540 & 0.088 \\
\hline & & \multirow{4}{*}{1} & 0 & 5.28 & 6.68 & 1.27 & 49.0 & 98.3 & 2.01 & 540 & 0.182 \\
\hline & & & 20 & 5.28 & 6.52 & 1.24 & 49.0 & 92.5 & 1.89 & 540 & 0.171 \\
\hline & & & 40 & 5.28 & 6.00 & 1.14 & 49.0 & 70.8 & 1.45 & 540 & 0.131 \\
\hline & & & 60 & 5.28 & 5.26 & 1.00 & 49.0 & 50.9 & 1.04 & 540 & 0.094 \\
\hline & \multirow{12}{*}{0.5} & \multirow{4}{*}{0} & 0 & 5.34 & 5.94 & 1.11 & 92.3 & 159.0 & 1.72 & 986.8 & 0.161 \\
\hline & & & 20 & 5.34 & 6.20 & 1.16 & 92.3 & 143.6 & 1.56 & 986.8 & 0.145 \\
\hline & & & 40 & 5.34 & 5.59 & 1.05 & 92.3 & 106.9 & 1.16 & 986.8 & 0.108 \\
\hline & & & 60 & 5.34 & 3.71 & 0.69 & 92.3 & 48.8 & 0.53 & 986.8 & 0.049 \\
\hline & & & 0 & 5.34 & 6.41 & 1.20 & 92.2 & 158.9 & 1.72 & 986.8 & 0.161 \\
\hline & & 05 & 20 & 5.34 & 6.19 & 1.16 & 92.2 & 143.1 & 1.55 & 986.8 & 0.145 \\
\hline & & & 40 & 5.34 & 5.32 & 1.00 & 92.2 & 99.6 & 1.08 & 986.8 & 0.101 \\
\hline & & & 60 & 5.34 & 3.54 & 0.66 & 92.2 & 52.4 & 0.57 & 986.8 & 0.053 \\
\hline & & & 0 & 5.34 & 6.38 & 1.19 & 92.3 & 157.3 & 1.70 & 986.8 & 0.159 \\
\hline & & 1 & 20 & 5.34 & 6.08 & 1.14 & 92.3 & 138.5 & 1.50 & 986.8 & 0.140 \\
\hline & & 1 & 40 & 5.34 & 5.40 & 1.01 & 92.3 & 107.8 & 1.17 & 986.8 & 0.109 \\
\hline & & & 60 & 5.34 & 2.98 & 0.56 & 92.3 & 33.8 & 0.37 & 986.8 & 0.034 \\
\hline & & & 0 & 5.36 & 6.20 & 1.16 & 135.6 & 209.2 & 1.54 & 1433.5 & 0.146 \\
\hline & & 0 & 20 & 5.36 & 5.89 & 1.10 & 135.6 & 179.5 & 1.32 & 1433.5 & 0.125 \\
\hline & & 0 & 40 & 5.36 & 5.04 & 0.94 & 135.6 & 126.2 & 0.93 & 1433.5 & 0.088 \\
\hline & & & 60 & 5.36 & 3.10 & 0.58 & 135.6 & 47.5 & 0.35 & 1433.5 & 0.033 \\
\hline & & & 0 & 5.36 & 6.18 & 1.15 & 135.5 & 208.8 & 1.54 & 1433.5 & 0.146 \\
\hline & 1 & 0.5 & 20 & 5.36 & 5.92 & 1.10 & 135.5 & 184.1 & 1.36 & 1433.5 & 0.128 \\
\hline & 1 & 0.5 & 40 & 5.36 & 5.26 & 0.98 & 135.5 & 129.0 & 0.95 & 1433.5 & 0.090 \\
\hline & & & 60 & 5.36 & 3.32 & 0.62 & 135.5 & 47.9 & 0.35 & 1433.5 & 0.033 \\
\hline & & & 0 & 5.36 & 6.22 & 1.16 & 135.6 & 210.5 & 1.55 & 1433.5 & 0.147 \\
\hline & & 1 & 20 & 5.36 & 5.97 & 1.11 & 135.6 & 186.3 & 1.37 & 1433.5 & 0.130 \\
\hline & & 1 & 40 & 5.36 & 5.25 & 0.98 & 135.6 & 128.8 & 0.95 & 1433.5 & 0.090 \\
\hline & & & 60 & 5.36 & 3.19 & 0.59 & 135.6 & 44.7 & 0.33 & 1433.5 & 0.031 \\
\hline
\end{tabular}

Figure 5.1: Results 


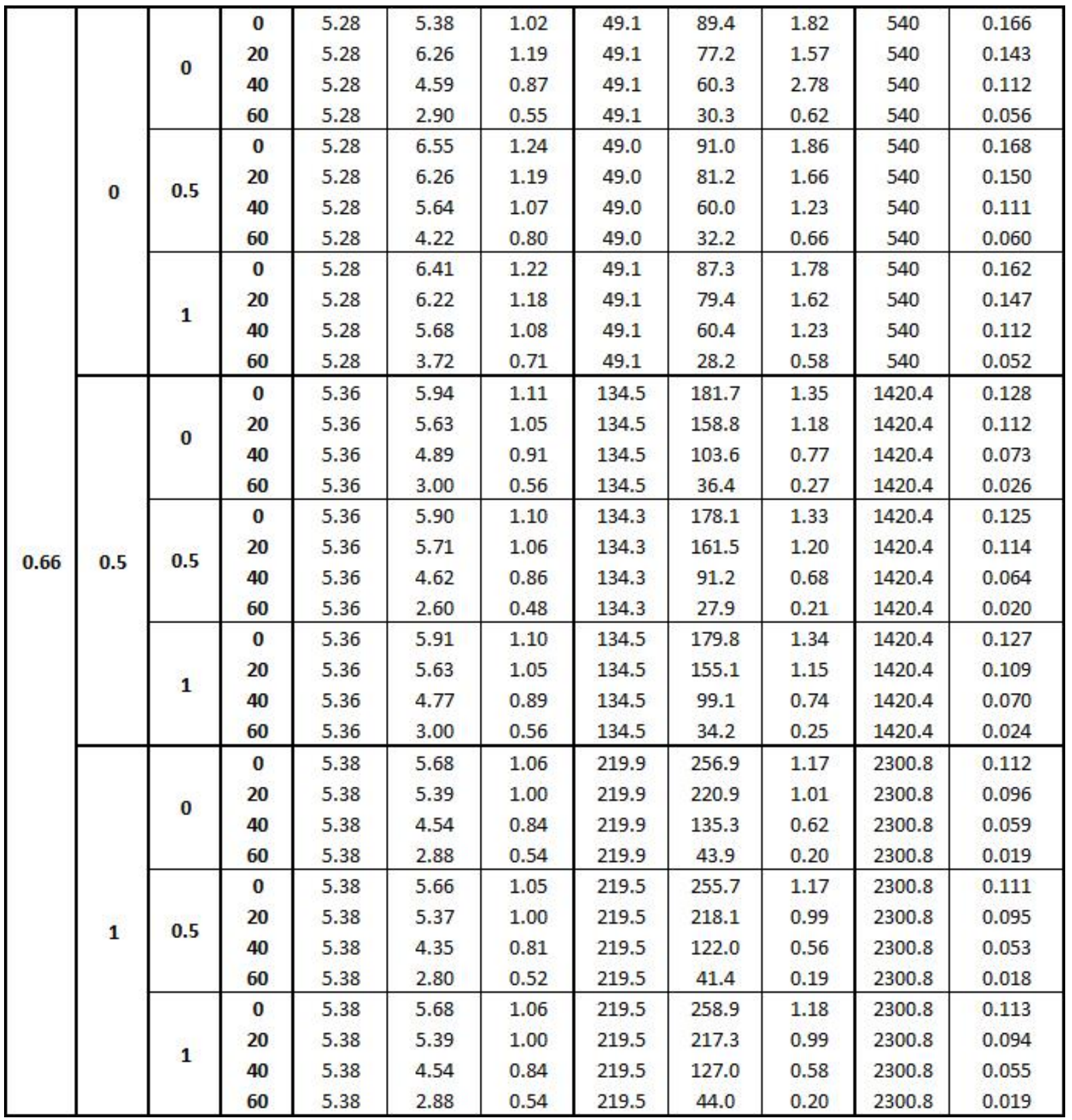

Figure 5.2: Results continued 


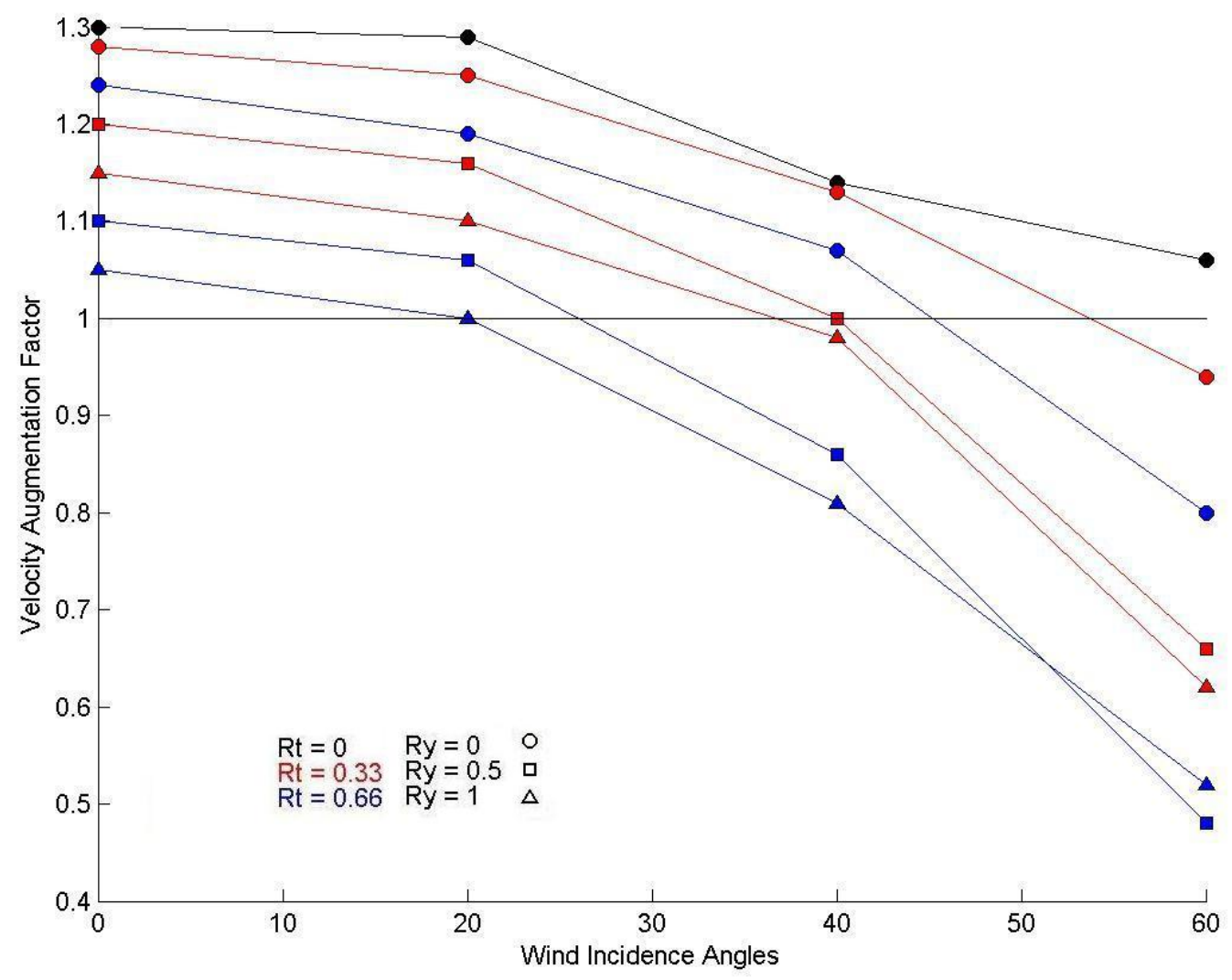

Figure 5.3: Velocity augmentation factors 
The spread factor $R_{y}$ is shown to have a negative effect on velocity augmentation factors for all cases. At zero incidence angle and using spread factor of 1 and 0.5 for cases of taper $R_{t} 0.33$ and 0.66 respectively, augmentation factors decreased from their 0 spread configuration by $10.2 \%$ and $11.3 \%$ and respectively. These results correlate to the law of continuity well, which states the incompressible flow must increase velocity through the BAWT gap region to maintain mass flow conservation. The velocity is also related to the pressure through the system where greater pressure differentials create higher velocities.

Figure 5.3 also illustrates the angles at which the BAWT configurations produce no velocity augmentation. Augmentation factors below 1 depict a decrease in performance values comparative to the free stream ABL. The BAWT configuration with taper 0.66 and spread of 1 is shown to prove no augmentation for incidence at $20^{\circ}$ and poor augmentation for angles greater than $20^{\circ}$. Positive augmentation remains for at higher angles with decrease in spread factor, and the decrease in taper. The BAWT with 0 taper and 0 spread factors maintained to generate the highest augmentation factor for all incidence angles.

\subsection{Augmented Power}

This section analyses the augmented power delivered to the turbine capture area for all BAWT configurations with a comparison to the power in the ABL at the Turbine boundary.

\subsubsection{Total Power}

The total power within the flow at the Turbine boundary was obtained from the integral power developed normal to the Turbine boundary. Total power was obtained for each BAWT and was graphed in correlation to the four wind incidence angles shown in Figure 5.4

Figure 5.4 shows there is a clear case when taper is 0.66 with spread factor of 1 generates the greatest energy of $255.7 \mathrm{~kW}$ at the Turbine at $0^{\circ}$ wind incidence. It is interesting to note from the previous section, this case provided the least velocity augmentation but outperforms in power generation. Moreover, the tapered cases with 0 spread factors which produce the highest wind augmentation factors result in generating the least power. This suggests the study of wind power generation is sensitive to changes in the capture area of turbines.

For the cases with spread factor of 0 with equivalent capture areas of $540 \mathrm{~m}^{2}$, the total augmented power of the three cases for taper of $0,0.33$ and 0.66 are $106.2 \mathrm{~kW}, 100.3 \mathrm{~kW}$, and $91 \mathrm{~kW}$ respectively with a difference of $10.6 \%-11.7 \%$. Changes in capture area produce wide degree of augmented power for the BAWT configurations. When spread factor was increased from 0 to 0.5 , the Turbine boundary area increased $82.7 \%$ and $165.5 \%$ for tapers 0.33 and 0.66 respectively. This increased area augments the total power by $108.2 \%$ and $95.8 \%$ for the tapered cases of 0.33 and 0.66 respectively.

The configuration with the lowest power production at $0^{\circ}$ wind incidence angle is taper ratio of 0.66 with spread factor of 0 . This resulted from the lack of velocity augmentation through the BAWT where the flow does not accelerate comparably to the cases with increases in taper ratio and 0 spread factor. 


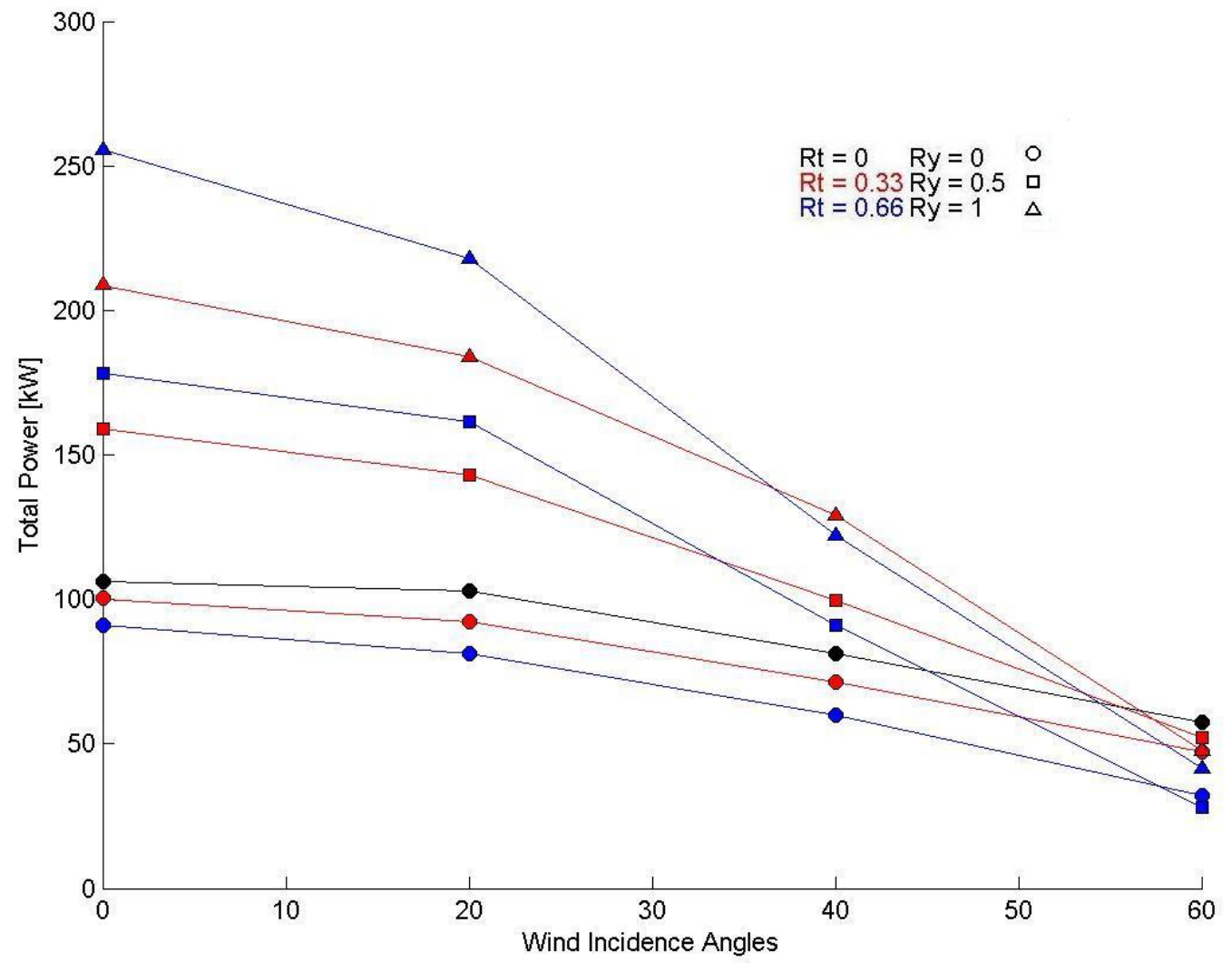

Figure 5.4: Total Power 
Looking at Figure 5.4 from the best configuration to operate at $60^{\circ}$ is the configuration with 0 taper. It outperforms configurations with taper at maximum spread factor of 1 by $20.1 \%$ and $38.8 \%$ for tapers of 0.33 and 0.66 respectively. Power values decrease with increasing wind incidence angle. This is an adequate result with correlations to the venturi effect with respect to incidence where venturi factor decreased as a result of the lack of low pressures aft of the BAWT system.

The case where taper is 0.33 at max spread factor of 1 surpasses the total power of cases with taper 0.66 and 0 is at a wind incidence angle of $30^{\circ}-40^{\circ}$. The difference in total power is $6.75 \mathrm{~kW}$, a difference of $5.6 \%$ and $15.6 \%$ at $40^{\circ}$ and $60^{\circ}$ wind incidence angles respectively. The two cases are surpassed by case of 0 taper between $50^{\circ}-60^{\circ}$. The measured powers at wind incidence angles of $60^{\circ}$ are; $41.4 \mathrm{~kW}$, $47.9 \mathrm{~kW}$, and $57.5 \mathrm{~kW}$, with a difference of $38.8 \%$ and $20 \%$ with respect to the cases when taper of 0.33 and 0.66 , both spread factors at 1 respectively. The critical factor controlling power production is the building spread which maximize turbine capture area, and higher wind augmentation factor plays a minimal role in the overall total power of a BAWT.

\subsubsection{Power Augmentation Factor}

Power augmentation factor determines how much power augmentation the specific BAWT configurations have in comparision to the ABL. The power augmentation factor is the ratio of the measured augmented total power to the total power from an unaugmented ABL. Power augmentation factors were measured for each BAWT configuration at each of the wind incidence angles and are plotted in Figure 5.5 .

An average drop in power augmentation factor of 0.5 occurs when spread increases from 0 to 0.5 and an average drop of 0.2 occurs from 0.5 to 1 . Power augmentation factor of 1 occurs just below $50^{\circ}$ for spread factor of 0 and occurs at $30^{\circ}$ and $20^{\circ}$ with $R y=0.5$ and $R y=1$ respectively. A factor of 1 shows when a BAWT system produces no increase in total power compared to the power available in the ABL.

\subsection{Power Rating}

This study looked into defining the power rating of BAWT configurations varying wind regimes. Power rating is defined as the sum products of the augmented power and corresponding wind frequencies of the wind regime. Wind regimes are frequency of the measured wind directions based on the statistical averaged for a particular location.

Frequency of wind regimes are location dependent and any specified frequencies would be arbitrary. For this study three even frequency regime distributions are analysed where their frequencies of the wind incidence angles are equivalent and the regimes sum total of the frequencies equate $100 \%$. Also a case study with a wind regime based on referenced statistical yearly averages at a referenced location is studied for a more realistic analysis of BAWT power rating. 


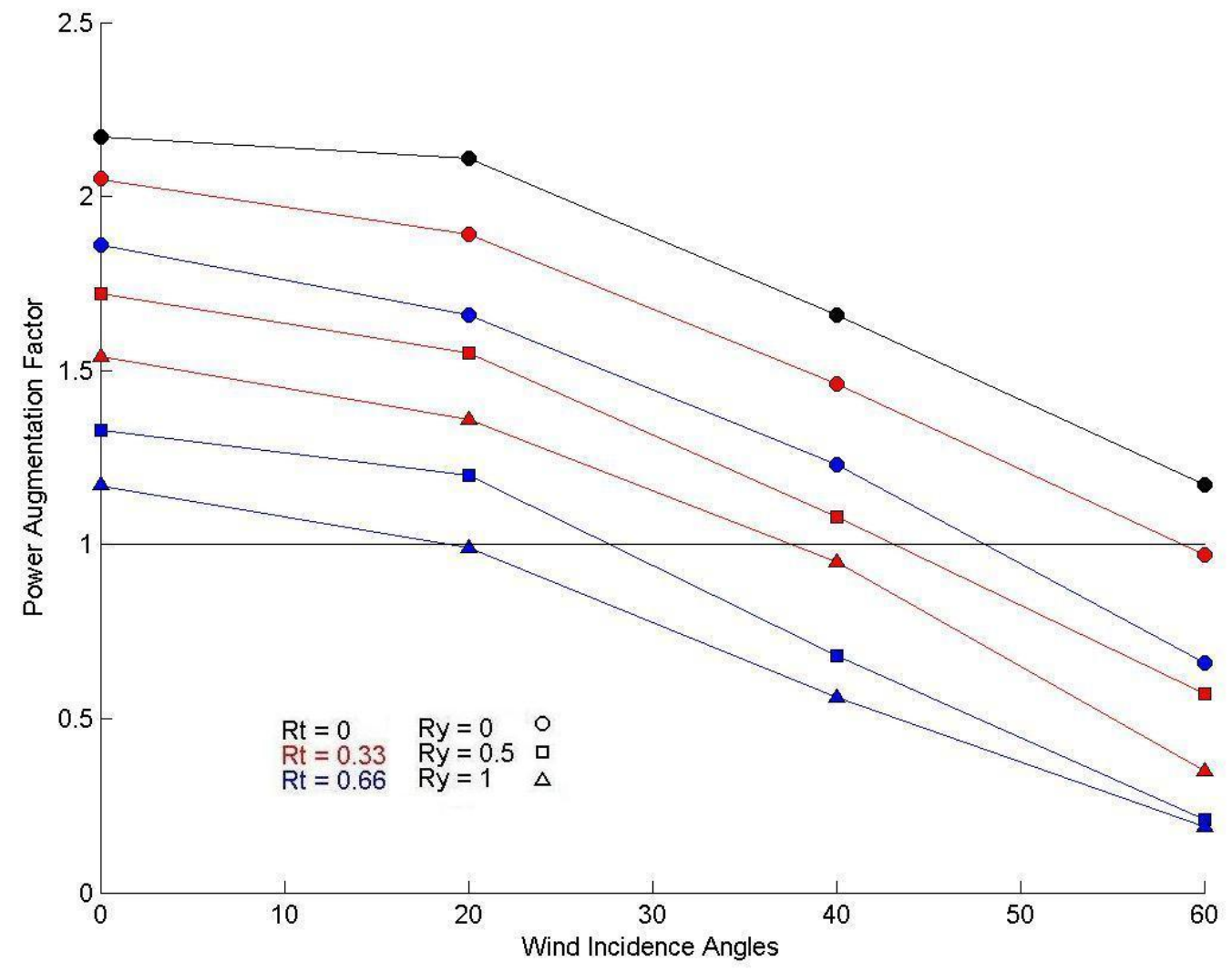

Figure 5.5: Power augmentation factors 


\begin{tabular}{r|c|c|c|c|c|c|c} 
Wind Regime & \multicolumn{7}{|c}{ Frequencies } \\
\hline High & $14.3 \%$ & $14.3 \%$ & $14.3 \%$ & $14.3 \%$ & $14.3 \%$ & $14.3 \%$ & $14.3 \%$ \\
Medium & - & $20 \%$ & $20 \%$ & $20 \%$ & $20 \%$ & $20 \%$ & - \\
Low & - & - & $33.3 \%$ & $33.3 \%$ & $33.3 \%$ & - & -
\end{tabular}

Table 5.1: Incidence angles and relative frequencies of wind regimes

\subsubsection{Even Frequency Regimes}

This section is sub divided into three sub sections each pertaining to the analysis of each wind regime given in Table 5.1. The power ratings are plotted with respect to their turbine capture area.

\section{High Regime}

The high wind regime incorporates all wind incidence angles tested ranging from $0^{\circ}-60^{\circ}$. Since the study contained a symmetry plain through the BAWT at $0^{\circ}$, the negative reflective angles are taken into account creating a wind azimuth of $\pm 60^{\circ}$. The frequencies of the seven incidence angle are assumed to be equal value of $14.3 \%$ to sum $100 \%$. The rated power for the BAWT system for a high wind regime is shown in Figure 5.6 .

Figure 5.6 depicts the power ratings for each BAWT configuration in the high regime with respect to the turbine capture area. Figure 5.6 shows when spread factor is constant at 0 , the power rating is highest for cases of 0 taper and produces a power rating of $84 \mathrm{~kW}$. The other ratings vary $12.9 \%$ and $38.2 \%$ for tapers of 0.33 and 0.66 respectively from the case of 0 taper.

It is interesting to note there are two cases of similar power ratings. The cases of tapered 0.33 and 0.5 spread factor is rated at $105.9 \mathrm{~kW}$ and case of 0.66 tapper and spread factor of 0.5 is rated at $108.5 \mathrm{~kW}$ with a difference of $2.4 \%$. The greater power rating developed by wind augmentation is counter balanced by a greater capture area.

For the case of 0.33 taper and spread factor of 1 the power rating is $22 \%$ higher than case of 0.66 taper at its maximum spread factor of 1 with equivalent Turbine areas. The velocity augmentation becomes the defining factor for energy capture rating.

\section{Medium Regime}

The medium wind regime incorporates the wind incidence angles ranging from $0^{\circ}-40^{\circ}$. Symmetry plane previously mentioned used at $0^{\circ}$ bisecting the BAWT generating a wind azimuth of $\pm 40^{\circ}$. Equal frequencies for each wind incidence angle are $20 \%$ summing to $100 \%$. Figure 5.7 depicts the power rating the BAWT configurations in the medium regime.

Equivalent trends to that of the high regime are produced for the medium regime were the results appear as scaled high regime results. For a constant spread factor of 0 of all tapered cases, the highest power rating of $95.1 \mathrm{~kW}$ is from the case with no taper, with an $11.3 \%$ and $29.6 \%$ difference in power ratings from cases when taper is 0.33 and 0.66 respectively. 


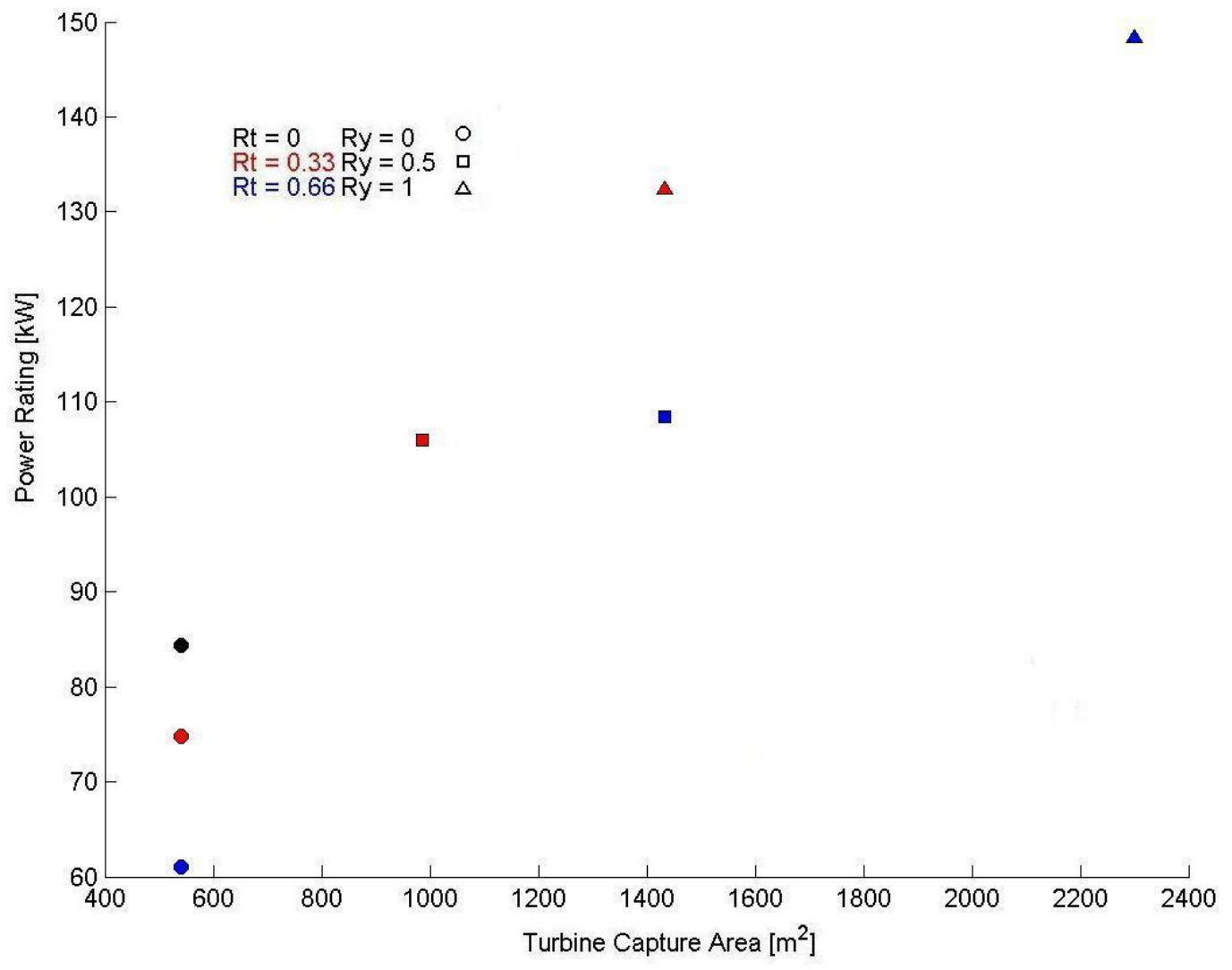

Figure 5.6: Power rating for high regime 


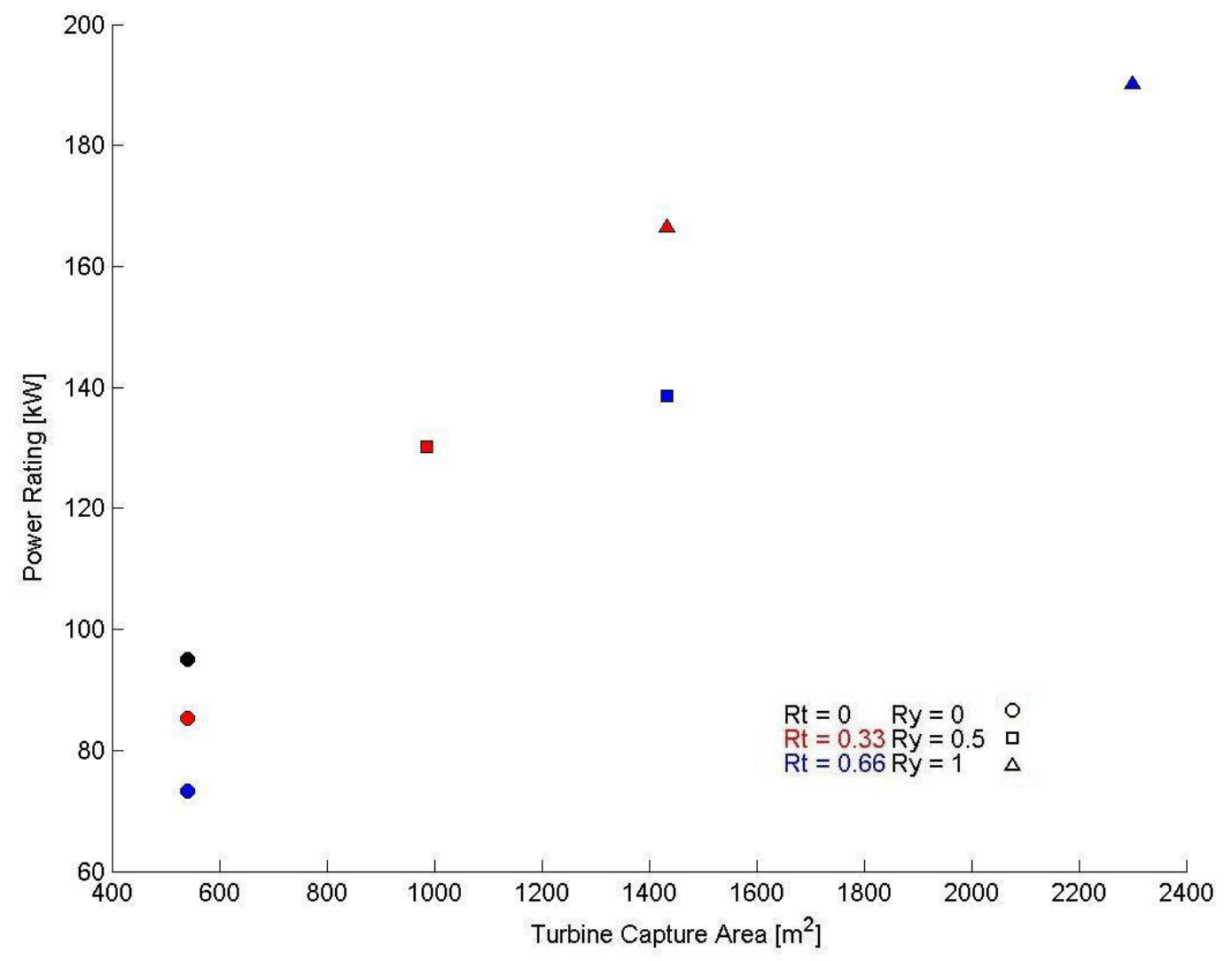

Figure 5.7: Power rating for medium regime 
The two same cases are in close proximity also within the medium regime. Their values of $130.3 \mathrm{~kW}$ and $138.8 \mathrm{~kW}$ for the cases of taper at 0.33 with spread factor of 0.5 and case of taper $0.66 \%$ with spread factory of 0.5 respectively. The difference of these values is $6.4 \%$. When the case of 0.33 taper has capture area equal to case with taper 0.66 , a difference of $20 \%$ is shown.

The highest energy captures obtained from all tapers were generated at their max spread factors where turbine capture area is maximized. These values are given in Table 5.3 were the differences compared to the case of 0 taper are $75.1 \%$ and $100 \%$ from tapers of 0.33 and 0.66 respectively.

\section{Low Regime}

The low wind regime incorporates the wind incidence angles ranging from $0^{\circ}-20^{\circ}$ with a symmetry plane at $0^{\circ}$ generates a wind azimuth of $\pm 20^{\circ}$. Equal frequencies of each wind incidence angle are $33.3 \%$ summing to $100 \%$. Figure 5.8 depicts the power rating of each BAWT configuration for a low regime.

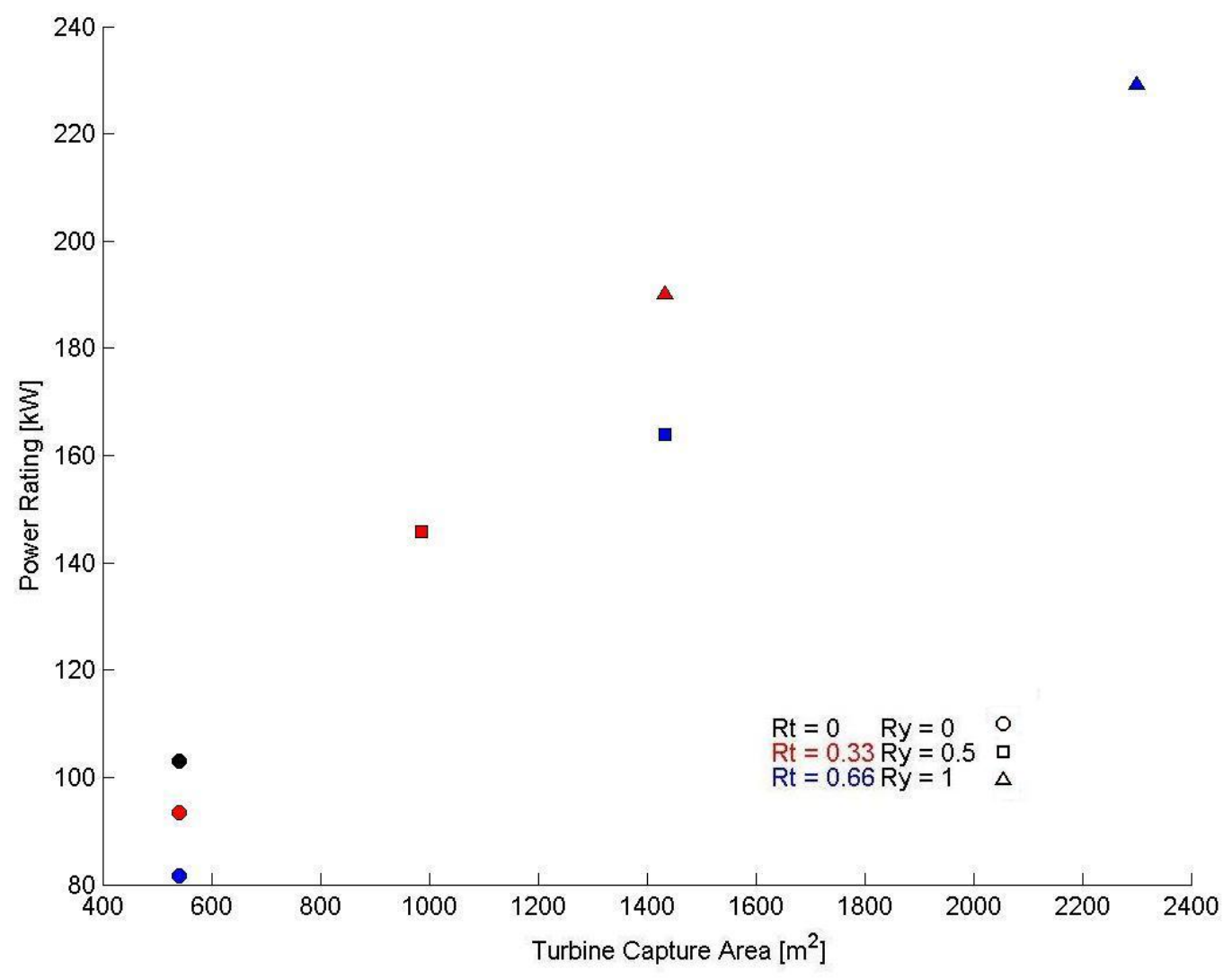

Figure 5.8: Power rating for low regime

Comparisons of the tapered cases with 0 spread factor generate differences of $10.2 \%$ and $22.8 \%$ for cases with taper 0.33 and 0.66 when compared to 0 taper of power rating of $103.1 \mathrm{~kW}$. There is a close 


\begin{tabular}{r|c|c|c|c|c|c|c} 
Incidence Angle & $-60^{\circ}$ & $-40^{\circ}$ & $-20^{\circ}$ & $0^{\circ}$ & $20^{\circ}$ & $40^{\circ}$ & $60^{\circ}$ \\
\hline Toronto Island Regime & $5.9 \%$ & $6.5 \%$ & $5.9 \%$ & $6.5 \%$ & $5.9 \%$ & $5 \%$ & $6.5 \%$
\end{tabular}

Table 5.2: Wind regime of Toronto Island

grouping of configurations that is noted within Figure 5.8. The values for of these configurations are shown in Table 5.3 .

The differences of cases of 0.33 taper with spread factor of 0.5 and 0.66 taper at spread factor 0.5 is $12.5 \%$. When the 0.33 taper case increases to spread factor of 1 , it then out performs the 0.66 taper with an equal turbine capture area by $20.5 \%$. Power rating is maximized for each taper case when maximum spread factor and turbine capture area is reached. The power rating increases $84.5 \%$ and $122.4 \%$ from tapered cases 0.33 and 0.66 with respect to the case of 0 taper.

\subsubsection{Wind Regime Case Study}

The previous power ratings made use of an even frequency distribution across the evaluated wind regimes which generates an over evaluates the power rating. A more realistic approach is taking in the section where measured values of yearly averaged wind characteristics are refferenced from the Toronto Island Airport, Toronto, Ontario. The wind regime's frequency was obtained by from a wind rose plot and used to calculate the power rating. Finally power ratings for all the BAWT configurations analysed are made and compared.

A wind rose plot is essential in determining the appropriate site for wind turbines and BAWT systems. It states three main characteristics of the wind engineers need to determine if a location is suitable in harvesting wind. The three characteristics are: speed, direction and frequency. A wind rose plot from the Toronto Island Airport was select for this study for its comparable ABL to the simulated ABL. Winds are coming off the water where the surface roughness is low and close to that of the simulated $0.03 \mathrm{~m}$ surface roughness. Figure 5.9 is the wind rose plot of the Toronto Island Airport.

It can be seen from 5.10 the dominant wind direction is of West South West direction. Thus the BAWT system was oriented to face into that direction and have the BAWT's plane of symmetry directed West South West. Figure 5.10 illustrates the orientation of the BAWT system and it's encompassing wind azimuth of $\pm 60^{\circ}$.

The sectors for the wind rose plot are sepparated by $22.5^{\circ}$ and it was assumed the sectors are close enough to the simulated wind sectors of $20^{\circ}$. The wind incidence angles and their frequencies are tabulated in Table 5.2 .

Similarly to the previous section, power rating was defined as the sum products of the augmented power and corresponding wind frequencies of the wind regime. It is noted the sum of the frequency for the regime within the BAWT azimuth totals $42.2 \%$, hence a lower power rating will incur. The power ratings for the BAWT configurations are presented in Figure 5.11.

Figure 5.11 depicts the power ratings for each BAWT configuration Toronto Island Airport wind regime with respect to the turbine capture area. Figure 5.11 shows when spread factor is constant at 
Toronto Island (TORONTOI)

Statistics based on observations taken between $2 / 2007-3 / 2011$ daily from 7 am to 7 pm local time.

Month of year
Dominant Wind dir.
Wind probability
$>=4$ Beaufort ( $\%$ )
Average
Wind speed
(Knots)
Average air temp. $\left({ }^{\circ} \mathrm{C}\right.$ )
Select month (Help)

select month (Help)

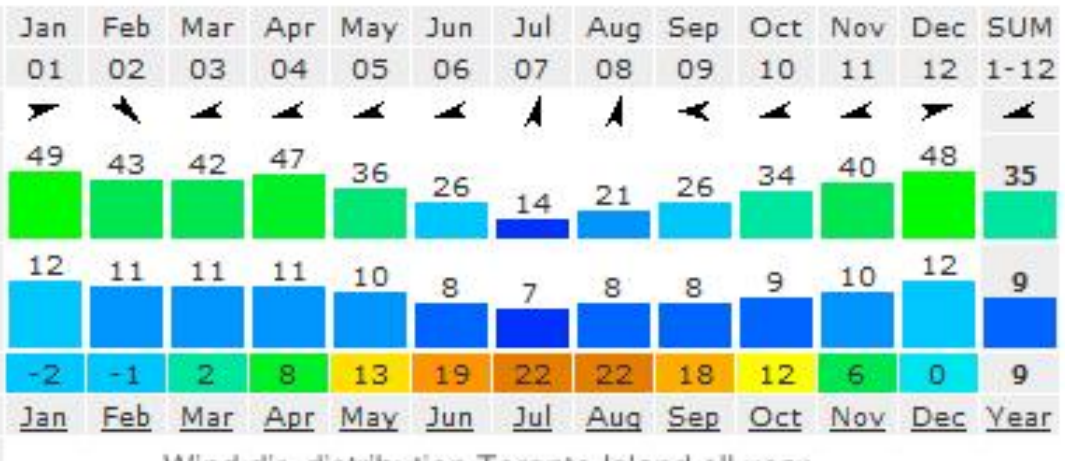

Wind dir. distribution Toronto Island all year

(1) windfinder.com

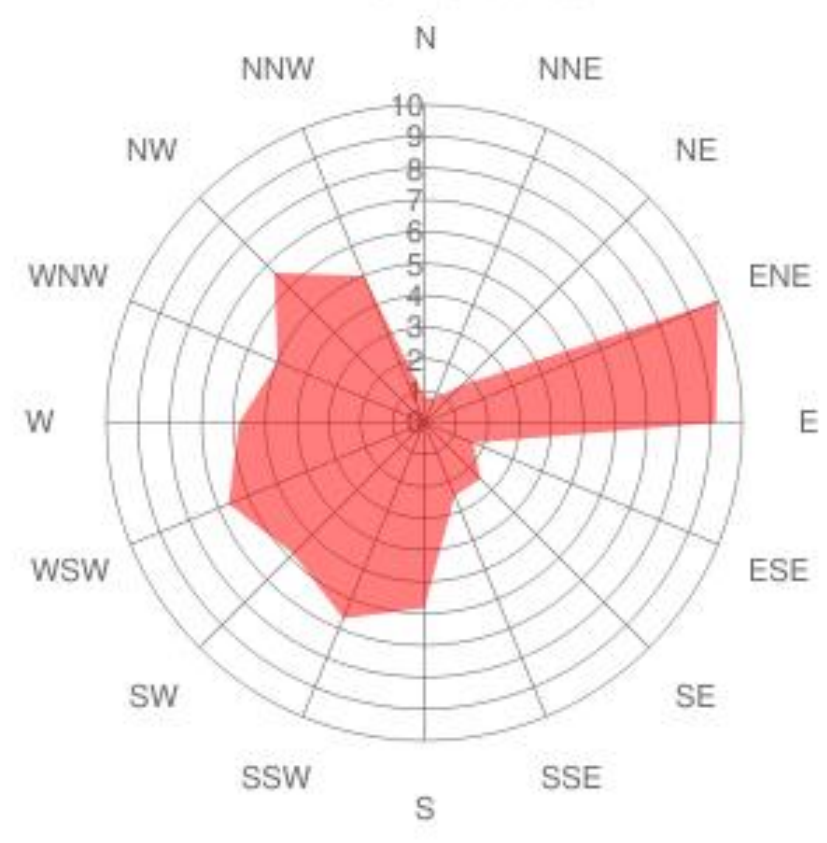

Figure 5.9: Toronto Island yearly average wind rose plot 4 


\section{Wind dir. distribution Toronto Island all year}

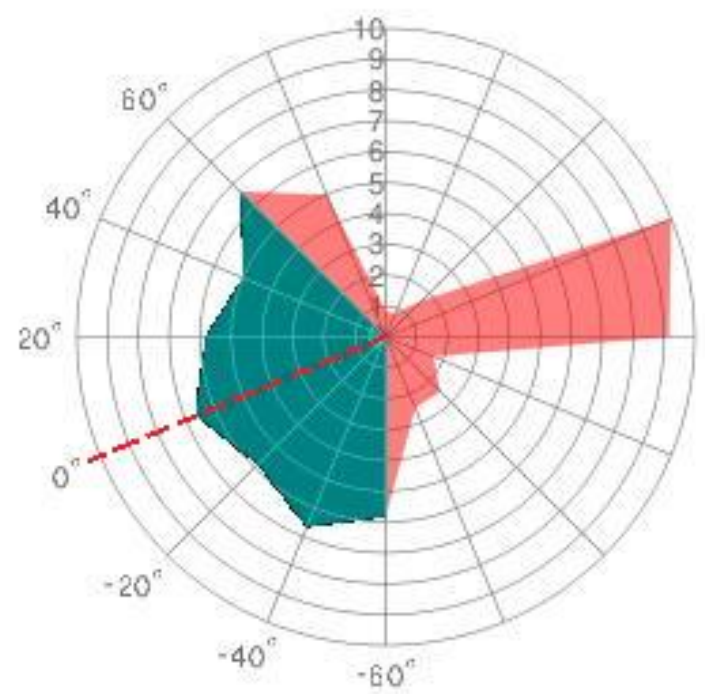

Figure 5.10: BAWT azimuth on wind rose plot [4]

0 , the power rating is highest for cases of 0 taper and produces a power rating of $35.6 \mathrm{~kW}$. The other ratings vary $13 \%$ and $34.8 \%$ for tapers of 0.33 and 0.66 respectively from the case of 0 taper.

The cases of tapered 0.33 and 0.5 spread factor is rated at $45.2 \mathrm{~kW}$ and case of 0.66 tapper and spread factor of 0.5 is rated at $44.6 \mathrm{~kW}$ with a difference of $1.3 \%$. The greater power rating developed by wind augmentation is counter balanced by a greater capture area.

For the case of 0.33 taper and spread factor of 1 the power rating is $25.8 \%$ higher than case of 0.66 taper at spread factor of 0.5 with equivalent Turbine areas. The velocity augmentation becomes the defining factor for energy capture rating. Comparing the rating for the taperred cases at their maximum spread factor and maximum turbine capture area, there is little increase in power rating. There is a $9.6 \%$ difference in power rating between the two configurations suggesting no real gains occur in power rating. 


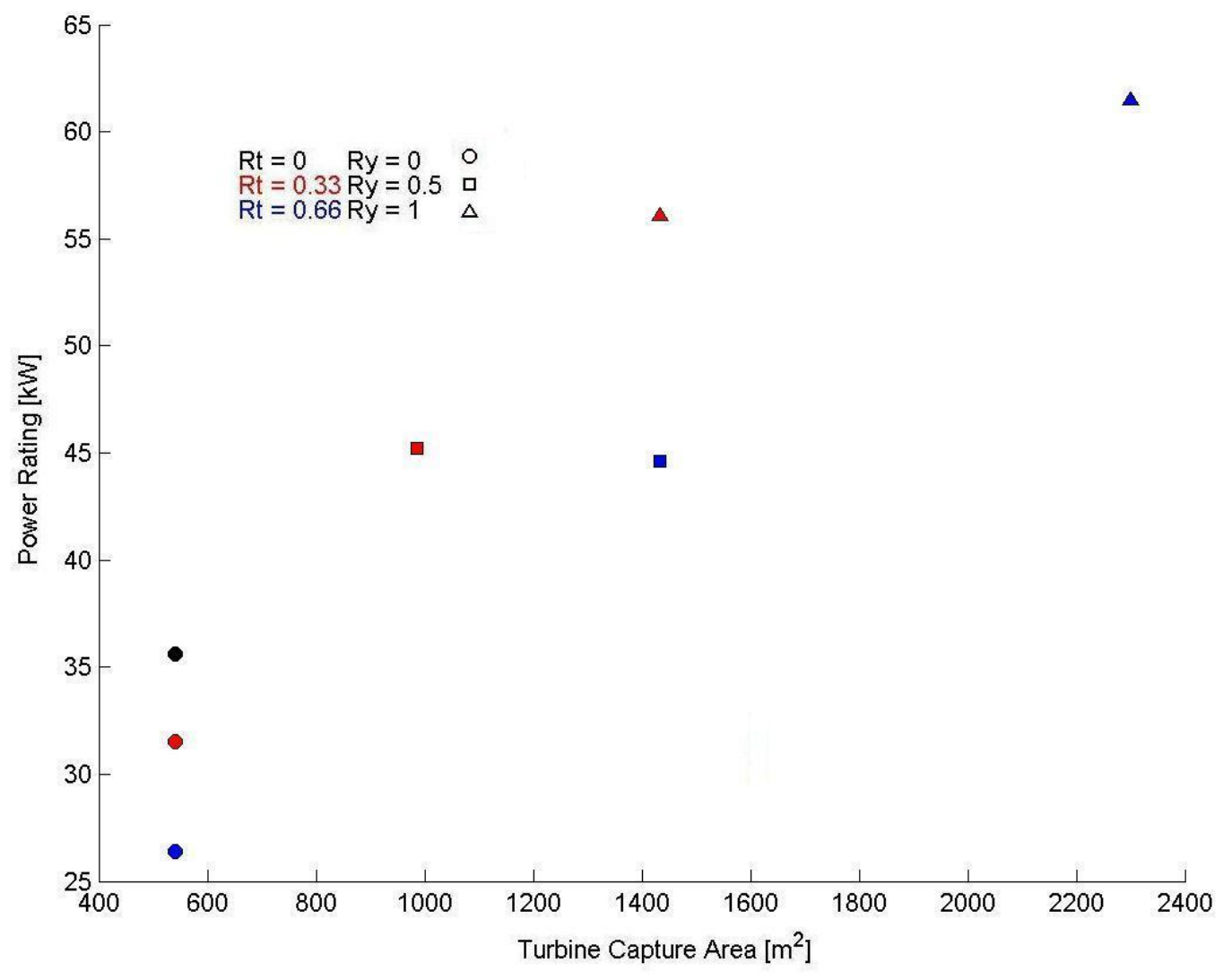

Figure 5.11: Power rating for Toronto Island case study

\begin{tabular}{c|c|c|c|c|c} 
& Turbine Area & \multicolumn{4}{|c}{ Wind Regime Power Rating $[\mathrm{kW}]$} \\
Taper & {$\left[\mathrm{m}^{2}\right]$} & High & Medium & Low & Toronto Island \\
\hline 0 & 540 & 84.4 & 95.1 & 103.1 & 35.6 \\
0.33 & 540 & 74.7 & 85.4 & 93.5 & 31.5 \\
0.33 & 986.7 & 105.9 & 130.3 & 145.7 & 45.2 \\
0.33 & 1433.5 & 132.3 & 166.5 & 190.2 & 56.1 \\
0.66 & 540 & 61.1 & 73.3 & 81.7 & 26.4 \\
0.66 & 1433.5 & 108.5 & 138.6 & 164.0 & 44.6 \\
0.66 & 2300.8 & 148.3 & 190.2 & 229.2 & 61.5
\end{tabular}

Table 5.3: Power rating for the wind regimes 


\section{Chapter 6}

\section{Conclusion}

It should be noted the simulated turbines was simplified using a porous jump boundary condition with a one-dimensional pressure drop approximation. Turbine turbulent factors such as rotational effects and tip losses were not taken into account within the simplified porous jump bounday.

The key aim of this study was to find a BAWT geometry which provides greatest velocity augmentation, total augmented power and overall power rating with respect to different wind regimes. This thesis utilized an optimized two dimensional BAWT footprint for low wind incidence angles derived from the work of Hans Hamm [14] where parabolic parameter definitions were imposed on the three dimensional extruding of the BAWT configuration.

Also important in this investigation was the modeling of ABL to obtain realistic flow characteristics. Proper modeling of the von Karman street developed the pressure cascade to influence the pressure upstream and ultimately the BAWT augmented power at the turbine.

In summary:

1. Augmentation factor correlates with continuity law and venturi effect

2. The BAWT configuration with no taper produced highest augmentation

3. The BAWT with greatest spread factor and taper produced lowest augmentation

4. Turbine capture area is the primary factor in generating best captured power

5. Velocity augmentation is a secondary factor in producing best capture power

6. Best configuration may lay between a taper ratio of 0.33 and 0.66 at max spread of 1

\subsection{Future Work}

Future analysis would be to model BAWT systems and include other building obstructions for the wind. Perhaps obstructions can act as a diverter or channel to provide consistent and high wind regime 
frequencies to BAWT. A realistic urban ABL maybe modeled for the study which may generate different BAWT profiles for greatest power augmentation.

Furthe study can also be made in varying the original gap size for greater range of turbine capture area. A trade of study of the gap area between the buildings and the turbine sweeping area and the number of turbines used can be done. Direct modelling of rotating turbines can generate the rotational effects and tip losses which occur in real flows across turbines.

The cross-section of the BAWT system can also be varied with height. The variation of the 2D parameters set by Hamm can be varied as a function of height and potentially a new parameter of twist may be incorporated. The design may also incorporate a bi-directional flow where the turbines can counter rotate and receive wind from the other end and generate power.

Lastly an optimization routine can be utilized in directing the researcher in the direction of the optimal BAWT configurations according to the imposed various constraints. 


\section{Appendix A}

\section{Parabolic BAWT Profile Formulation}

It was mentioned in the Methodology section the BAWT profile follows a parabolic profile for each of the three pamameters. This section derrives the the parabolic profile as a function of the buildings height. The base parabolic equation is defined as

$$
P(z)=a z^{2}+b z+c
$$

where $a, b$ and $c$ are constants to the parabolic equation. It was assumed there to be no change in the profile at the building roof 100m high, thus differentiating Equation A.1 and substituting the defined variables we get

$$
\begin{gathered}
\partial P(z)=2 a z+b \\
\partial P(z)=0 \quad @ \quad z=100 \\
\partial 0=2 a(100)+b \\
b=-200 a \\
P(z)=a z^{2}-200 b z+c
\end{gathered}
$$

Aslo, the parabolic profile is 0 at the ground when $\mathrm{z}=0 \mathrm{~m}$. Substituting the known variables into Equation A.2 


$$
\begin{array}{r}
P(z)=0 \quad @ \quad z=0 \\
c=0 \\
P(z)=a z^{2}-200 b z
\end{array}
$$

Also known is the roof top offset parameter $\chi \varnothing_{o}$ which from the referenced base diameter $\varnothing_{o}=20 \mathrm{~m}$ is substituted into Equation A.3

$$
\begin{aligned}
P(z)=20 \chi & @ z=100 \\
20 \chi & =a(100)^{2}-200(100) a \\
\chi & =-500 a \\
a & =-\frac{\chi}{500}
\end{aligned}
$$

Substituting $a$ back into Equation A.3

$$
\begin{gathered}
P(z)=-\left(\frac{\chi}{500}\right) z^{2}+200\left(\frac{\chi}{500}\right) z \\
=\frac{z(200-z)}{500} \chi \\
P(z)=\frac{z(200-z)}{500} \chi
\end{gathered}
$$

Equation A.4 is the derived parabolic profile defining the BAWT taper and forward lean parameters. Spread factor has an added constant at the end of A.4 which defines the initial gap of $3 \mathrm{~m}$ set by the refferenced model of Hans Hamm. Thus the parabolic function for spread factor is given as

$$
P(z)=\frac{z(200-z)}{500} \chi+3
$$




\section{Appendix B}

\section{Bodies of Influence}

Bodies of influence are used to control element sizing at specified locations within the control volume without modeling an internal body. Two bodies of influence were used to define the vortex street with varying element sizes of influence. The sizes were varied from finest located up the center of the of BAWT system with a gradual coarsening in the bodies of influent as they move further downstream in the domain.

A body of influence of $120 \mathrm{~m}$ diameter encapsulated the BAWT system and was extended downstream to the outlet area to capture the von Karman street. Figure B.1 shows the bodies of influence encapsulating the BAWT system as domed structures which are stretched downstream in the von Karman street.

The length of the medium body of influence was defined by the assumption vortex would dissipate enough kinetic energy and extend turbulent length scale to be captured in the coarser body of influence after three shedding periods. The frequency of shedding was determined from the Strouhal numbers defined by Zdrakovich for flows over cylinders at flow Reynolds nuber $1 \cdot 10^{6}$ defined in Table B.1.

The distance a vortex propagates from a body after three periods is found by multiplying the inverse of frequency and the potential wind speed define in Equation B.1.

$$
l_{v p}=f_{r}^{-1} u_{p}
$$

Table B.1 shows the resultant vortex propagation distances from a cylinder using flow Strouhal num-

\begin{tabular}{c|c|c|c}
$\begin{array}{c}\text { Strouhal } \\
\text { Numbers }\end{array}$ & $\begin{array}{c}\text { Frequency } \\
{[\mathrm{Hz}]}\end{array}$ & $\begin{array}{c}\text { Periods } \\
{[\mathrm{s}]}\end{array}$ & $\begin{array}{c}\text { Propagation } \\
\text { Distance }[\mathrm{m}]\end{array}$ \\
\hline 0.18 & 0.0374 & 26.7 & 334 \\
0.50 & 0.1040 & 9.6 & 120
\end{tabular}

Table B.1: Vortex propagation distances after three periods with respect to Strouhal numbers 


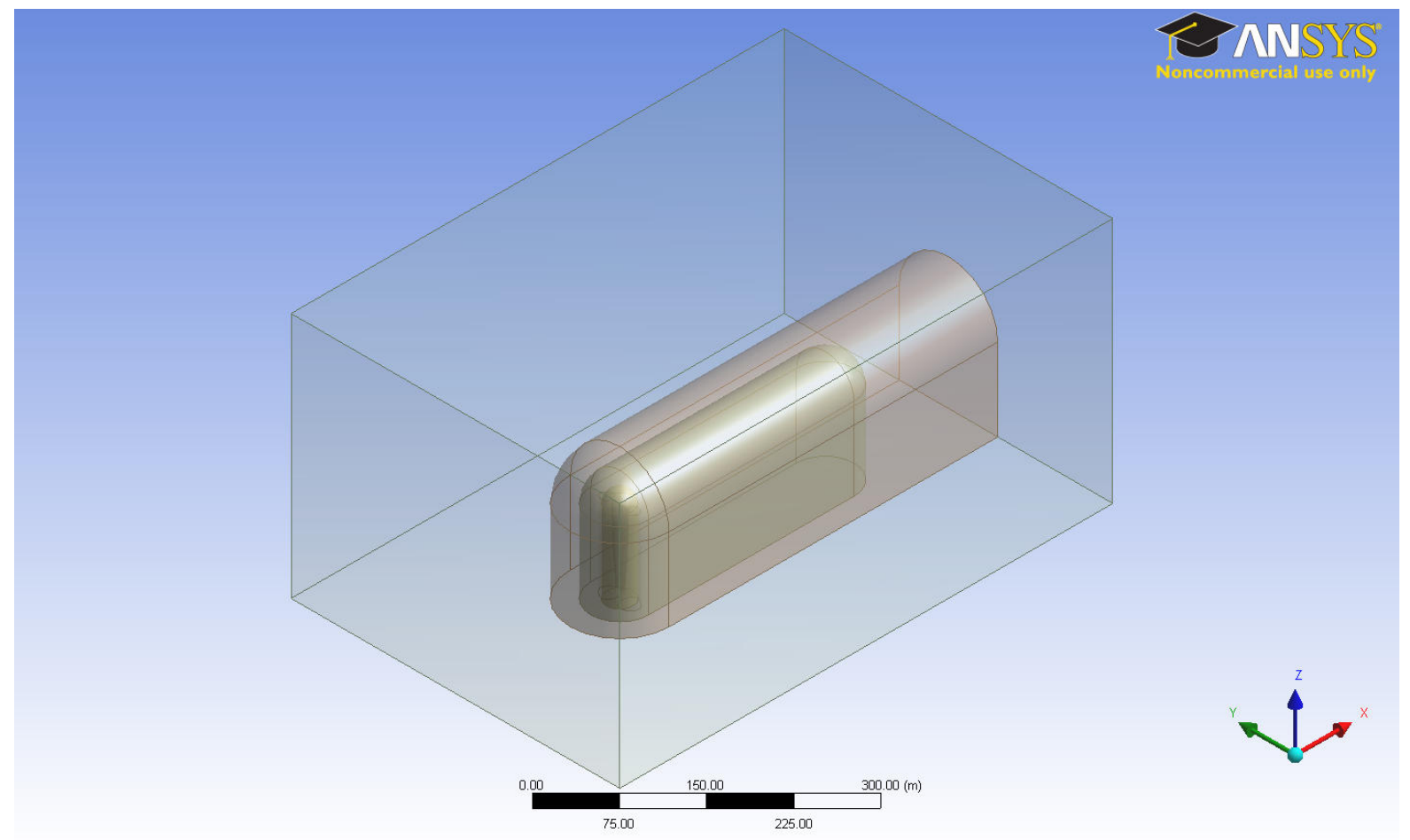

Figure B.1: Body of influence 
bers. Zdravkovich defines Strouhal numbers to fall within the range from $0.18-0.50$ which is dependent on the turbulence model chosen for analysis.

Since there is a range of Strouhal numbers it was assumed any number may appear within this study. Therefore the largest propagation distance was used which corresponds to the lowest shedding frequency from a Strouhal number of 0.18 .

Figures B.2 and B.3 show the turbulent von Karman street formed behind the BAWT systems. They depict vortex bubbles to remain within the defined bodies of influence for the modelling of the upstream pressure cascade.

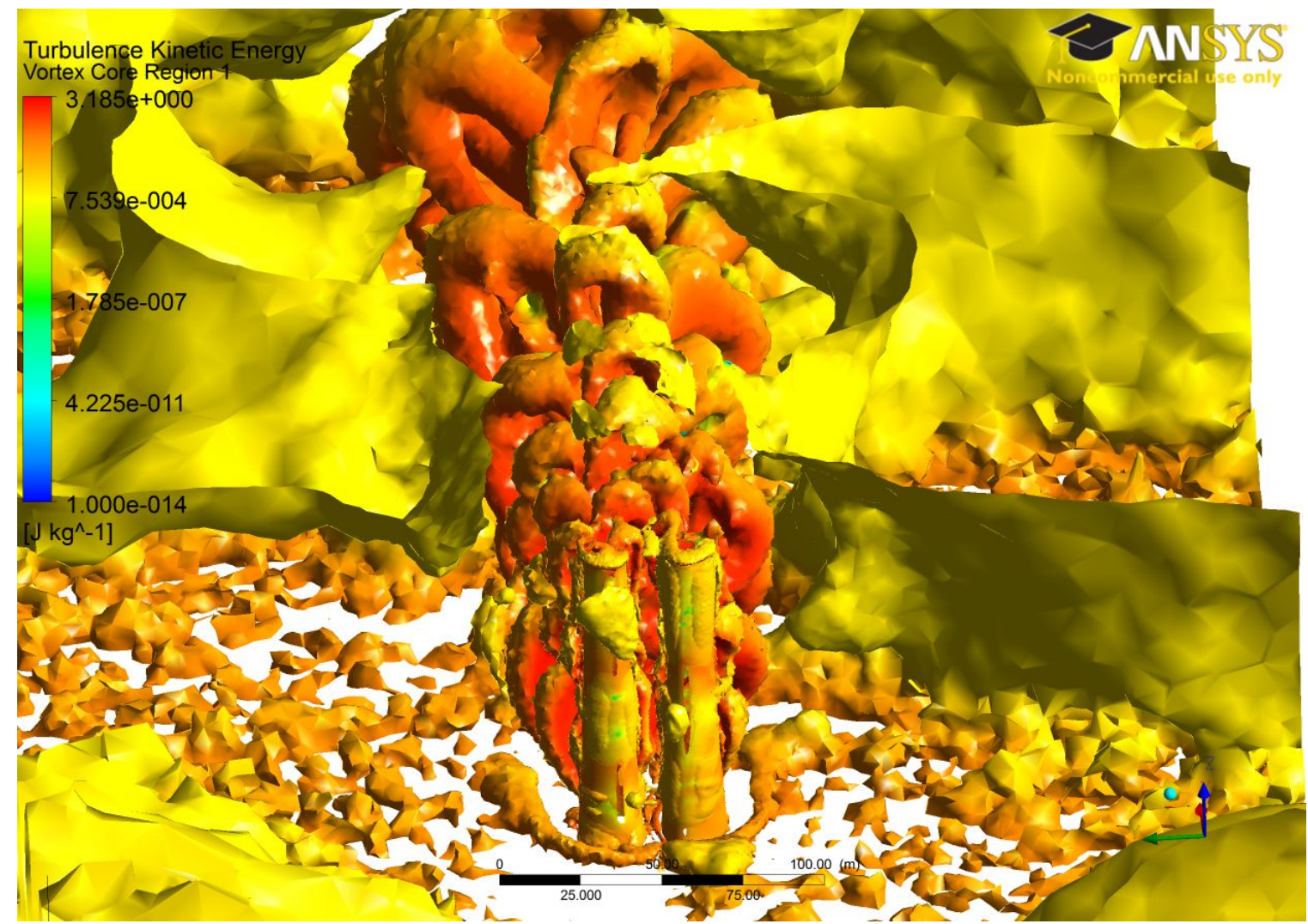

Figure B.2: Q-Criterion Iso Surface with Turbulent kinetic energy variations $\left(R_{t}=0.33, R_{x}=0.5\right.$, $R_{y}=0.5$ and $\alpha=0^{\circ}$

Also in the two Figures B.2 and B.3. three separation bubbles are visibily defined within the $330 \mathrm{~m}$ length of the Fine Body of Influence. Thus it can be said the $k-\omega$ (SST) model, near-wall modeling and vortex shedding produces a pressure cascade well enough for the purpose of this study. 


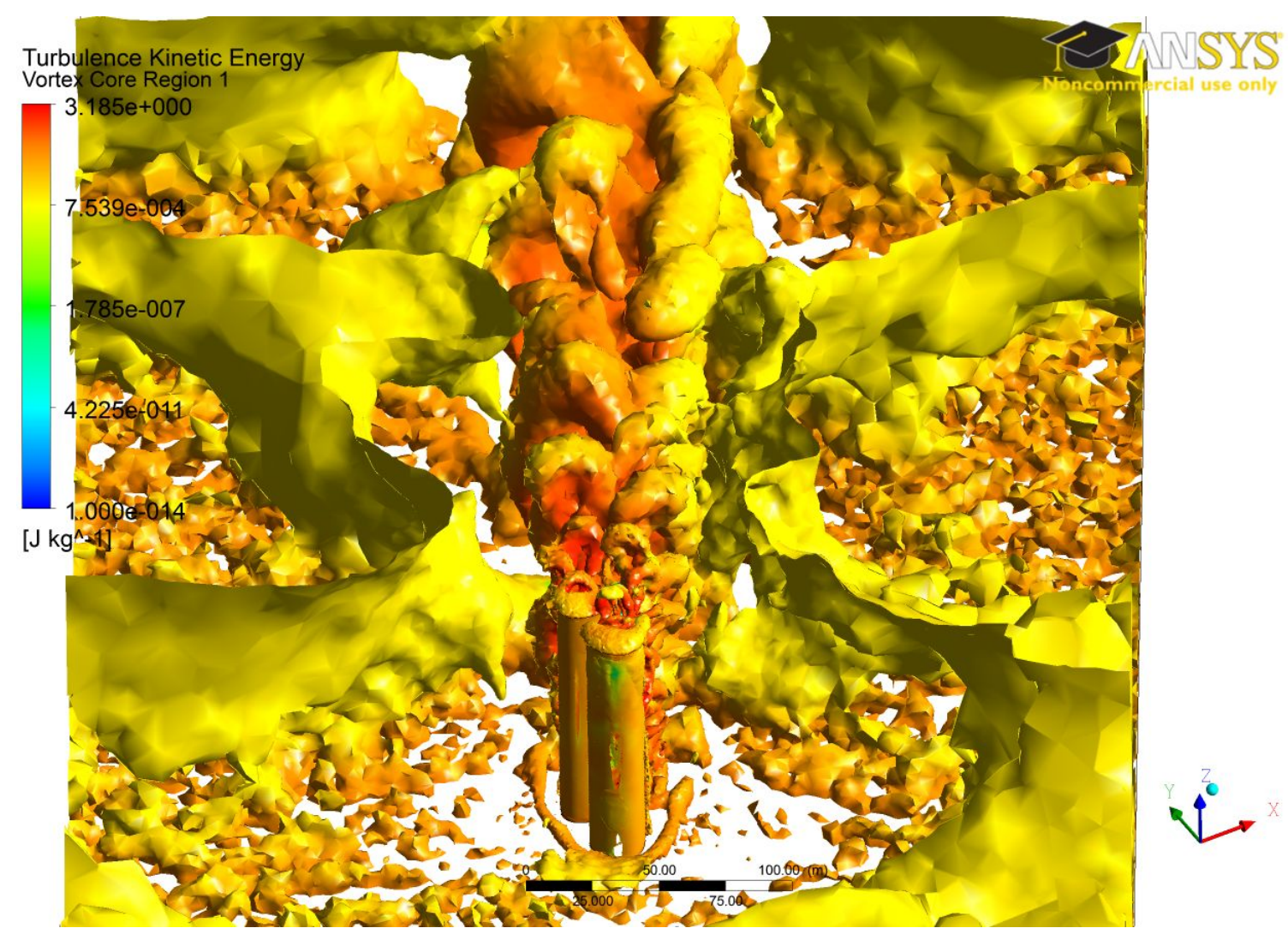

Figure B.3: Q-Criterion Iso Surface with Turbulent kinetic energy variations $\left(R_{t}=0\right.$ and $\alpha=60^{\circ}$ 


\section{Appendix $\mathrm{C}$}

\section{Porous Jump}

Turbines were modeled using the porous jump boundary condition at the Turbine boundary. The NavierStokes equations with the addition of momentum source terms where used to model the porous media. The Navier-Stokes equations with momentum sources terms are given below in Cartesian coordinates in Equation C.1

$$
\Delta P=-\left(\frac{\mu}{\alpha} \nu+C_{2} \frac{1}{2} \rho u^{2}\right) \Delta m
$$

$\mu$ is the fluid viscosity, $\nu$ is the dynamic viscosity, $\alpha$ is the permeability of the porous media. Two source terms account for the viscous losses and inertial loss when the fluid passes through the porous jump Turbine boundary condition. The first term is called the Darcy term which is the instantaneous discharge rate of a fluid through a porous medium.

The simplification does not incorporate the effects and flow characteristics of the vortex shedding created by the rotating blades, thus omitting three dimensional wake and tip effects. The simplified modeled turbine consists of no porous media therefore no viscous losses incur thus the Darcy term is dropped and porous jump is defined by Equation C.2.

$$
\Delta P=-C_{2} \frac{1}{2} \rho u^{2} \Delta m
$$

To maintain consistency with the work from Hans Hamm [14] his arbitrary pressure coefficient $C_{2}=$ 0.4 was used for the pressure loss coefficient of the porous jump with a unit length of $\Delta m=1$.

Figure C.1 illustrates the pressure drop though the mid section of the BAWT along the $x$-axis at a height of $60 \mathrm{~m}$. The pressure drop accros the turbine was measured from the difference of the measured pressures at the Tubine capture area and $1 \mathrm{~m}$ downstream. The pressure difference of the cell values are calculated to be $\Delta P=-11.49 \mathrm{~Pa}$.

Verification of the pressure drop found at the porous section is based on the fluid velocity entering the porous jump boundary and was measured to be $6.71 \mathrm{~m} / \mathrm{s}$. Using equation C.2 the pressure drop 


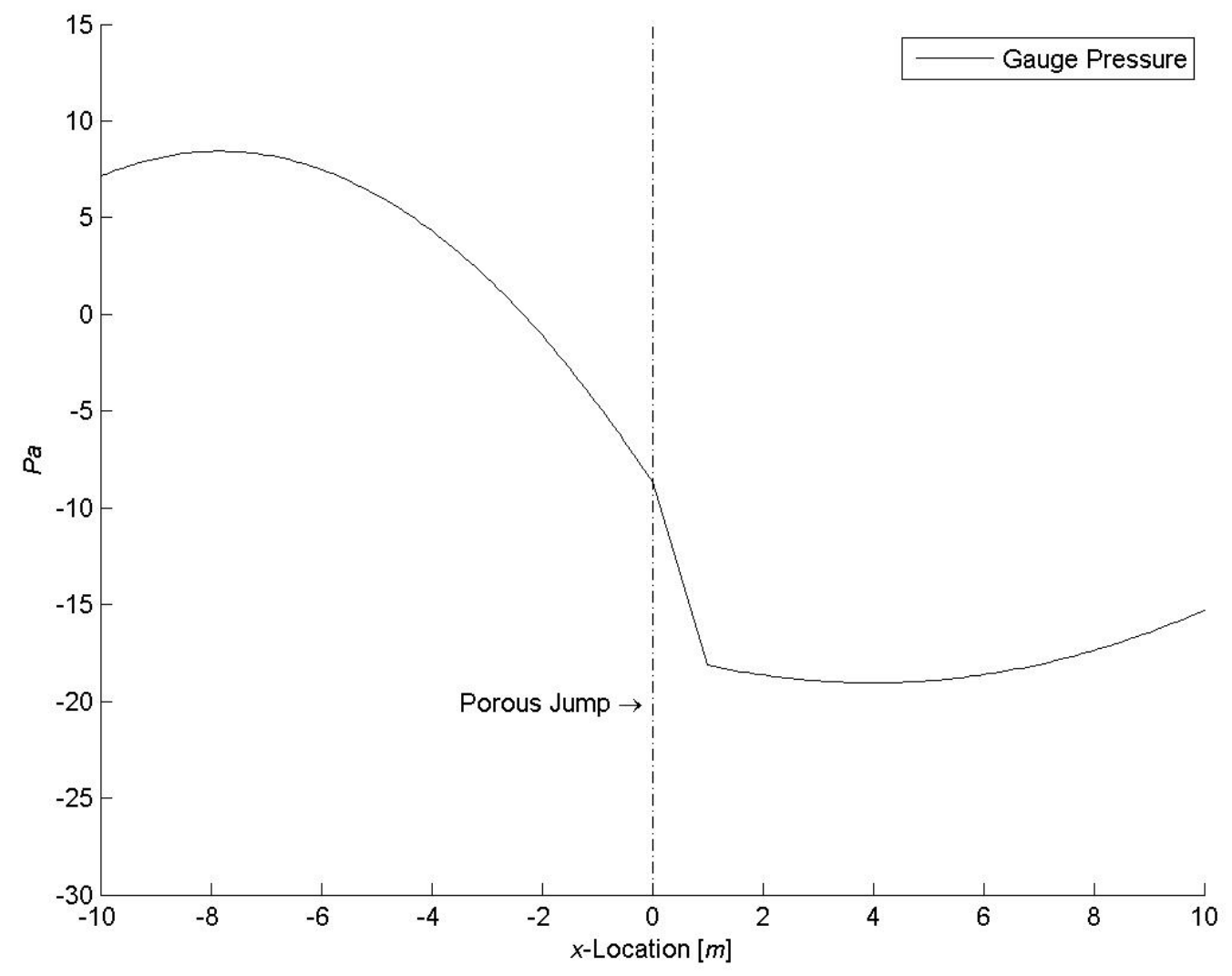

Figure C.1: Pressure reading through BAWT a model 
across the porous media was found to be $-11.04 \mathrm{~Pa}$ with a difference of $4.1 \%$ from the measured value.

$$
\begin{aligned}
\Delta P & =-\left(0.4 \frac{1}{2}(1.225)(6.713)^{2}\right)(1) \\
& =-11.04 P a
\end{aligned}
$$





\section{Appendix D}

\section{Figures}

This section shows the pressure contours on the BAWT Walls, Ground and a pressure contour of the center plane through the BAWT projected on the fall boundary Side boundary. Also stream lines depict the turbulence aft of the BAWT.

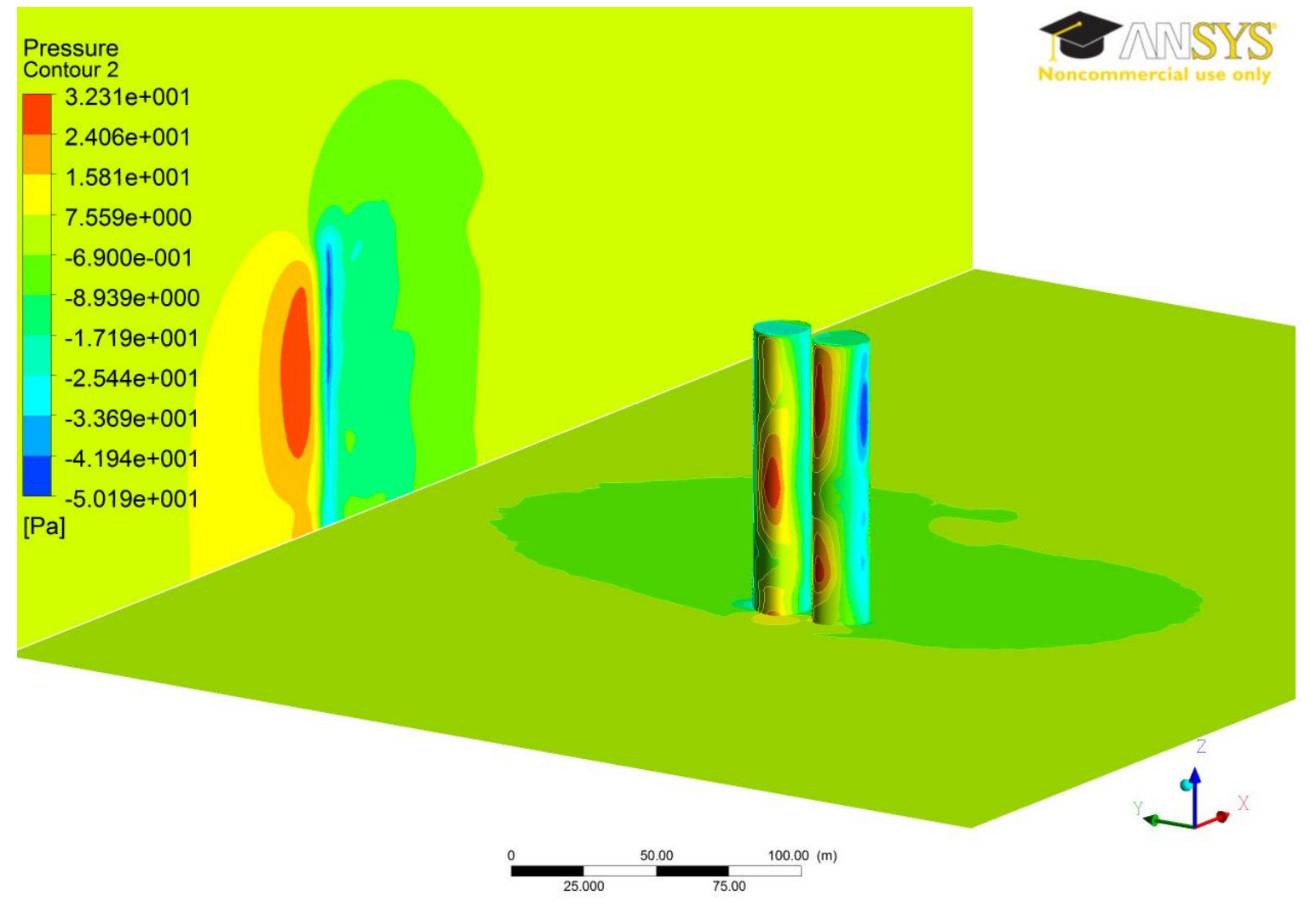

Figure D.1: Contour of pressure on BAWT walls the pressure contour through the mid-plane projected on the domain side wall 


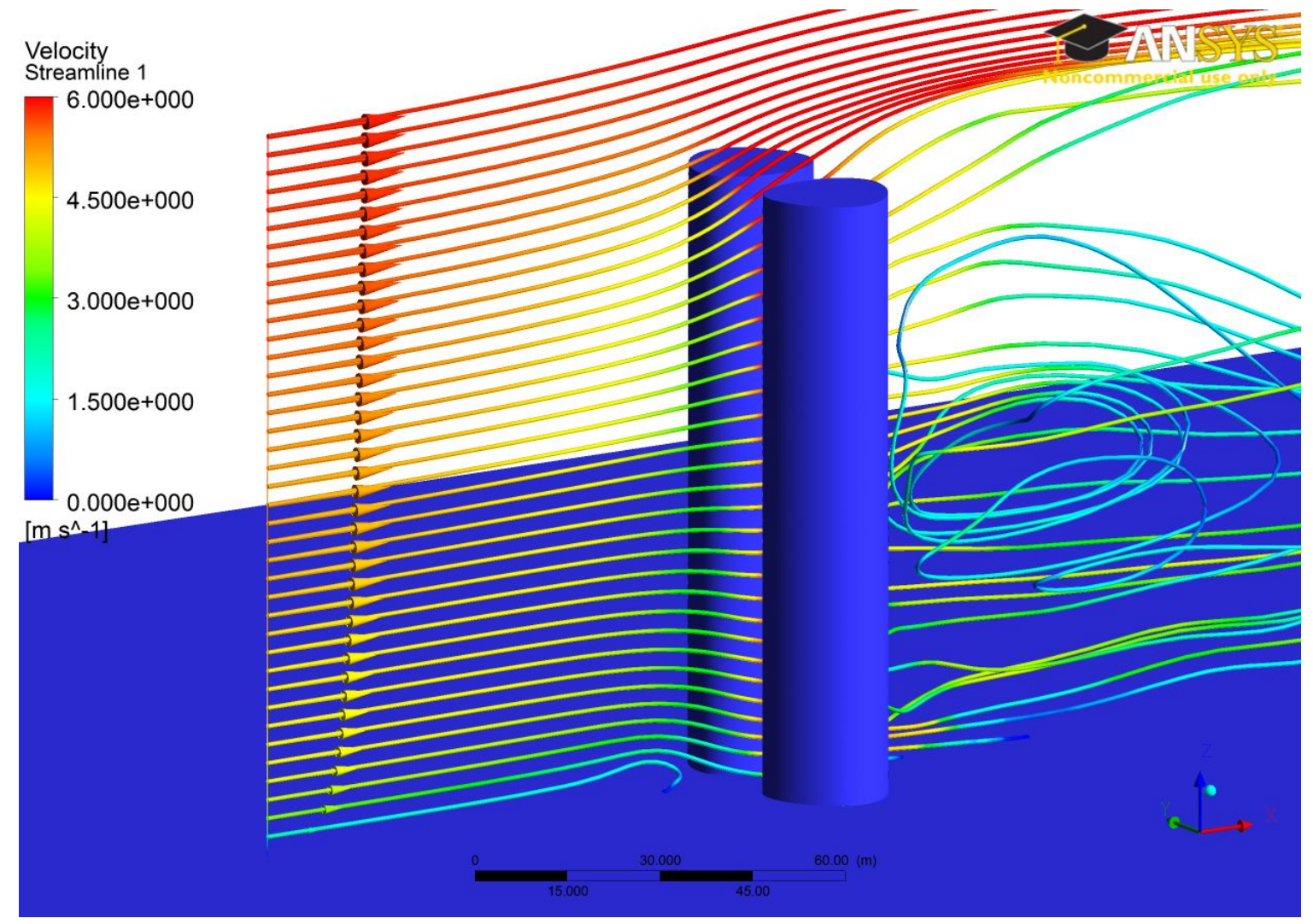

Figure D.2: Flow stream lines $\left(R_{t}=0, R_{x}=0, R_{y}=0\right.$ and $\left.\alpha=0\right)$ 


\section{Appendix E}

\section{Velocity Inlet ABL UDF}

This section shows the user defined function (UDF) for FLUENT created to define the ABL for the velocity inlet condition at the Inlet boundary.

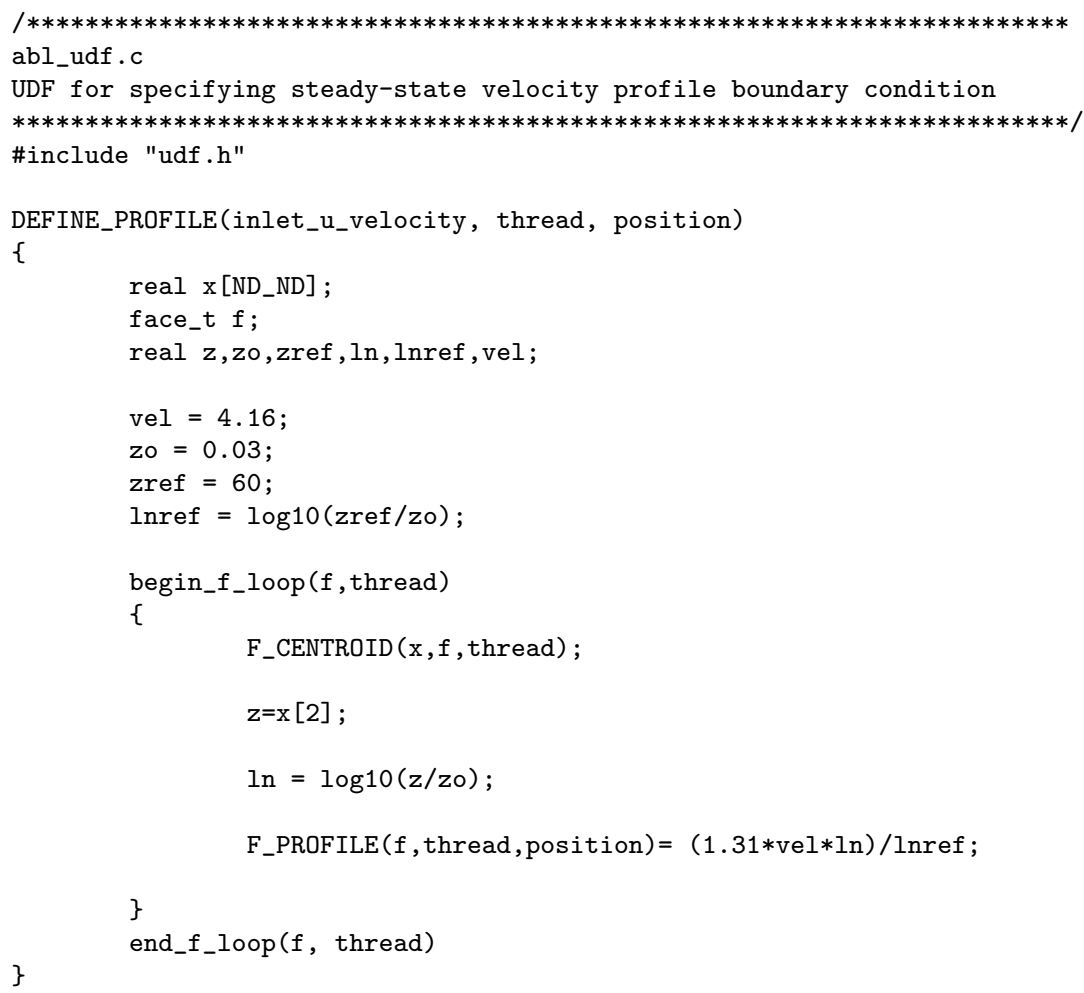





\section{Appendix F}

\section{FLUENT Journal Scripting}

The journal scrip was create to submit bash jobs to FLUENT for processing. The journal script defined the BAWT configurations used and the model set up and solution configurations.

/file/set-batch-options no yes no /file/read-case SGE_O_WORKDIR/MeshFiles/Rt_RT/Rx_RX_Ry_RY/FFF.Rt_RT_Rx_RX_Ry_RY_AOA_ANGLE.msh

/define/user-defined/compiled-functions compile SGE_O_WORKDIR/SUBDIR/Iibudf yes SGE_O_WORKDIR/SUBDIR/ libudf/abl_udf.c "" SGE_0_WORKDIR/SUBDIR/libudf/abl_udf.c ""

/define/user-defined/compiled-functions load SGE_0_WORKDIR/SUBDIR/libudf

/define/custom-field-functions/define "abl" VIN*1.31*log10((z_coordinate)/0.03)/log10(60/0.03)

/define/custom-field-functions/define "power_aug" $1 / 2 *$ density*(x_velocity) - 3

/define/custom-field-functions/define "power_abl" 1/2*density*(abl)^3

/define/custom-field-functions/define "initial_u_velocity" abl*cos(ANGLE*PI/180)

/define/custom-field-functions/define "initial_v_velocity" abl*sin(ANGLE*PI/180)

/define/models/solver/density-based-implicit yes

/define/models/energy yes

/define/models/viscous kw-sst yes

/define/boundary-conditions/velocity-inlet inlet no no yes yes yes yes "udf" "inlet_u_velocity::libudf" no 300 no yes 101 no 0

/define/boundary-conditions/pressure-outlet outlet no 0 no 300 no no yes yes no 0.7 no 1 no no

/define/boundary-conditions/wall ground 0 no 0 no no no 0 no no no 0.03 no 0.5

/define/boundary-conditions/wall leftbuilding 0 no 0 no no no 0 no no no 0 no 0.5

/define/boundary-conditions/copy-bc leftbuilding rightbuilding leftbuildingtop rightbuildingtop ()

/solve/set/discretization-scheme/amg-c 1

/solve/set/discretization-scheme/k 1

/solve/set/discretization-scheme/omega 1

/solve/set/under-relaxation/k 0.8

/solve/set/under-relaxation/omega 0.8

/solve/set/under-relaxation/turb-viscosity 1

/solve/monitors/residual/criterion-type 1

/solve/monitors/residual monitor yes yes yes yes yes yes yes

/solve/set/flow-warnings no

/solve/initialize/set-defaults k 0.7

/solve/initialize/set-defaults omega 1

/solve/initialize/set-defaults temperature 300

/solve/initialize/initialize-flow

/solve/patch Model-fluid () x-velocity no yes initial_u_velocity

/solve/patch Model-fluid () y-velocity no yes initial_v_velocity

/solve/initialize/set-fmg-initialization 50.001100 .001500 .0015000 .0015000 .0011000 .75 yes

/solve/initialize/fmg-initialization yes

/solve/iterate 50

/define/boundary-conditions/modify-zones zone-type turbine porous-jump 
/define/boundary-conditions/porous-jump turbine $1 \mathrm{e}+1010.4$

/solve/iterate 100

/define/models/unsteady-2nd-order yes

/solve/monitors/surface/set-monitor $\mathrm{x}$-velocity-turbineinlet "Area-Weighted Average" $\mathrm{x}$-velocity turbine () no no yes "ave_x_velocity_-turbineinlet_area_weighted" 1 yes flow

/solve/monitors/surface/set-monitor abl-velocity-turbineinlet "Area-Weighted Average" abl turbine () no no yes "ave_abl_velocity_turbineinlet_area_weighted" 1 yes flow

/solve/monitors/surface/set-monitor mass-flow-rate-turbineinlet "Mass Flow Rate" turbine () no no yes "mass_flow_rate_turbineinlet" 1 yes flow

/solve/monitors/surface/set-monitor augmented-power "Integral" power_aug turbine () no no yes "power_aug" 1 yes flow

/solve/monitors/surface/set-monitor non-augmented-power "Integral" power_abl turbine () no no yes "power_abl" 1 yes flow

/file/auto-save data-frequency 200

/solve/set time-step TIME_STEP

/solve/dual-time-iterate N_TIME_STEPS N_ITERATIONS

/file/write-case Rt_RT_Rx_RX_Ry_RY_AOA_ANGLE.cas.gz

/file/write-data Rt_RT_Rx_RX_Ry_RY_AOA_ANGLE.dat.gz

/report/summary yes "report.sum"

/exit 


\section{Appendix G}

\section{Shell Bash Script}

Shell scripts were created to automate the journal scritp variations defining the solution criteria for FLUENT and aswell the submition of bash cases.

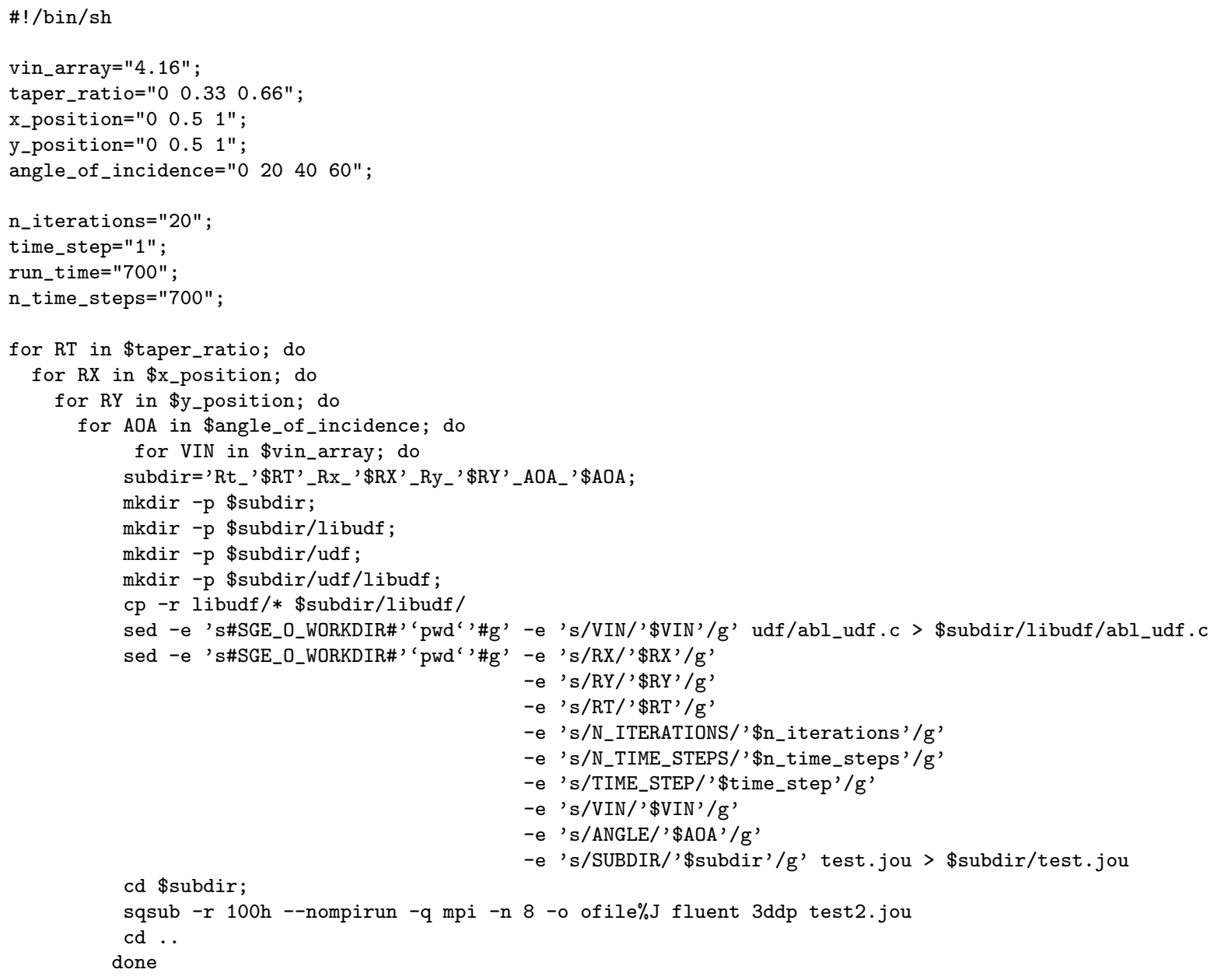




done
done
done
done




\section{Appendix $\mathbf{H}$}

\section{FLUENT CFD Code Settings}

A sample results file produced by FLUENT describing the CFD code settings.

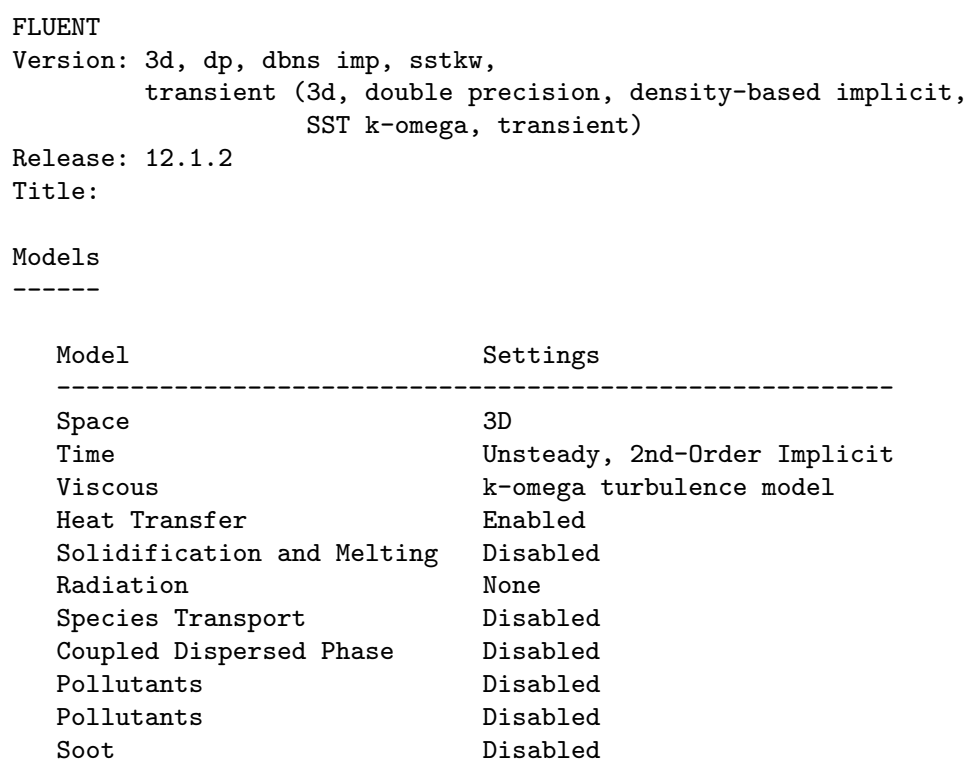

Space 3 3D

Material Properties

\begin{tabular}{|c|c|c|c|}
\hline Material: air (fluid) & & & \\
\hline Property & Units & Method & Value(s) \\
\hline Density & $\mathrm{kg} / \mathrm{m} 3$ & constant & 1.225 \\
\hline Cp (Specific Heat) & $\mathrm{j} / \mathrm{kg}-\mathrm{k}$ & constant & 1006.43 \\
\hline Thermal Conductivity & $\mathrm{w} / \mathrm{m}-\mathrm{k}$ & constant & 0.0242 \\
\hline Viscosity & $\mathrm{kg} / \mathrm{m}-\mathrm{s}$ & constant & $1.7894 \mathrm{e}-05$ \\
\hline Molecular Weight & $\mathrm{kg} / \mathrm{kgmol}$ & constant & 28.966 \\
\hline Thermal Expansion Coefficient & $1 / \mathrm{k}$ & constant & 0 \\
\hline Speed of Sound & $\mathrm{m} / \mathrm{s}$ & none & $\# f$ \\
\hline
\end{tabular}


Material: aluminum (solid)

$\begin{array}{llll}\text { Property } & \text { Units } & \text { Method } & \text { Value(s) } \\ & \mathrm{kg} / \mathrm{m} 3 & \text { constant } & 2719 \\ \text { Density } & \mathrm{j} / \mathrm{kg}-\mathrm{k} & \text { constant } & 871 \\ \text { Cp (Specific Heat) } & \mathrm{w} / \mathrm{m}-\mathrm{k} & \text { constant } & 202.4 \\ \text { Thermal Conductivity } & & \end{array}$

Cell Zone Conditions

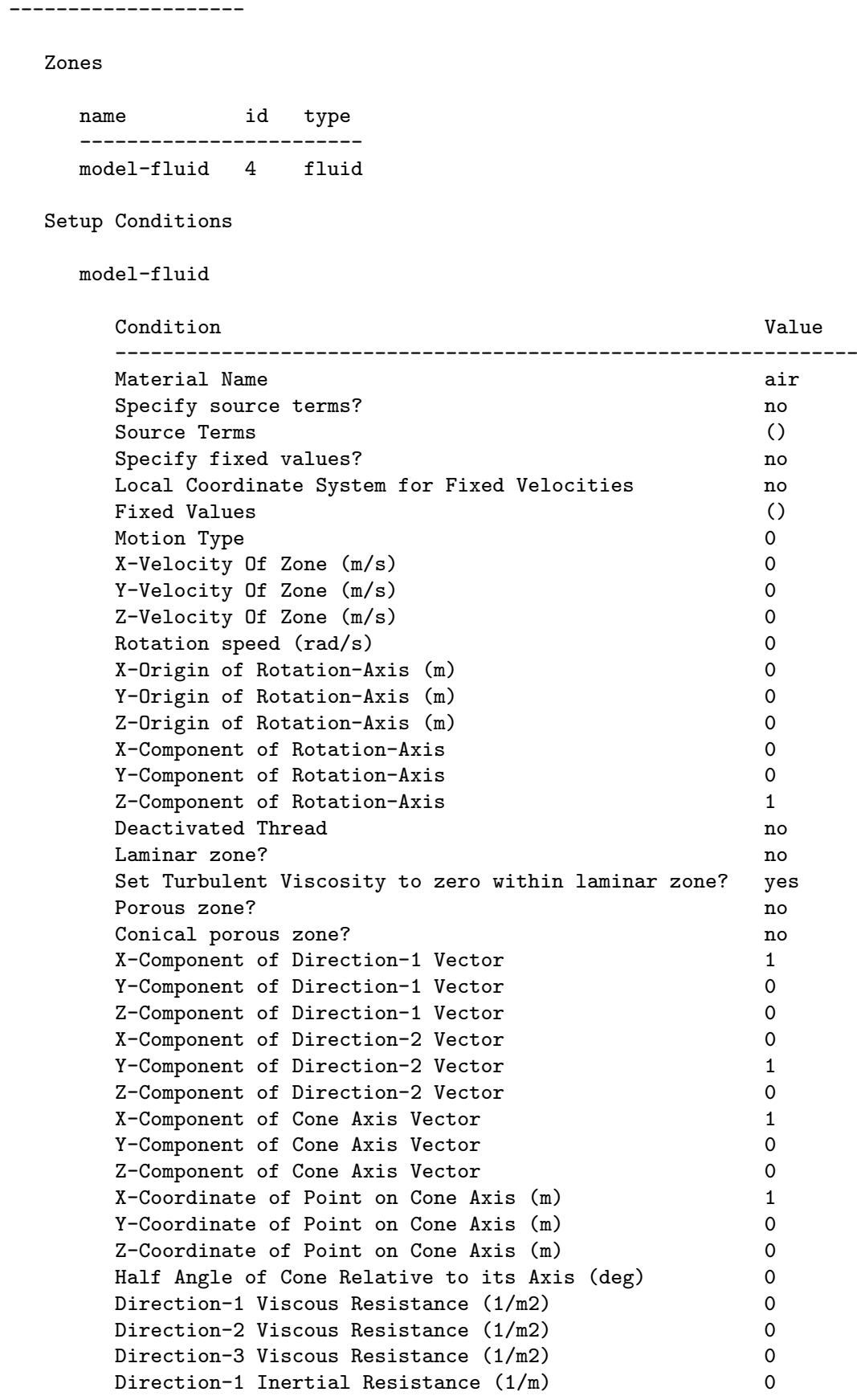


Direction-2 Inertial Resistance (1/m)

Direction-3 Inertial Resistance $(1 / \mathrm{m})$

Co Coefficient for Power-Law

C1 Coefficient for Power-Law

Porosity

Solid Material Name

0
0
0
0
1
aluminum

Boundary Conditions

$\begin{array}{lll}\text { Zones } & & \\ & & \\ \text { name } & \text { id } & \text { type } \\ \text {-------------------- } \\ \text { turbine } & 3 & \text { porous-jump } \\ \text { ground } & 7 & \text { wall } \\ \text { inlet } & 8 & \text { velocity-inlet } \\ \text { leftbuilding } & 9 & \text { wall } \\ \text { leftbuildingtop } & 10 & \text { wall } \\ \text { outlet } & 11 & \text { pressure-outlet } \\ \text { rightbuilding } & 12 & \text { wall } \\ \text { rightbuildingtop } & 13 & \text { wall } \\ \text { symmetry } & 14 & \text { symmetry }\end{array}$

Setup Conditions

turbine

\begin{tabular}{ll} 
Condition & Value \\
\hline$-y c e$ Permeability (m2) & $1 \mathrm{e}+10$ \\
Porous Medium Thickness (m) & 1 \\
Pressure-Jump Coefficient (C2) (1/m) & 0.4
\end{tabular}

ground

$\begin{array}{ll}\text { Condition } & \text { Value } \\ \text { Wall Thickness (m) } & 0 \\ \text { Heat Generation Rate (w/m3) } & 0 \\ \text { Material Name } & \text { aluminum } \\ \text { Thermal BC Type } & 1 \\ \text { Temperature (k) } & 300 \\ \text { Heat Flux (w/m2) } & 0 \\ \text { Convective Heat Transfer Coefficient (w/m2-k) } & 0 \\ \text { Free Stream Temperature (k) } & 300 \\ \text { Wall Motion } & 0 \\ \text { Shear Boundary Condition } & 0 \\ \text { Define wall motion relative to adjacent cell zone? } & \text { yes } \\ \text { Apply a rotational velocity to this wall? } & \text { no } \\ \text { Velocity Magnitude (m/s) } & 0 \\ \text { X-Component of Wall Translation } & 1 \\ \text { Y-Component of Wall Translation } & 0 \\ \text { Z-Component of Wall Translation } & 0 \\ \text { Define wall velocity components? } & \text { no } \\ \text { X-Component of Wall Translation (m/s) } & 0 \\ \text { Y-Component of Wall Translation (m/s) } & 0 \\ \text { Z-Component of Wall Translation (m/s) } & 0 \\ \text { External Emissivity } & 1 \\ \text { External Radiation Temperature (k) } & 300 \\ \text { Wall Roughness Height (m) } & 0.03 \\ \text { Wall Roughness Constant } & 0.5 \\ & \end{array}$


Rotation Speed ( $\mathrm{rad} / \mathrm{s}$ )

X-Position of Rotation-Axis Origin (m) Y-Position of Rotation-Axis Origin (m)

Z-Position of Rotation-Axis Origin (m)

$\mathrm{X}$-Component of Rotation-Axis Direction

Y-Component of Rotation-Axis Direction

Z-Component of Rotation-Axis Direction

$\mathrm{X}$-component of shear stress (pascal)

Y-component of shear stress (pascal)

Z-component of shear stress (pascal)

Surface tension gradient $(\mathrm{n} / \mathrm{m}-\mathrm{k})$

Specularity Coefficient

0
0
0
0
0
0
1
0
0
0
0
0

inlet

Condition Value

Velocity Specification Method 2

Reference Frame 0

Velocity Magnitude (profile udf inlet_u_velocity::libudf)

Coordinate System 0

$\mathrm{X}$-Velocity $(\mathrm{m} / \mathrm{s})$

Y-Velocity $(\mathrm{m} / \mathrm{s})$

Z-Velocity $(\mathrm{m} / \mathrm{s})$

Y-Component of Flow Direction

Z-Component of Flow Direction 0

$\mathrm{X}$-Component of Axis Direction 1

Y-Component of Axis Direction 0

Z-Component of Axis Direction 0

$\mathrm{X}$-Coordinate of Axis Origin (m) 0

Y-Coordinate of Axis Origin (m) 0

Z-Coordinate of Axis Origin (m) 0

Angular velocity ( $\mathrm{rad} / \mathrm{s}) \quad 0$

Temperature (k) 300

Turbulent Specification Method 1

Turbulent Kinetic Energy (m2/s2) 1

Specific Dissipation Rate (1/s) 1

Turbulent Intensity (\%) 10

Turbulent Length Scale (m) 1

Hydraulic Diameter (m) 1

Turbulent Viscosity Ratio 1

Outflow Gauge Pressure (pascal) 0

is zone used in mixing-plane model? no

leftbuilding

Condition Value

Wall Thickness (m) 0

Heat Generation Rate (w/m3) 0

Material Name aluminum

Thermal BC Type 1

Temperature (k) 300

Heat Flux (w/m2) 0

Convective Heat Transfer Coefficient (w/m2-k) 0

Free Stream Temperature (k) 300

Wall Motion 0

Shear Boundary Condition 0

Define wall motion relative to adjacent cell zone? yes

Apply a rotational velocity to this wall? no

Velocity Magnitude (m/s) 0 
X-Component of Wall Translation 1 Y-Component of Wall Translation 0

Z-Component of Wall Translation 0

Define wall velocity components? no

$\mathrm{X}$-Component of Wall Translation (m/s) 0

Y-Component of Wall Translation $(\mathrm{m} / \mathrm{s}) \quad 0$

Z-Component of Wall Translation (m/s) 0

External Emissivity 1

External Radiation Temperature (k) 300

Wall Roughness Height (m) 0

Wall Roughness Constant 0.5

Rotation Speed ( $\mathrm{rad} / \mathrm{s})$

$\mathrm{X}$-Position of Rotation-Axis Origin (m) 0

Y-Position of Rotation-Axis Origin (m) 0

Z-Position of Rotation-Axis Origin (m) 0

$\mathrm{X}$-Component of Rotation-Axis Direction 0

Y-Component of Rotation-Axis Direction 0

Z-Component of Rotation-Axis Direction 1

$\mathrm{X}$-component of shear stress (pascal) 0

Y-component of shear stress (pascal) 0

Z-component of shear stress (pascal) 0

Surface tension gradient $(\mathrm{n} / \mathrm{m}-\mathrm{k})$

Specularity Coefficient 0

leftbuildingtop

Condition Value

Wall Thickness (m) 0

Heat Generation Rate (w/m3) 0

Material Name aluminum

Thermal BC Type 1

Temperature (k) 300

Heat Flux (w/m2)

Convective Heat Transfer Coefficient (w/m2-k) 0

Free Stream Temperature (k) 300

Wall Motion 0

Shear Boundary Condition 0

Define wall motion relative to adjacent cell zone? yes

Apply a rotational velocity to this wall? no

Velocity Magnitude $(\mathrm{m} / \mathrm{s})$

$\mathrm{X}$-Component of Wall Translation

Y-Component of Wall Translation 0

Z-Component of Wall Translation 0

Define wall velocity components? no

$\mathrm{X}$-Component of Wall Translation (m/s) 0

Y-Component of Wall Translation $(\mathrm{m} / \mathrm{s}) \quad 0$

Z-Component of Wall Translation (m/s) 0

External Emissivity 1

External Radiation Temperature (k) 300

Wall Roughness Height (m) 0

Wall Roughness Constant 0.5

Rotation Speed ( $\mathrm{rad} / \mathrm{s})$

$\mathrm{X}$-Position of Rotation-Axis Origin (m) 0

Y-Position of Rotation-Axis Origin (m)

Z-Position of Rotation-Axis Origin (m) 0

$\mathrm{X}$-Component of Rotation-Axis Direction 0

Y-Component of Rotation-Axis Direction 0

Z-Component of Rotation-Axis Direction 1

$\mathrm{X}$-component of shear stress (pascal) 0

Y-component of shear stress (pascal) 0

Z-component of shear stress (pascal) 0 
Surface tension gradient $(\mathrm{n} / \mathrm{m}-\mathrm{k})$

0

Specularity Coefficient

outlet

\begin{tabular}{ll} 
Condition & Value \\
\hline Gauge Pressure (pascal) & 0 \\
Backflow Total Temperature (k) & 300 \\
Backflow Direction Specification Method & 2 \\
Coordinate System & 0 \\
X-Component of Flow Direction & 1 \\
Y-Component of Flow Direction & 0 \\
Z-Component of Flow Direction & 0 \\
X-Component of Axis Direction & 1 \\
Y-Component of Axis Direction & 0 \\
Z-Component of Axis Direction & 0 \\
X-Coordinate of Axis Origin (m) & 0 \\
Y-Coordinate of Axis Origin (m) & 0 \\
Z-Coordinate of Axis Origin (m) & 0 \\
Turbulent Specification Method & 0 \\
Backflow Turbulent Kinetic Energy (m2/s2) & 0.7 \\
Backflow Specific Dissipation Rate (1/s) & 1 \\
Backflow Turbulent Intensity (\%) & 10 \\
Backflow Turbulent Length Scale (m) & 1 \\
Backflow Hydraulic Diameter (m) & 1 \\
Backflow Turbulent Viscosity Ratio & 10 \\
is zone used in mixing-plane model? & no \\
Radial Equilibrium Pressure Distribution & 5000000 \\
Specify targeted mass flow rate & 1 \\
Targeted mass flow (kg/s) & no \\
Upper Limit of Absolute Pressure Value (pascal) \\
Lower Limit of Absolute Pressure Value (pascal) \\
\end{tabular}

rightbuilding

\begin{tabular}{|c|c|}
\hline Condition & Value \\
\hline Wall Thickness (m) & 0 \\
\hline Heat Generation Rate (w/m3) & 0 \\
\hline Material Name & aluminum \\
\hline Thermal BC Type & 1 \\
\hline Temperature (k) & 300 \\
\hline Heat Flux $(w / m 2)$ & 0 \\
\hline Convective Heat Transfer Coefficient (w/m2-k) & 0 \\
\hline Free Stream Temperature (k) & 300 \\
\hline Wall Motion & 0 \\
\hline Shear Boundary Condition & 0 \\
\hline Define wall motion relative to adjacent cell zone? & yes \\
\hline Apply a rotational velocity to this wall? & no \\
\hline Velocity Magnitude $(\mathrm{m} / \mathrm{s})$ & 0 \\
\hline X-Component of Wall Translation & 1 \\
\hline Y-Component of Wall Translation & 0 \\
\hline Z-Component of Wall Translation & 0 \\
\hline Define wall velocity components? & no \\
\hline $\mathrm{X}$-Component of Wall Translation $(\mathrm{m} / \mathrm{s})$ & 0 \\
\hline Y-Component of Wall Translation $(\mathrm{m} / \mathrm{s})$ & 0 \\
\hline Z-Component of Wall Translation $(\mathrm{m} / \mathrm{s})$ & 0 \\
\hline External Emissivity & 1 \\
\hline External Radiation Temperature (k) & 300 \\
\hline Wall Roughness Height (m) & 0 \\
\hline Wall Roughness Constant & 0.5 \\
\hline
\end{tabular}


Rotation Speed ( $\mathrm{rad} / \mathrm{s}$ )

$\mathrm{X}$-Position of Rotation-Axis Origin (m)

Y-Position of Rotation-Axis Origin (m)

Z-Position of Rotation-Axis Origin (m)

$\mathrm{X}$-Component of Rotation-Axis Direction

$\mathrm{Y}$-Component of Rotation-Axis Direction

Z-Component of Rotation-Axis Direction

$\mathrm{X}$-component of shear stress (pascal)

Y-component of shear stress (pascal)

Z-component of shear stress (pascal)

Surface tension gradient $(\mathrm{n} / \mathrm{m}-\mathrm{k})$

Specularity Coefficient

ghtbuildingtop

Condition

Value

Wall Thickness (m)

Heat Generation Rate (w/m3)

Material Name

Thermal BC Type

Temperature (k)

Heat Flux (w/m2)

Heat Transfer Coefficient (w/m2-k)

Free Stream Temperature (k) 300

Wall Motion

Shear Boundary Condition

0

Define wall motion relative to adjacent cell zone? yes

Apply a rotational velocity to this wall? no

Velocity Magnitude (m/s)

$\mathrm{X}$-Component of Wall Translation

Y-Component of Wall Translation

Z-Component of Wall Translation

Define wall velocity components?

$\mathrm{X}$-Component of Wall Translation $(\mathrm{m} / \mathrm{s})$

Y-Component of Wall Translation ( $\mathrm{m} / \mathrm{s}$ )

Z-Component of Wall Translation $(\mathrm{m} / \mathrm{s})$

External Emissivity

External Radiation Temperature (k)

Wall Roughness Height (m)

Wall Roughness Constant

Rotation Speed ( $\mathrm{rad} / \mathrm{s}$ )

$\mathrm{X}$-Position of Rotation-Axis Origin (m)

Y-Position of Rotation-Axis Origin (m)

Z-Position of Rotation-Axis Origin (m)

$\mathrm{X}$-Component of Rotation-Axis Direction

Y-Component of Rotation-Axis Direction

Z-Component of Rotation-Axis Direction

$\mathrm{X}$-component of shear stress (pascal)

Y-component of shear stress (pascal)

Z-component of shear stress (pascal)

Surface tension gradient $(\mathrm{n} / \mathrm{m}-\mathrm{k})$

Specularity Coefficient

\section{0}

aluminum

1

300

(1)

300

(1)

0

1

0

no

0

0

0

300

0.5

0

0

0

0

1

0

0

0

0

symmetry

Condition Value

Solver Settings 


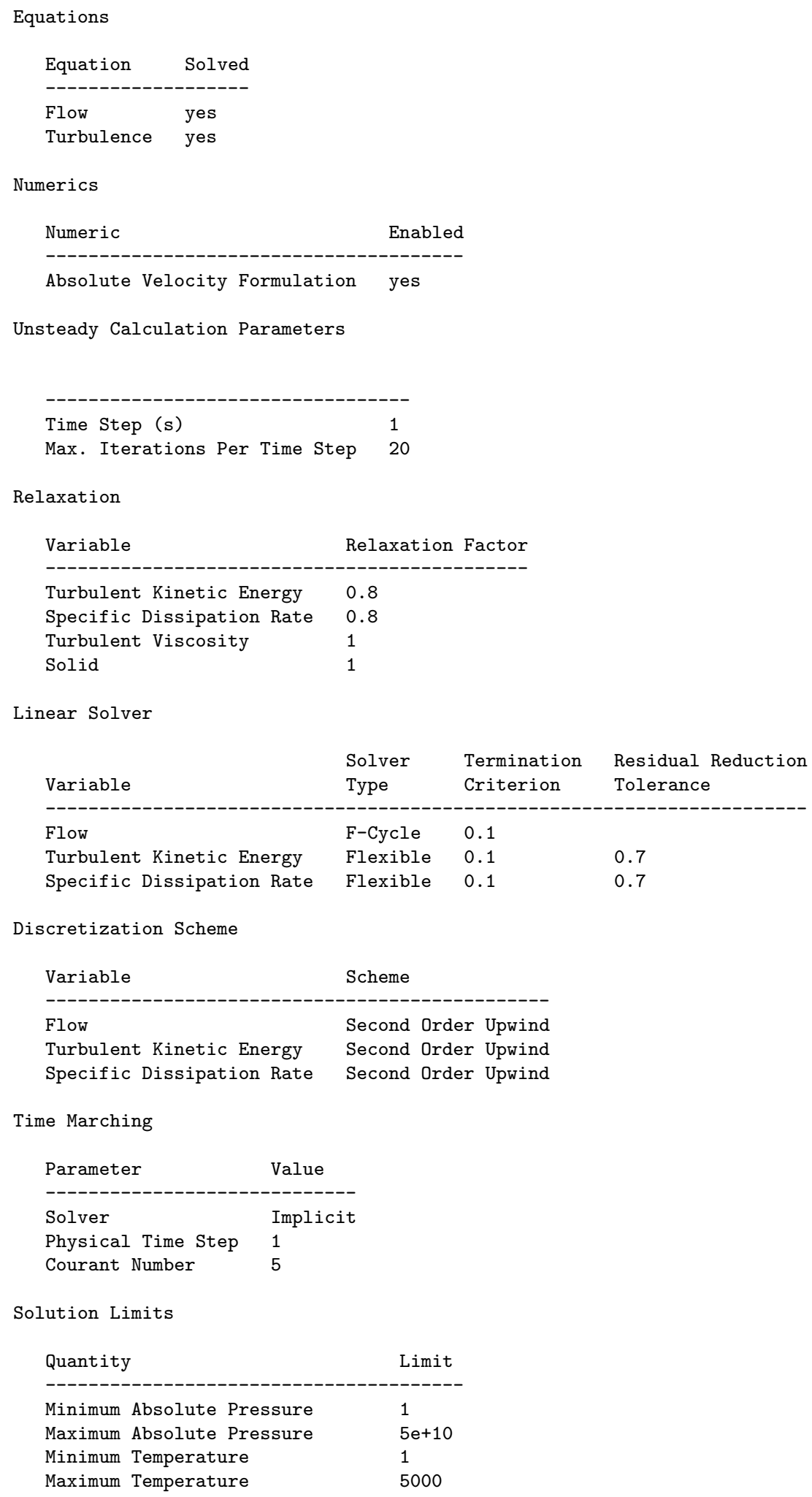


Minimum Turb. Kinetic Energy 1e-14

Minimum Spec. Dissipation Rate $1 \mathrm{e}-20$

Maximum Turb. Viscosity Ratio 100000 



\section{Appendix I}

\section{Sample Output File}

A sample output file of FLUENT's processing for a case along with calculations of the iterations for the first and last time steps.

Cluster: Gup

Welcome to ANSYS FLUENT 12.1.4

Copyright 2009 ANSYS Inc.

All Rights Reserved. Unauthorized use, distribution or duplication is prohibited. ANSYS and FLUENT are trademarks or registered trademarks of ANSYS, Inc. or its subsidiaries in the United States or other countries.

Loading "/opt/sharcnet/ansys/12.1.1/v121/fluent/fluent12.1.4/lib/flprim.dmp.1119-64" Done.

This is a version of FLUENT intended for use by students, academic staff, and faculty. Usage of studentFLUENT is

limited to the terms and conditions specified in the

Clickwrap Software License Agreement for studentFLUENT.

Host spawning Node 0 on machine "gup7" (unix).

/opt/sharcnet/ansys/12.1.1/v121/fluent/fluent12.1.4/bin/fluent -r12.1.4 3ddp -node -alnamd64 -t8 -pinfiniband -mpi=openmpi - cnf=/var/spool/torque/aux//78547.gup-hn.guppy.sharcnet -ssh -mport $10.36 .14 .7: 10.36 \cdot 14.7: 52642: 0$

Starting fixfiledes /opt/sharcnet/ansys/12.1.1/v121/fluent/fluent12.1.4/multiport/mpi/lnamd64/openmpi/bin/ mpirun --app/tmp/fluent-appfile.5984

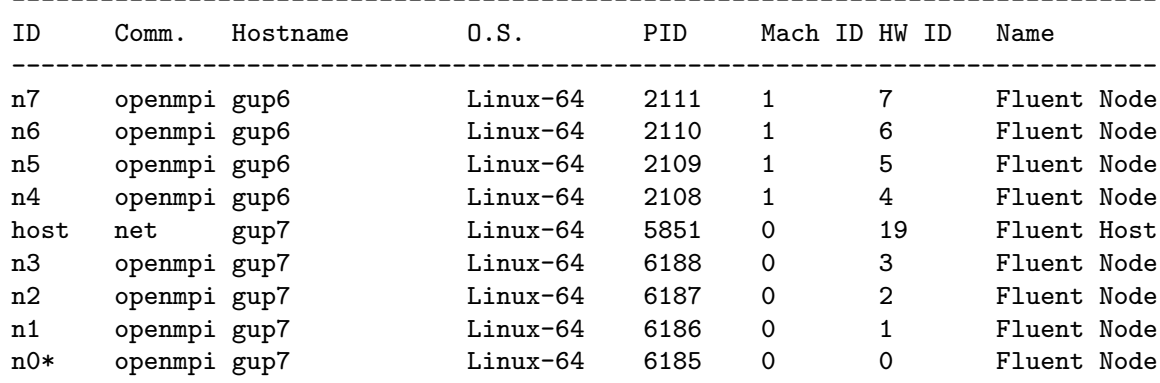


Selected system interconnect: InfiniBand

$>/$ file/set-batch-options no yes no

$>$ /file/read-case /scratch/amacquar/guppy/MeshFiles/FFF.Rt_1_Rx_O_Ry_O_AOA_0.msh

Multicore processors detected. Processor affinity set!

Reading "/scratch/amacquar/guppy/MeshFiles/FFF.Rt_1_Rx_0_Ry_O_AOA_0.msh"... ...........................

419064 nodes, binary.

27651 nodes, binary.

3540180 mixed interior faces, zone 1 , binary.

323 mixed interior faces, zone 2 , binary.

387 mixed interior faces, zone 3 , binary.

13358 mixed wall faces, zone 7 , binary.

1766 triangular velocity-inlet faces, zone 8, binary.

11721 triangular wall faces, zone 9, binary.

1064 triangular wall faces, zone 10, binary.

2324 triangular pressure-outlet faces, zone 11, binary.

11709 triangular wall faces, zone 12, binary.

1022 triangular wall faces, zone 13, binary.

9218 mixed symmetry faces, zone 14, binary.

1667771 mixed cells, zone 4, binary.

Building...

mesh

auto partitioning mesh by Metis (fast),

distributing mesh

parts........,

faces.........,

nodes.........,

cells........,

materials,

Loading material properties database...

interface,

domains,

zones,

symmetry

rightbuildingtop

rightbuilding

outlet

leftbuildingtop

leftbuilding

inlet

ground

model-fluid

turbineout

turbine

interior-model-fluid

parallel,

Done.

$>$ /define/user-defined/compiled-functions compile

/scratch/amacquar/guppy/Rt_1_Rx_0_Ry_O_AOA_O/libudf yes

Give C-Source file names:

/scratch/amacquar/guppy/Rt_1_Rx_0_Ry_0_AOA_0/libudf/abl_udf.c ""

Give header file names:

/scratch/amacquar/guppy/Rt_1_Rx_O_Ry_O_AOA_O/libudf/abl_udf.c " " 
Done.

>/define/user-defined/compiled-functions load/scratch/amacquar/guppy/Rt_3_Rx_0_Ry_0_AOA_0/libudf

Opening library "/scratch/amacquar/guppy/Rt_1_Rx_0_Ry_0_AOA_0/libudf"...

Library "/scratch/amacquar/guppy/Rt_1_Rx_0_Ry_0_AOA_0/libudf/lnamd64/3ddp_host/libudf .so" opened inlet_u_velocity

Done.

Opening library "/scratch/amacquar/guppy/Rt_1_Rx_O_Ry_O_AOA_0/libudf"...

Library "/scratch/amacquar/guppy/Rt_1_Rx_0_Ry_O_AOA_O/libudf/lnamd64/3ddp_node/libudf.so" opened Opening library "/scratch/amacquar/guppy/Rt_1_Rx_O_Ry_O_AOA_O/libudf"...

Library "/scratch/amacquar/guppy/Rt_1_Rx_0_Ry_0_AOA_0/libudf/lnamd64/3ddp_node/libudf.so" opened Opening library "/scratch/amacquar/guppy/Rt_1_Rx_O_Ry_O_AOA_O/libudf"...

Library "/scratch/amacquar/guppy/Rt_1_Rx_0_Ry_O_AOA_O/libudf/lnamd64/3ddp_node/libudf.so" opened Opening library "/scratch/amacquar/guppy/Rt_1_Rx_O_Ry_O_AOA_O/libudf"...

Library "/scratch/amacquar/guppy/Rt_1_Rx_0_Ry_O_AOA_O/libudf/lnamd64/3ddp_node/libudf.so" opened Opening library "/scratch/amacquar/guppy/Rt_1_Rx_O_Ry_O_AOA_O/libudf"...

Library "/scratch/amacquar/guppy/Rt_1_Rx_0_Ry_O_AOA_O/libudf/lnamd64/3ddp_node/libudf.so" opened Opening library "/scratch/amacquar/guppy/Rt_1_Rx_O_Ry_O_AOA_O/libudf"...

Library "/scratch/amacquar/guppy/Rt_1_Rx_0_Ry_O_AOA_O/libudf/lnamd64/3ddp_node/libudf.so" opened Opening library "/scratch/amacquar/guppy/Rt_1_Rx_O_Ry_O_AOA_O/libudf"...

Library "/scratch/amacquar/guppy/Rt_1_Rx_0_Ry_O_AOA_O/libudf/lnamd64/3ddp_node/libudf.so" opened Opening library "/scratch/amacquar/guppy/Rt_1_Rx_O_Ry_O_AOA_O/libudf"...

Library "/scratch/amacquar/guppy/Rt_1_Rx_0_Ry_O_AOA_O/libudf/lnamd64/3ddp_node/libudf.so" opened

$>$ /define/custom-field-functions/define "abl"Enter Custom Field Function:

$4.16 * 1.31 * \log 10\left(\left(z_{-}\right.\right.$coordinate +0.03$\left.) / 0.03\right) / \log 10(60 / 0.03)$

$>$ /define/custom-field-functions/define "power_aug"Enter Custom Field Function: 1/2*density*(x_velocity)^3

$>$ /define/custom-field-functions/define "power_abl"Enter Custom Field Function: 1/2*density*(abl)^3

$>$ /define/custom-field-functions/define "initial_u_velocity"Enter Custom Field Function: abl*cos(0*PI/180)

$>$ /define/custom-field-functions/define "initial_v_velocity"Enter Custom Field Function: abl*sin(0*PI/180)

$>/$ define/models/solver/density-based-implicit yes

$>/$ define/models/energy yes

$>/$ define/models/viscous kw-sst yes

$>$ /define/boundary-conditions/velocity-inlet (inlet)

inlet no no yes yes yes yes profile names list: ("udf")

"udf"data names list: ("inlet_u_velocity: :libudf")

"inlet_u_velocity::libudf" no 300 no yes 101 no 0

$>$ /define/boundary-conditions/pressure-outlet (outlet)

outlet no 0 no 300 no no yes yes no 0.7 no 1 no no

$>$ /define/boundary-conditions/wall (ground leftbuilding leftbuildingtop rightbuilding rightbuildingtop) ground 0 no 0 no no no 0 no no no 0.03 no 0.5

$>/$ define/boundary-conditions/wall (ground leftbuilding leftbuildingtop rightbuilding rightbuildingtop) leftbuilding 0 no 0 no no no 0 no no no 0 no 0.5

$>$ /define/boundary-conditions/copy-bc leftbuilding rightbuilding leftbuildingtop rightbuildingtop ()Copy leftbuilding boundary conditions to rightbuildingtop

Copy leftbuilding boundary conditions to leftbuildingtop

Copy leftbuilding boundary conditions to rightbuilding 
$>/$ solve/set/discretization-scheme/amg-c 1

$>$ /solve/set/discretization-scheme/k 1

$>$ /solve/set/discretization-scheme/omega 1

> /solve/set/under-relaxation/k 0.8

>/solve/set/under-relaxation/omega 0.8

> /solve/set/under-relaxation/turb-viscosity 1

>/solve/monitors/residual/criterion-type 1

The requested convergence criterion type is not available

$>$ /solve/monitors/residual monitor yes yes yes yes yes yes yes

$>$ /solve/set/flow-warnings no

>/solve/initialize/set-defaults k 0.7

> /solve/initialize/set-defaults omega 1

$>$ /solve/initialize/set-defaults temperature 300

>/solve/initialize/initialize-flow

>/solve/patch Model-fluid () x-velocity no yes initial_u_velocity

> /solve/patch Model-fluid () y-velocity no yes initial_v_velocity

>/solve/initialize/set-fmg-initialization

Customize your FMG initialization:

5

set FMG parameters on levels ..

residual reduction on level 10.001 number of cycles on level 110

residual reduction on level 20.001 number of cycles on level 250

residual reduction on level 30.001 number of cycles on level 3500

residual reduction on level 40.001 number of cycles on level 4500

residual reduction on level 5 [coarsest grid]0.001 number of cycles on level 5100

Number of FMG (and FAS geometric multigrid) levels: 5

* FMG customization summary:

* residual reduction on level 0 [finest grid] is: 0.001

* number of cycles on level 0 is: 1

* residual reduction on level 1 is: 0.001

* number of cycles on level 1 is: 10

* residual reduction on level 2 is: 0.001

* number of cycles on level 2 is: 50

* residual reduction on level 3 is: 0.001

* number of cycles on level 3 is: 500

* residual reduction on level 4 is: 0.001

* number of cycles on level 4 is: 500

* residual reduction on level 5 [coarsest grid] is: 0.001

* number of cycles on level 5 is: 100

* FMG customization complete

0.75 yes

>/solve/initialize/fmg-initialization yes 


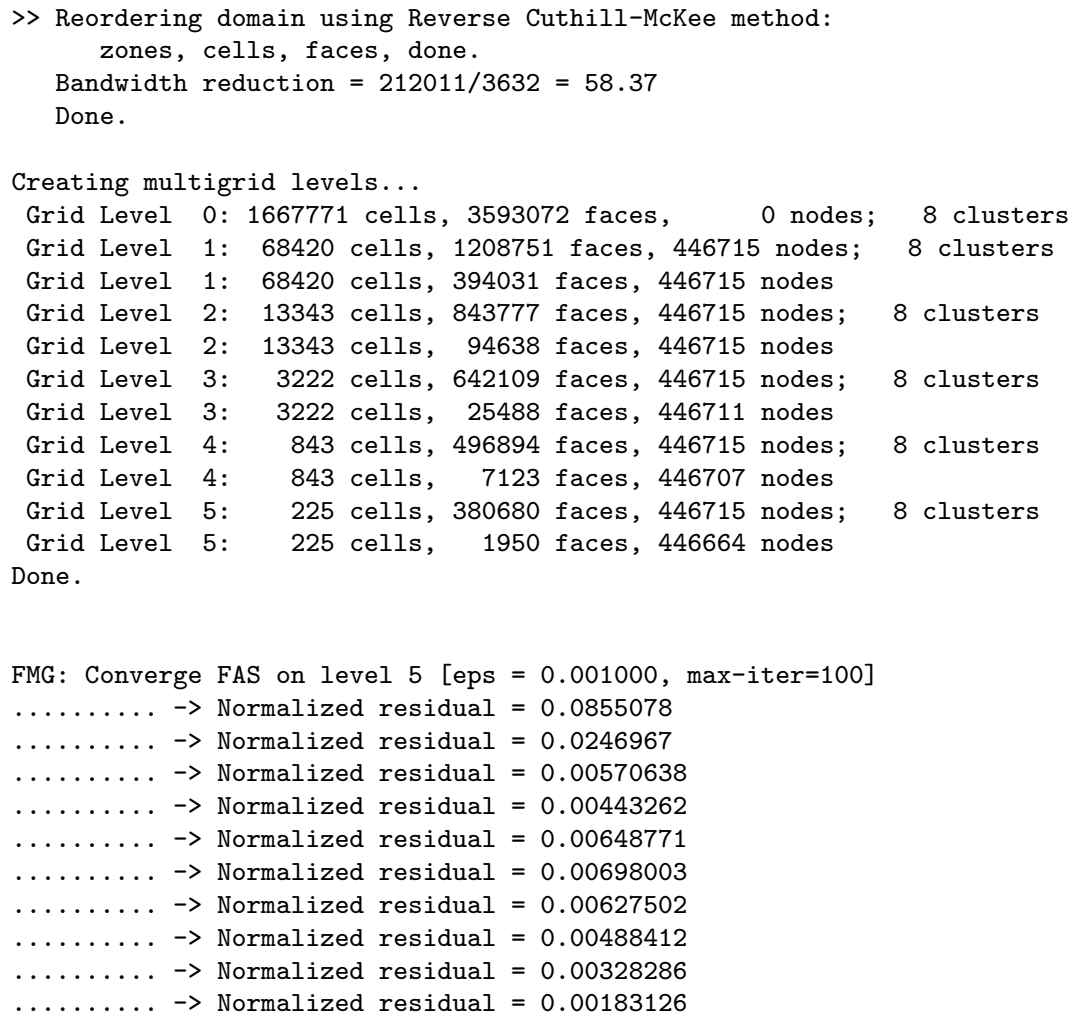

FMG: FAS reached maximum iterations.

Normalized residual $=0.00183126$

FMG: Finished work on level $=5$

FMG: Interpolate solution on next level .. . end

FMG: Converge FAS on level 4 [eps $=0.001000, \max$-iter $=500]$

....... $\rightarrow$ Normalized residual $=0.031736$

........ $\rightarrow$ Normalized residual $=0.00571157$

$\ldots \ldots \ldots$. Normalized residual $=0.00403975$

$\ldots \ldots \ldots$. Normalized residual $=0.00188588$

FMG: FAS converged.

FMG: Finished work on level $=4$

FMG: Interpolate solution on next level ... . end

FMG: Converge FAS on level 3 [eps $=0.001000, \max$-iter $=500$ ] ........ $\rightarrow$ Normalized residual $=0.054006$

$\ldots \ldots \ldots \rightarrow$ Normalized residual $=0.0101748$

$\ldots \ldots \ldots$. Normalized residual $=0.00622941$

$\ldots \ldots \ldots$. . Normalized residual $=0.00342219$

....... $\rightarrow$ Normalized residual $=0.0020179$

$\ldots \ldots \ldots \rightarrow$ Normalized residual $=0.00138015$

$\ldots$

FMG: FAS converged. 
FMG: Finished work on level $=3$

FMG: Interpolate solution on next level .. . end

FMG: Converge FAS on level 2 [eps $=0.001000$, max-iter=50]

$\ldots \ldots \ldots \rightarrow$ Normalized residual $=0.115932$

$\ldots \ldots \ldots$. Normalized residual $=0.024402$

$\ldots \ldots \ldots . . .+$ Normalized residual $=0.0138924$

$\ldots \ldots \ldots . . .$. Normalized residual $=0.0149562$

....... Normalized residual $=0.00540462$

FMG: FAS reached maximum iterations.

Normalized residual $=0.00540462$

FMG: Finished work on level $=2$

FMG: Interpolate solution on next level .. . end

FMG: Converge FAS on level 1 [eps $=0.001000, \max$-iter=10]

$\ldots \ldots \ldots \rightarrow$ Normalized residual $=1.31067$

FMG: FAS reached maximum iterations.

Normalized residual $=1.31067$

FMG: Finished work on level $=1$

FMG: Interpolate solution on next level ... end

$0 .->1 .->2 .,>3 .->4 .->5 .<<<<$

$>$ /solve/iterate 50

iter continuity $\mathrm{x}$-velocity $\mathrm{y}$-velocity $\mathrm{z}$-velocity energy omega time/iter

$\begin{array}{llllllllll}1 & 1.0000 e+00 & 1.0000 e+00 & 1.0000 e+00 & 1.0000 e+00 & 1.0000 e+00 & 3.0235 e-03 & 6.8717 e-02 & 0: 02: 47 & 49\end{array}$

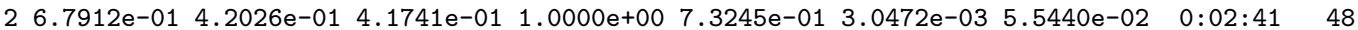

$\begin{array}{llllllllll}3 & 2.9705 e-01 & 2.8333 e-01 & 2.8343 e-01 & 1.0000 e+00 & 3.0660 e-01 & 3.0738 e-03 & 4.9367 e-02 & 0: 02: 36 & 47\end{array}$

$\begin{array}{llllllllll}4 & 1.3025 e-01 & 2.0877 e-01 & 2.0897 e-01 & 1.0000 e+00 & 1.3797 e-01 & 3.0826 e-03 & 4.4259 e-02 & 0: 02: 31 & 46\end{array}$

$\begin{array}{llllllllll}5 & 1.1859 \mathrm{e}-01 & 1.1128 \mathrm{e}-01 & 1.1178 \mathrm{e}-01 & 7.3986 \mathrm{e}-01 & 1.2322 \mathrm{e}-01 & 3.0834 \mathrm{e}-03 & 3.9836 \mathrm{e}-02 & 0: 02: 27 & 45\end{array}$

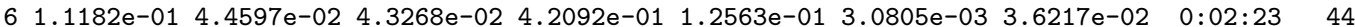

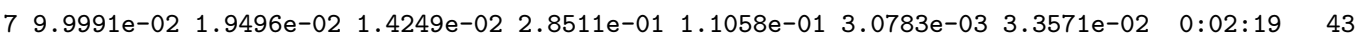

8 8.5705e-02 $1.5012 \mathrm{e}-02 \quad 9.4597 \mathrm{e}-03 \quad 2.4849 \mathrm{e}-01 \quad 9.4347 \mathrm{e}-02 \quad 3.0778 \mathrm{e}-03 \quad 3.1693 \mathrm{e}-02 \quad 0: 02: 15 \quad 42$

$97.3269 \mathrm{e}-02 \quad 1.3348 \mathrm{e}-02 \quad 8.8468 \mathrm{e}-03 \quad 2.4551 \mathrm{e}-01 \quad 7.9407 \mathrm{e}-02 \quad 3.0791 \mathrm{e}-03 \quad 2.9607 \mathrm{e}-02 \quad 0: 02: 11 \quad 41$

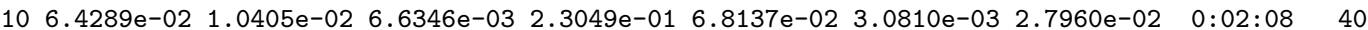

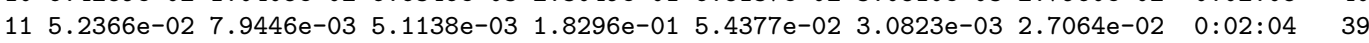

iter continuity $\mathrm{x}$-velocity $\mathrm{y}$-velocity $\mathrm{z}$-velocity $\mathrm{k}$ omega time/iter

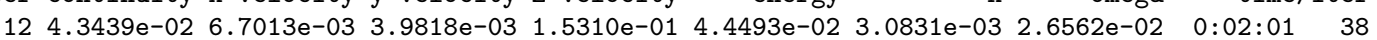

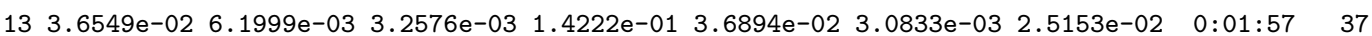

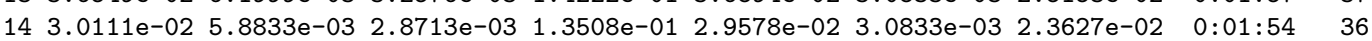

$152.3907 \mathrm{e}-02 \quad 5.4675 \mathrm{e}-03 \quad 2.6960 \mathrm{e}-03 \quad 1.2443 \mathrm{e}-01 \quad 2.2164 \mathrm{e}-02 \quad 3.0831 \mathrm{e}-03 \quad 2.2235 \mathrm{e}-02 \quad 0: 01: 51 \quad 35$

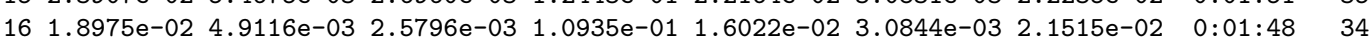

$171.7249 \mathrm{e}-02 \quad 4.2337 \mathrm{e}-03 \quad 2.4264 \mathrm{e}-03 \quad 9.2835 \mathrm{e}-02 \quad 1.4402 \mathrm{e}-02 \quad 3.0881 \mathrm{e}-03 \quad 2.0987 \mathrm{e}-02 \quad 0: 01: 44 \quad 33$

$181.8976 \mathrm{e}-02 \quad 3.5788 \mathrm{e}-03 \quad 2.1787 \mathrm{e}-03 \quad 7.8994 \mathrm{e}-02 \quad 1.7637 \mathrm{e}-02 \quad 3.0938 \mathrm{e}-03 \quad 2.0475 \mathrm{e}-02 \quad 0: 01: 41 \quad 32$

$192.1914 \mathrm{e}-02 \quad 3.0765 \mathrm{e}-03 \quad 1.8024 \mathrm{e}-03 \quad 7.0451 \mathrm{e}-02 \quad 2.1797 \mathrm{e}-02 \quad 3.1027 \mathrm{e}-03 \quad 1.9977 \mathrm{e}-02 \quad 0: 01: 38 \quad 31$

$202.4315 \mathrm{e}-02 \quad 2.7396 \mathrm{e}-03 \quad 1.4048 \mathrm{e}-03 \quad 6.5945 \mathrm{e}-02 \quad 2.4632 \mathrm{e}-02 \quad 3.1158 \mathrm{e}-03 \quad 1.9386 \mathrm{e}-02 \quad 0: 01: 35 \quad 30$

$212.5889 \mathrm{e}-02 \quad 2.5306 \mathrm{e}-03 \quad 1.1076 \mathrm{e}-03 \quad 6.2770 \mathrm{e}-02 \quad 2.5899 \mathrm{e}-02 \quad 3.1348 \mathrm{e}-03 \quad 1.8571 \mathrm{e}-02 \quad 0: 01: 32 \quad 29$

$\begin{array}{llllllllll}22 & 2.7448 \mathrm{e}-02 & 2.2556 \mathrm{e}-03 & 9.2190 \mathrm{e}-04 & 5.8480 \mathrm{e}-02 & 2.6575 \mathrm{e}-02 & 3.1617 \mathrm{e}-03 & 1.7784 \mathrm{e}-02 & 0: 01: 28 & 28\end{array}$

iter continuity $\mathrm{x}$-velocity y-velocity z-velocity energy $\mathrm{k}$ omega time/iter

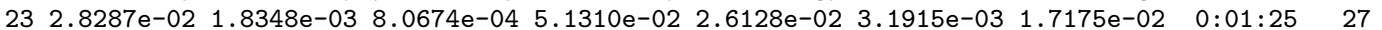

$242.6370 \mathrm{e}-02 \quad 1.4351 \mathrm{e}-03 \quad 5.7988 \mathrm{e}-04 \quad 4.1284 \mathrm{e}-02 \quad 2.3435 \mathrm{e}-02 \quad 3.2276 \mathrm{e}-03 \quad 1.6525 \mathrm{e}-02 \quad 0: 01: 22 \quad 26$

$\begin{array}{llllllllll}25 & 2.2826 e-02 & 1.0334 \mathrm{e}-03 & 3.2453 \mathrm{e}-04 & 2.8205 e-02 & 1.9707 \mathrm{e}-02 & 3.2643 e-03 & 1.6024 \mathrm{e}-02 & 0: 01: 19 & 25\end{array}$

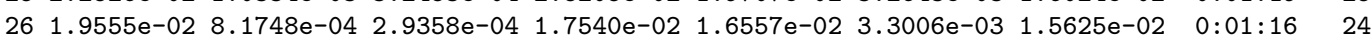


$271.7178 \mathrm{e}-02 \quad 7.8342 \mathrm{e}-04 \quad 3.6729 \mathrm{e}-04 \quad 1.4107 \mathrm{e}-02 \quad 1.4356 \mathrm{e}-02 \quad 3.3283 \mathrm{e}-03 \quad 1.5215 \mathrm{e}-02$ $281.5753 \mathrm{e}-02 \quad 7.5118 \mathrm{e}-04 \quad 3.8920 \mathrm{e}-04 \quad 1.4843 \mathrm{e}-02 \quad 1.3059 \mathrm{e}-02 \quad 3.3384 \mathrm{e}-03 \quad 1.4839 \mathrm{e}-02$ $291.5072 \mathrm{e}-02 \quad 6.7562 \mathrm{e}-04 \quad 3.6519 \mathrm{e}-04 \quad 1.5238 \mathrm{e}-02 \quad 1.2454 \mathrm{e}-02 \quad 3.3460 \mathrm{e}-03 \quad 1.4516 \mathrm{e}-02$ $301.4902 \mathrm{e}-02 \quad 5.7821 \mathrm{e}-04 \quad 3.1427 \mathrm{e}-04 \quad 1.5030 \mathrm{e}-02 \quad 1.2320 \mathrm{e}-02 \quad 3.3482 \mathrm{e}-03 \quad 1.4210 \mathrm{e}-02$ $31 \quad 1.5012 \mathrm{e}-02 \quad 5.0188 \mathrm{e}-04 \quad 2.6311 \mathrm{e}-04 \quad 1.4250 \mathrm{e}-02 \quad 1.2440 \mathrm{e}-02 \quad 3.3373 \mathrm{e}-03 \quad 1.3949 \mathrm{e}-02$ $321.5238 \mathrm{e}-02 \quad 4.6158 \mathrm{e}-04 \quad 2.2707 \mathrm{e}-04 \quad 1.3269 \mathrm{e}-02 \quad 1.2664 \mathrm{e}-02 \quad 3.3258 \mathrm{e}-03 \quad 1.3749 \mathrm{e}-02$ 33 1.5362e-02 4.4433e-04 1.9920e-04 1.2389e-02 $1.2808 \mathrm{e}-02 \quad 3.3106 \mathrm{e}-03 \quad 1.3621 \mathrm{e}-02$ iter continuity $\mathrm{x}$-velocity $\mathrm{y}$-velocity $\mathrm{z}$-velocity energy $\mathrm{k}$ omega $341.5431 \mathrm{e}-02 \quad 4.4539 \mathrm{e}-04 \quad 1.7822 \mathrm{e}-04 \quad 1.1756 \mathrm{e}-02 \quad 1.2905 \mathrm{e}-02 \quad 3.2879 \mathrm{e}-03 \quad 1.3522 \mathrm{e}-02$ $351.5488 \mathrm{e}-02 \quad 4.4915 \mathrm{e}-04 \quad 1.6048 \mathrm{e}-04 \quad 1.1359 \mathrm{e}-02 \quad 1.2987 \mathrm{e}-02 \quad 3.2706 \mathrm{e}-03 \quad 1.3382 \mathrm{e}-02$ $361.5542 \mathrm{e}-02 \quad 4.5035 \mathrm{e}-04 \quad 1.4599 \mathrm{e}-04 \quad 1.1055 \mathrm{e}-02 \quad 1.3059 \mathrm{e}-02 \quad 3.2440 \mathrm{e}-03 \quad 1.3215 \mathrm{e}-02$ $37 \quad 1.5506 \mathrm{e}-02 \quad 4.3891 \mathrm{e}-04 \quad 1.3626 \mathrm{e}-04 \quad 1.0633 \mathrm{e}-02 \quad 1.3045 \mathrm{e}-02 \quad 3.2227 \mathrm{e}-03 \quad 1.3019 \mathrm{e}-02$ $381.5388 \mathrm{e}-02 \quad 4.1922 \mathrm{e}-04 \quad 1.2910 \mathrm{e}-04 \quad 9.9980 \mathrm{e}-03 \quad 1.2952 \mathrm{e}-02 \quad 3.1977 \mathrm{e}-03 \quad 1.2814 \mathrm{e}-02$ $391.5200 \mathrm{e}-02 \quad 3.9423 \mathrm{e}-04 \quad 1.2336 \mathrm{e}-04 \quad 9.2670 \mathrm{e}-03 \quad 1.2792 \mathrm{e}-02 \quad 3.1775 \mathrm{e}-03 \quad 1.2575 \mathrm{e}-02$ $401.4967 \mathrm{e}-02 \quad 3.6702 \mathrm{e}-04 \quad 1.1871 \mathrm{e}-04 \quad 8.5283 \mathrm{e}-03 \quad 1.2593 \mathrm{e}-02 \quad 3.1588 \mathrm{e}-03 \quad 1.2330 \mathrm{e}-02$ $41 \quad 1.4703 \mathrm{e}-02 \quad 3.4151 \mathrm{e}-04 \quad 1.1446 \mathrm{e}-04 \quad 7.9066 \mathrm{e}-03 \quad 1.2370 \mathrm{e}-02 \quad 3.1375 \mathrm{e}-03 \quad 1.2074 \mathrm{e}-02$ $421.4439 \mathrm{e}-02 \quad 3.2263 \mathrm{e}-04 \quad 1.1050 \mathrm{e}-04 \quad 7.4215 \mathrm{e}-03 \quad 1.2149 \mathrm{e}-02 \quad 3.1180 \mathrm{e}-03 \quad 1.1818 \mathrm{e}-02$ $431.4107 \mathrm{e}-02 \quad 3.0833 \mathrm{e}-04 \quad 1.0713 \mathrm{e}-04 \quad 7.0246 \mathrm{e}-03 \quad 1.1868 \mathrm{e}-02 \quad 3.0967 \mathrm{e}-03 \quad 1.1575 \mathrm{e}-02$ $441.3705 e-02 \quad 2.9682 \mathrm{e}-04 \quad 1.0447 \mathrm{e}-04 \quad 6.6753 \mathrm{e}-03 \quad 1.1518 \mathrm{e}-02 \quad 3.0780 \mathrm{e}-03 \quad 1.1357 \mathrm{e}-02$ iter continuity $\mathrm{x}$-velocity $\mathrm{y}$-velocity $\mathrm{z}$-velocity energy $\mathrm{k}$ omega $451.3225 e-02 \quad 2.8510 e-04 \quad 1.0226 e-04 \quad 6.3112 e-03 \quad 1.1084 e-02 \quad 3.0603 e-03 \quad 1.1160 e-02$

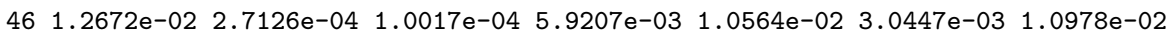
$471.2105 \mathrm{e}-02 \quad 2.5521 \mathrm{e}-04 \quad 9.7985 \mathrm{e}-05 \quad 5.4943 \mathrm{e}-03 \quad 1.0023 \mathrm{e}-02 \quad 3.0292 \mathrm{e}-03 \quad 1.0805 \mathrm{e}-02$ $481.1590 \mathrm{e}-02 \quad 2.3748 \mathrm{e}-04 \quad 9.5424 \mathrm{e}-05 \quad 5.0659 \mathrm{e}-03 \quad 9.5343 \mathrm{e}-03 \quad 3.0158 \mathrm{e}-03 \quad 1.0634 \mathrm{e}-02$ $491.1181 \mathrm{e}-02 \quad 2.2073 \mathrm{e}-04 \quad 9.2462 \mathrm{e}-05 \quad 4.6594 \mathrm{e}-03 \quad 9.1585 \mathrm{e}-03 \quad 3.0039 \mathrm{e}-03 \quad 1.0461 \mathrm{e}-02$ $501.0903 e-02 \quad 2.0646 e-04 \quad 8.9502 e-05 \quad 4.3164 e-03 \quad 8.9199 e-03 \quad 2.9926 e-03 \quad 1.0288 e-02$

$0: 01: 13$ $0: 01: 09$ $0: 01: 06$ $0: 01: 03$ $0: 01: 00$ $0: 00: 57$ $0: 00: 54$ time/iter $0: 00: 50 \quad 16$ $0: 00: 47 \quad 15$ $0: 00: 44 \quad 14$ $0: 00: 41 \quad 13$ $0: 00: 38 \quad 12$ $0: 00: 35 \quad 11$ $0: 00: 31 \quad 10$ $0: 00: 28 \quad 9$ $0: 00: 25 \quad 8$ $0: 00: 22 \quad 7$ $0: 00: 19$ time/iter $0: 00: 16 \quad 5$ $0: 00: 13 \quad 4$ $0: 00: 09$ $0: 00: 06 \quad 2$ $0: 00: 03 \quad 1$ $0: 00: 00$ 18 17 (1) 3 2

9

8

7

6

5
3
2
1
0

$>$ /define/boundary-conditions/modify-zones zone-type turbine porous-jump

$>$ /define/boundary-conditions/porous-jump (turbine)

turbine $1 \mathrm{e}+10 \quad 0.0750 .4$

$>$ /solve/iterate 100

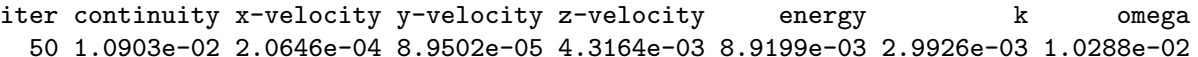
$\begin{array}{llllllll}50 & 1.0903 e-02 & 2.0646 e-04 & 8.9502 e-05 & 4.3164 e-03 & 8.9199 e-03 & 2.9926 e-03 & 1.0288 e-02 \\ 51 & 1.0738 \mathrm{e}-02 & 1.9542 \mathrm{e}-04 & 8.6704 \mathrm{e}-05 & 4.0412 \mathrm{e}-03 & 8.7955 \mathrm{e}-03 & 2.9818 \mathrm{e}-03 & 1.0111 \mathrm{e}-02\end{array}$

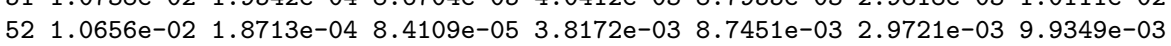
$531.0640 \mathrm{e}-02 \quad 1.8063 \mathrm{e}-04 \quad 8.1919 \mathrm{e}-05 \quad 3.6313 \mathrm{e}-03 \quad 8.7449 \mathrm{e}-03 \quad 2.9630 \mathrm{e}-03 \quad 9.7594 \mathrm{e}-03$ $54 \quad 1.0666 \mathrm{e}-02 \quad 1.7521 \mathrm{e}-04 \quad 8.0030 \mathrm{e}-05 \quad 3.4730 \mathrm{e}-03 \quad 8.7700 \mathrm{e}-03 \quad 2.9548 \mathrm{e}-03 \quad 9.5960 \mathrm{e}-03$ $551.0717 \mathrm{e}-02 \quad 1.7014 \mathrm{e}-04 \quad 7.8341 \mathrm{e}-05 \quad 3.3275 \mathrm{e}-03 \quad 8.8087 \mathrm{e}-03 \quad 2.9476 \mathrm{e}-03 \quad 9.4481 \mathrm{e}-03$ $56 \quad 1.0764 \mathrm{e}-02 \quad 1.6527 \mathrm{e}-04 \quad 7.6763 \mathrm{e}-05 \quad 3.1884 \mathrm{e}-03 \quad 8.8388 \mathrm{e}-03 \quad 2.9407 \mathrm{e}-03 \quad 9.3103 \mathrm{e}-03$ $57 \quad 1.0764 \mathrm{e}-02 \quad 1.6025 \mathrm{e}-04 \quad 7.5114 \mathrm{e}-05 \quad 3.0602 \mathrm{e}-03 \quad 8.8281 \mathrm{e}-03 \quad 2.9347 \mathrm{e}-03 \quad 9.1810 \mathrm{e}-03$ $\begin{array}{llllllll}58 & 1.0735 e-02 & 1.5518 \mathrm{e}-04 & 7.3667 e-05 & 2.9467 e-03 & 8.7964 e-03 & 2.9294 e-03 & 9.0559 e-03\end{array}$ $591.0688 \mathrm{e}-02 \quad 1.5051 \mathrm{e}-04 \quad 7.2405 \mathrm{e}-05 \quad 2.8490 \mathrm{e}-03 \quad 8.7518 \mathrm{e}-03 \quad 2.9241 \mathrm{e}-03 \quad 8.9356 \mathrm{e}-03$ $601.0618 \mathrm{e}-02 \quad 1.4613 \mathrm{e}-04 \quad 7.1307 \mathrm{e}-05 \quad 2.7713 \mathrm{e}-03 \quad 8.6899 \mathrm{e}-03 \quad 2.9189 \mathrm{e}-03 \quad 8.8206 \mathrm{e}-03$ iter continuity $\mathrm{x}$-velocity $\mathrm{y}$-velocity $\mathrm{z}$-velocity $\mathrm{k}$ omega $61 \quad 1.0530 \mathrm{e}-02 \quad 1.4242 \mathrm{e}-04 \quad 7.0266 \mathrm{e}-05 \quad 2.7129 \mathrm{e}-03 \quad 8.6157 \mathrm{e}-03 \quad 2.9140 \mathrm{e}-03 \quad 8.7085 \mathrm{e}-03$ $621.0421 \mathrm{e}-02 \quad 1.3932 \mathrm{e}-04 \quad 6.9128 \mathrm{e}-05 \quad 2.6680 \mathrm{e}-03 \quad 8.5239 \mathrm{e}-03 \quad 2.9091 \mathrm{e}-03 \quad 8.6002 \mathrm{e}-03$ $631.0306 \mathrm{e}-02 \quad 1.3675 \mathrm{e}-04 \quad 6.7901 \mathrm{e}-05 \quad 2.6346 \mathrm{e}-03 \quad 8.4293 \mathrm{e}-03 \quad 2.9044 \mathrm{e}-03 \quad 8.4961 \mathrm{e}-03$ $641.0195 \mathrm{e}-02 \quad 1.3483 \mathrm{e}-04 \quad 6.6541 \mathrm{e}-05 \quad 2.6114 \mathrm{e}-03 \quad 8.3375 \mathrm{e}-03 \quad 2.9000 \mathrm{e}-03 \quad 8.3949 \mathrm{e}-03$ $\begin{array}{llllllll}65 & 1.0104 e-02 & 1.3338 e-04 & 6.5063 e-05 & 2.5927 e-03 & 8.2632 e-03 & 2.8960 e-03 & 8.2999 e-03\end{array}$ $661.0035 \mathrm{e}-02 \quad 1.3234 \mathrm{e}-04 \quad 6.3518 \mathrm{e}-05 \quad 2.5756 \mathrm{e}-03 \quad 8.2070 \mathrm{e}-03 \quad 2.8923 \mathrm{e}-03 \quad 8.2087 \mathrm{e}-03$ $679.9826 \mathrm{e}-03 \quad 1.3150 \mathrm{e}-04 \quad 6.1927 \mathrm{e}-05 \quad 2.5572 \mathrm{e}-03 \quad 8.1641 \mathrm{e}-03 \quad 2.8890 \mathrm{e}-03 \quad 8.1197 \mathrm{e}-03$ $689.9417 e-03 \quad 1.3070 e-04 \quad 6.0305 e-05 \quad 2.5328 e-03 \quad 8.1308 e-03 \quad 2.8861 e-03 \quad 8.0307 e-03$ $699.9115 e-03 \quad 1.2988 e-04 \quad 5.8727 e-05 \quad 2.5000 e-03 \quad 8.1062 e-03 \quad 2.8836 e-03 \quad 7.9414 e-03$ $709.8880 \mathrm{e}-03 \quad 1.2893 \mathrm{e}-04 \quad 5.7246 \mathrm{e}-05 \quad 2.4581 \mathrm{e}-03 \quad 8.0869 \mathrm{e}-03 \quad 2.8814 \mathrm{e}-03 \quad 7.8521 \mathrm{e}-03$

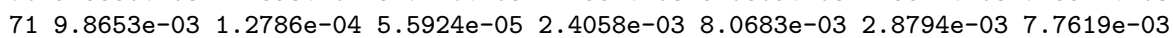
iter continuity $\mathrm{x}$-velocity $\mathrm{y}$-velocity $\mathrm{z}$-velocity energy $\mathrm{k}$ omega

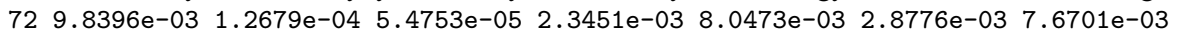
$\begin{array}{llllllll}739.8085 e-03 & 1.2562 e-04 & 5.3713 e-05 & 2.2770 e-03 & 8.0219 e-03 & 2.8761 e-03 & 7.5769 e-03\end{array}$

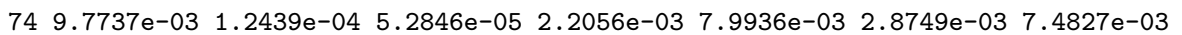
$\begin{array}{llllllll}75 & 9.7373 e-03 & 1.2322 e-04 & 5.2138 e-05 & 2.1326 e-03 & 7.9639 e-03 & 2.8739 e-03 & 7.3875 e-03\end{array}$

time/iter 0:05:15 100 $0: 05: 17 \quad 99$ $0: 05: 1298$ $0: 05: 08 \quad 97$ $0: 05: 0596$ $0: 05: 0195$ $0: 04: 57 \quad 94$ $0: 04: 54 \quad 93$ $0: 04: 51 \quad 92$ $0: 04: 47 \quad 91$ $0: 04: 4490$ time/iter $0: 04: 41 \quad 89$ $0: 04: 37 \quad 88$ $0: 04: 34 \quad 87$ $0: 04: 31 \quad 86$ $0: 04: 28 \quad 85$ $0: 04: 25 \quad 84$ $0: 04: 22 \quad 83$ $0: 04: 18 \quad 82$ $0: 04: 15 \quad 81$ $0: 04: 12 \quad 80$ 0:04:09 79 time/iter 0:04:06 78 $0: 04: 03 \quad 77$ $0: 03: 5976$ $0: 03: 5675$ 
$769.7013 e-03 \quad 1.2214 \mathrm{e}-04 \quad 5.1560 \mathrm{e}-05 \quad 2.0588 \mathrm{e}-03 \quad 7.9346 \mathrm{e}-03 \quad 2.8731 \mathrm{e}-03 \quad 7.2904 \mathrm{e}-03$ $779.6615 \mathrm{e}-03 \quad 1.2117 \mathrm{e}-04 \quad 5.1076 \mathrm{e}-05 \quad 1.9869 \mathrm{e}-03 \quad 7.9023 e-03 \quad 2.8726 \mathrm{e}-03 \quad 7.1909 e-03$ $789.6225 e-03 \quad 1.2036 e-04 \quad 5.0672 e-05 \quad 1.9183 e-03 \quad 7.8705 e-03 \quad 2.8724 e-03 \quad 7.0912 e-03$ $799.5838 \mathrm{e}-03 \quad 1.1970 \mathrm{e}-04 \quad 5.0270 \mathrm{e}-05 \quad 1.8538 \mathrm{e}-03 \quad 7.8390 \mathrm{e}-03 \quad 2.8724 \mathrm{e}-03 \quad 6.9914 \mathrm{e}-03$ $809.5488 \mathrm{e}-03 \quad 1.1920 \mathrm{e}-04 \quad 4.9894 \mathrm{e}-05 \quad 1.7957 \mathrm{e}-03 \quad 7.8105 e-03 \quad 2.8727 e-03 \quad 6.8917 \mathrm{e}-03$ $819.5197 e-03 \quad 1.1891 e-04 \quad 4.9508 e-05 \quad 1.7445 e-03 \quad 7.7868 e-03 \quad 2.8733 e-03 \quad 6.7919 e-03$ $829.4989 e-03 \quad 1.1881 e-04 \quad 4.9114 e-05 \quad 1.7005 e-03 \quad 7.7699 e-03 \quad 2.8740 e-03 \quad 6.6914 e-03$ iter continuity $\mathrm{x}$-velocity $\mathrm{y}$-velocity $\mathrm{z}$-velocity energy $\mathrm{k}$ omega $839.4877 \mathrm{e}-03 \quad 1.1886 \mathrm{e}-04 \quad 4.8712 \mathrm{e}-05 \quad 1.6635 \mathrm{e}-03 \quad 7.7609 \mathrm{e}-03 \quad 2.8749 \mathrm{e}-03 \quad 6.5915 \mathrm{e}-03$ $849.4818 \mathrm{e}-03 \quad 1.1905 \mathrm{e}-04 \quad 4.8284 \mathrm{e}-05 \quad 1.6319 \mathrm{e}-03 \quad 7.7562 \mathrm{e}-03 \quad 2.8759 \mathrm{e}-03 \quad 6.4921 \mathrm{e}-03$ $859.4799 \mathrm{e}-03 \quad 1.1934 \mathrm{e}-04 \quad 4.7836 \mathrm{e}-05 \quad 1.6054 \mathrm{e}-03 \quad 7.7547 \mathrm{e}-03 \quad 2.8771 \mathrm{e}-03 \quad 6.3929 \mathrm{e}-03$ $869.4790 e-03 \quad 1.1967 e-04 \quad 4.7357 e-05 \quad 1.5840 e-03 \quad 7.7540 e-03 \quad 2.8784 e-03 \quad 6.2939 e-03$ $879.4798 \mathrm{e}-03 \quad 1.1999 \mathrm{e}-04 \quad 4.6837 \mathrm{e}-05 \quad 1.5671 \mathrm{e}-03 \quad 7.7548 \mathrm{e}-03 \quad 2.8798 \mathrm{e}-03 \quad 6.1968 \mathrm{e}-03$ $889.4803 e-03 \quad 1.2020 e-04 \quad 4.6295 e-05 \quad 1.5527 e-03 \quad 7.7552 e-03 \quad 2.8813 e-03 \quad 6.1009 e-03$ $899.4840 \mathrm{e}-03 \quad 1.2031 \mathrm{e}-04 \quad 4.5722 \mathrm{e}-05 \quad 1.5399 \mathrm{e}-03 \quad 7.7584 \mathrm{e}-03 \quad 2.8828 \mathrm{e}-03 \quad 6.0078 \mathrm{e}-03$ $909.4889 e-03 \quad 1.2019 e-04 \quad 4.5148 e-05 \quad 1.5263 e-03 \quad 7.7624 e-03 \quad 2.8844 e-03 \quad 5.9182 e-03$ $919.4923 \mathrm{e}-03 \quad 1.1983 \mathrm{e}-04 \quad 4.4564 \mathrm{e}-05 \quad 1.5118 \mathrm{e}-03 \quad 7.7652 \mathrm{e}-03 \quad 2.8861 \mathrm{e}-03 \quad 5.8313 \mathrm{e}-03$ $929.4927 e-03 \quad 1.1928 e-04 \quad 4.3955 e-05 \quad 1.4976 e-03 \quad 7.7654 e-03 \quad 2.8879 e-03 \quad 5.7479 e-03$ $939.4869 \mathrm{e}-03 \quad 1.1849 \mathrm{e}-04 \quad 4.3362 \mathrm{e}-05 \quad 1.4828 \mathrm{e}-03 \quad 7.7607 \mathrm{e}-03 \quad 2.8896 \mathrm{e}-03 \quad 5.6684 \mathrm{e}-03$ iter continuity $\mathrm{x}$-velocity y-velocity $\mathrm{z}$-velocity energy $\mathrm{k}$ omega $949.4751 e-03 \quad 1.1749 e-04 \quad 4.2789 e-05 \quad 1.4683 e-03 \quad 7.7510 e-03 \quad 2.8915 e-03 \quad 5.5910 e-03$ $959.4521 \mathrm{e}-03 \quad 1.1630 \mathrm{e}-04 \quad 4.2240 \mathrm{e}-05 \quad 1.4547 \mathrm{e}-03 \quad 7.7322 \mathrm{e}-03 \quad 2.8934 \mathrm{e}-03 \quad 5.5164 \mathrm{e}-03$ $969.4159 e-03 \quad 1.1494 e-04 \quad 4.1696 e-05 \quad 1.4370 e-03 \quad 7.7025 e-03 \quad 2.8953 e-03 \quad 5.4443 e-03$ $979.3638 \mathrm{e}-03 \quad 1.1342 \mathrm{e}-04 \quad 4.1149 \mathrm{e}-05 \quad 1.4179 \mathrm{e}-03 \quad 7.6598 \mathrm{e}-03 \quad 2.8973 \mathrm{e}-03 \quad 5.3744 \mathrm{e}-03$ $989.2983 e-03 \quad 1.1189 e-04 \quad 4.0597 e-05 \quad 1.4014 e-03 \quad 7.6062 e-03 \quad 2.8993 e-03 \quad 5.3076 e-03$ $999.2191 e-03 \quad 1.1037 e-04 \quad 4.0030 e-05 \quad 1.3866 e-03 \quad 7.5415 e-03 \quad 2.9013 e-03 \quad 5.2436 e-03$ $1009.1281 \mathrm{e}-03 \quad 1.0886 \mathrm{e}-04 \quad 3.9478 \mathrm{e}-05 \quad 1.3724 \mathrm{e}-03 \quad 7.4670 \mathrm{e}-03 \quad 2.9034 \mathrm{e}-03 \quad 5.1829 \mathrm{e}-03$ $1019.0275 \mathrm{e}-03 \quad 1.0744 \mathrm{e}-04 \quad 3.8928 \mathrm{e}-05 \quad 1.3586 \mathrm{e}-03 \quad 7.3845 \mathrm{e}-03 \quad 2.9056 \mathrm{e}-03 \quad 5.1269 \mathrm{e}-03$ $1028.9189 \mathrm{e}-03 \quad 1.0616 \mathrm{e}-04 \quad 3.8350 \mathrm{e}-05 \quad 1.3453 \mathrm{e}-03 \quad 7.2956 \mathrm{e}-03 \quad 2.9078 \mathrm{e}-03 \quad 5.0753 \mathrm{e}-03$ $1038.8086 \mathrm{e}-03 \quad 1.0507 \mathrm{e}-04 \quad 3.7757 \mathrm{e}-05 \quad 1.3318 \mathrm{e}-03 \quad 7.2052 \mathrm{e}-03 \quad 2.9101 \mathrm{e}-03 \quad 5.0287 \mathrm{e}-03$ $1048.7023 e-03 \quad 1.0414 e-04 \quad 3.7158 e-05 \quad 1.3186 e-03 \quad 7.1180 e-03 \quad 2.9125 e-03 \quad 4.9874 e-03$ iter continuity $\mathrm{x}$-velocity $\mathrm{y}$-velocity $\mathrm{z}$-velocity $\mathrm{k}$ omega $1058.6054 \mathrm{e}-03 \quad 1.0342 \mathrm{e}-04 \quad 3.6553 \mathrm{e}-05 \quad 1.3051 \mathrm{e}-03 \quad 7.0385 \mathrm{e}-03 \quad 2.9150 \mathrm{e}-03 \quad 4.9514 \mathrm{e}-03$ $1068.5193 e-03 \quad 1.0286 e-04 \quad 3.5939 e-05 \quad 1.2913 e-03 \quad 6.9679 e-03 \quad 2.9175 e-03 \quad 4.9199 e-03$ $1078.4480 \mathrm{e}-03 \quad 1.0248 \mathrm{e}-04 \quad 3.5325 \mathrm{e}-05 \quad 1.2776 \mathrm{e}-03 \quad 6.9094 \mathrm{e}-03 \quad 2.9201 \mathrm{e}-03 \quad 4.8928 \mathrm{e}-03$ 108 8.3920e-03 $1.0222 \mathrm{e}-04 \quad 3.4731 \mathrm{e}-05 \quad 1.2636 \mathrm{e}-03 \quad 6.8635 \mathrm{e}-03 \quad 2.9227 \mathrm{e}-03 \quad 4.8694 \mathrm{e}-03$ $1098.3547 e-03 \quad 1.0203 e-04 \quad 3.4155 e-05 \quad 1.2497 e-03 \quad 6.8328 e-03 \quad 2.9254 e-03 \quad 4.8504 e-03$ $1108.3367 e-03 \quad 1.0190 e-04 \quad 3.3603 e-05 \quad 1.2363 e-03 \quad 6.8179 e-03 \quad 2.9281 e-03 \quad 4.8345 e-03$ $1118.3380 e-03 \quad 1.0182 e-04 \quad 3.3078 e-05 \quad 1.2227 e-03 \quad 6.8188 e-03 \quad 2.9309 e-03 \quad 4.8211 e-03$ $1128.3529 e-03 \quad 1.0169 e-04 \quad 3.2600 e-05 \quad 1.2088 e-03 \quad 6.8309 e-03 \quad 2.9336 e-03 \quad 4.8094 e-03$ $1138.3802 \mathrm{e}-03 \quad 1.0154 \mathrm{e}-04 \quad 3.2170 \mathrm{e}-05 \quad 1.1952 \mathrm{e}-03 \quad 6.8531 \mathrm{e}-03 \quad 2.9364 \mathrm{e}-03 \quad 4.7981 \mathrm{e}-03$ $1148.4160 e-03 \quad 1.0138 e-04 \quad 3.1769 e-05 \quad 1.1813 e-03 \quad 6.8822 e-03 \quad 2.9392 e-03 \quad 4.7865 e-03$

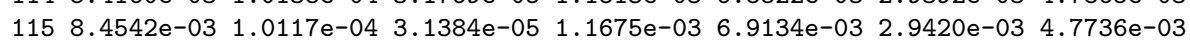
iter continuity $\mathrm{x}$-velocity $\mathrm{y}$-velocity $\mathrm{z}$-velocity $\mathrm{k}$ omega $1168.4924 \mathrm{e}-03 \quad 1.0093 \mathrm{e}-04 \quad 3.1031 \mathrm{e}-05 \quad 1.1536 \mathrm{e}-03 \quad 6.9446 \mathrm{e}-03 \quad 2.9447 \mathrm{e}-03 \quad 4.7587 \mathrm{e}-03$ $1178.5239 e-03 \quad 1.0057 e-04 \quad 3.0713 e-05 \quad 1.1397 e-03 \quad 6.9703 e-03 \quad 2.9473 e-03 \quad 4.7417 e-03$ $1188.5470 \mathrm{e}-03 \quad 1.0016 \mathrm{e}-04 \quad 3.0407 \mathrm{e}-05 \quad 1.1261 \mathrm{e}-03 \quad 6.9892 \mathrm{e}-03 \quad 2.9498 \mathrm{e}-03 \quad 4.7231 \mathrm{e}-03$ $1198.5619 \mathrm{e}-03 \quad 9.9675 \mathrm{e}-05 \quad 3.0104 \mathrm{e}-05 \quad 1.1125 \mathrm{e}-03 \quad 7.0014 \mathrm{e}-03 \quad 2.9521 \mathrm{e}-03 \quad 4.7025 \mathrm{e}-03$ $1208.5658 \mathrm{e}-03 \quad 9.9119 \mathrm{e}-05 \quad 2.9812 \mathrm{e}-05 \quad 1.0987 \mathrm{e}-03 \quad 7.0046 \mathrm{e}-03 \quad 2.9543 \mathrm{e}-03 \quad 4.6801 \mathrm{e}-03$ $1218.5576 e-03 \quad 9.8505 e-05 \quad 2.9520 e-05 \quad 1.0846 e-03 \quad 6.9979 e-03 \quad 2.9563 e-03 \quad 4.6576 e-03$ $1228.5380 \mathrm{e}-03 \quad 9.7863 \mathrm{e}-05 \quad 2.9214 \mathrm{e}-05 \quad 1.0706 \mathrm{e}-03 \quad 6.9818 \mathrm{e}-03 \quad 2.9581 \mathrm{e}-03 \quad 4.6355 \mathrm{e}-03$ $1238.5104 \mathrm{e}-03 \quad 9.7181 \mathrm{e}-05 \quad 2.8900 \mathrm{e}-05 \quad 1.0569 \mathrm{e}-03 \quad 6.9593 \mathrm{e}-03 \quad 2.9596 \mathrm{e}-03 \quad 4.6137 \mathrm{e}-03$ $1248.4708 \mathrm{e}-03 \quad 9.6438 \mathrm{e}-05 \quad 2.8573 \mathrm{e}-05 \quad 1.0436 \mathrm{e}-03 \quad 6.9268 \mathrm{e}-03 \quad 2.9609 \mathrm{e}-03 \quad 4.5925 \mathrm{e}-03$ $1258.4232 \mathrm{e}-03 \quad 9.5672 \mathrm{e}-05 \quad 2.8231 \mathrm{e}-05 \quad 1.0308 \mathrm{e}-03 \quad 6.8879 \mathrm{e}-03 \quad 2.9618 \mathrm{e}-03 \quad 4.5722 \mathrm{e}-03$ $1268.3707 e-03 \quad 9.4889 e-05 \quad 2.7877 e-05 \quad 1.0182 e-03 \quad 6.8451 e-03 \quad 2.9623 e-03 \quad 4.5535 e-03$ iter continuity $\mathrm{x}$-velocity y-velocity z-velocity energy $\mathrm{k}$ omega $1278.3151 \mathrm{e}-03 \quad 9.4100 \mathrm{e}-05 \quad 2.7510 \mathrm{e}-05 \quad 1.0064 \mathrm{e}-03 \quad 6.7996 \mathrm{e}-03 \quad 2.9625 \mathrm{e}-03 \quad 4.5371 \mathrm{e}-03$ $1288.2584 \mathrm{e}-03 \quad 9.3282 \mathrm{e}-05 \quad 2.7142 \mathrm{e}-05 \quad 9.9512 \mathrm{e}-04 \quad 6.7532 \mathrm{e}-03 \quad 2.9622 \mathrm{e}-03 \quad 4.5228 \mathrm{e}-03$ $1298.2028 \mathrm{e}-03 \quad 9.2457 \mathrm{e}-05 \quad 2.6770 \mathrm{e}-05 \quad 9.8465 \mathrm{e}-04 \quad 6.7078 \mathrm{e}-03 \quad 2.9614 \mathrm{e}-03 \quad 4.5108 \mathrm{e}-03$ $1308.1486 \mathrm{e}-03 \quad 9.1621 \mathrm{e}-05 \quad 2.6402 \mathrm{e}-05 \quad 9.7487 \mathrm{e}-04 \quad 6.6634 \mathrm{e}-03 \quad 2.9601 \mathrm{e}-03 \quad 4.5006 \mathrm{e}-03$ $1318.0941 e-03 \quad 9.0793 e-05 \quad 2.6029 e-05 \quad 9.6588 e-04 \quad 6.6188 e-03 \quad 2.9583 e-03 \quad 4.4917 e-03$ $1328.0406 \mathrm{e}-03 \quad 8.9971 \mathrm{e}-05 \quad 2.5670 \mathrm{e}-05 \quad 9.5761 \mathrm{e}-04 \quad 6.5751 \mathrm{e}-03 \quad 2.9558 \mathrm{e}-03 \quad 4.4838 \mathrm{e}-03$
$0: 03: 53$ $0: 03: 50$ $0: 03: 47$ $0: 03: 44$ $0: 03: 40$ $0: 03: 37$ $0: 03: 34$ time/iter $0: 03: 31 \quad 67$ $0: 03: 28 \quad 66$ $0: 03: 25 \quad 65$ $0: 03: 22 \quad 64$ $0: 03: 18 \quad 63$ $0: 03: 15 \quad 62$ $0: 03: 12 \quad 61$ $0: 03: 0960$ $0: 03: 06 \quad 59$ $0: 03: 03 \quad 58$ 0:03:00 57 time/iter $0: 02: 57 \quad 56$ $0: 02: 53 \quad 55$ $0: 02: 50 \quad 54$ $0: 02: 47 \quad 53$ $0: 02: 44 \quad 52$ $0: 02: 41 \quad 51$ $0: 02: 38 \quad 50$ $0: 02: 3549$ $0: 02: 31 \quad 48$ $0: 02: 28 \quad 47$ $0: 02: 25 \quad 46$ time/iter $0: 02: 22 \quad 45$ $0: 02: 1944$ $0: 02: 16 \quad 43$ $0: 02: 13 \quad 42$ $0: 02: 0941$ $0: 02: 06 \quad 40$ $0: 02: 03 \quad 39$ $0: 02: 00 \quad 38$ $0: 01: 57 \quad 37$ $0: 01: 54 \quad 36$ $0: 01: 51 \quad 35$ time/iter $0: 01: 48 \quad 34$ $0: 01: 44 \quad 33$ $0: 01: 41 \quad 32$ $0: 01: 38 \quad 31$ $0: 01: 35 \quad 30$ $0: 01: 32 \quad 29$ $0: 01: 28 \quad 28$ $0: 01: 25 \quad 27$ $0: 01: 22 \quad 26$ $0: 01: 19 \quad 25$ $0: 01: 16 \quad 24$ time/iter 0:01:13 23 0:01:09 22 $0: 01: 06 \quad 21$ $0: 01: 03 \quad 20$ $0: 01: 00 \quad 19$ $0: 00: 57 \quad 18$ 
$\begin{array}{llllllllll}133 & 7.9872 e-03 & 8.9162 e-05 & 2.5319 e-05 & 9.4972 e-04 & 6.5315 e-03 & 2.9527 e-03 & 4.4767 e-03 & 0: 00: 54 & 17\end{array}$ $134 \quad 7.9342 e-03 \quad 8.8383 e-05 \quad 2.4981 e-05 \quad 9.4219 e-04 \quad 6.4881 e-03 \quad 2.9490 e-03 \quad 4.4696 e-03$ $1357.8796 \mathrm{e}-03 \quad 8.7624 \mathrm{e}-05 \quad 2.4645 \mathrm{e}-05 \quad 9.3491 \mathrm{e}-04 \quad 6.4435 \mathrm{e}-03 \quad 2.9447 \mathrm{e}-03 \quad 4.4619 \mathrm{e}-03$ $1367.8231 e-03 \quad 8.6926 e-05 \quad 2.4317 e-05 \quad 9.2756 e-04 \quad 6.3972 e-03 \quad 2.9397 e-03 \quad 4.4534 e-03$ $1377.7635 \mathrm{e}-03 \quad 8.6289 \mathrm{e}-05 \quad 2.3987 \mathrm{e}-05 \quad 9.2001 \mathrm{e}-04 \quad 6.3485 \mathrm{e}-03 \quad 2.9339 \mathrm{e}-03 \quad 4.4441 \mathrm{e}-03$ iter continuity $\mathrm{x}$-velocity $\mathrm{y}$-velocity $\mathrm{z}$-velocity $\mathrm{k}$ omega $1387.7025 \mathrm{e}-03 \quad 8.5714 \mathrm{e}-05 \quad 2.3665 \mathrm{e}-05 \quad 9.1241 \mathrm{e}-04 \quad 6.2986 \mathrm{e}-03 \quad 2.9275 \mathrm{e}-03 \quad 4.4339 \mathrm{e}-03$ $1397.6410 \mathrm{e}-03 \quad 8.5193 e-05 \quad 2.3350 \mathrm{e}-05 \quad 9.0457 \mathrm{e}-04 \quad 6.2483 \mathrm{e}-03 \quad 2.9204 \mathrm{e}-03 \quad 4.4227 \mathrm{e}-03$ $1407.5782 \mathrm{e}-03 \quad 8.4752 \mathrm{e}-05 \quad 2.3039 \mathrm{e}-05 \quad 8.9659 \mathrm{e}-04 \quad 6.1970 \mathrm{e}-03 \quad 2.9127 \mathrm{e}-03 \quad 4.4114 \mathrm{e}-03$ $1417.5143 e-03 \quad 8.4359 e-05 \quad 2.2739 e-05 \quad 8.8852 e-04 \quad 6.1448 e-03 \quad 2.9042 e-03 \quad 4.4001 e-03$ $1427.4512 \mathrm{e}-03 \quad 8.4048 \mathrm{e}-05 \quad 2.2436 \mathrm{e}-05 \quad 8.8034 \mathrm{e}-04 \quad 6.0931 \mathrm{e}-03 \quad 2.8952 \mathrm{e}-03 \quad 4.3887 \mathrm{e}-03$ $\begin{array}{lllllllll}143 & 7.3896 \mathrm{e}-03 & 8.3788 \mathrm{e}-05 & 2.2143 \mathrm{e}-05 & 8.7206 \mathrm{e}-04 & 6.0428 \mathrm{e}-03 & 2.8856 \mathrm{e}-03 & 4.3769 \mathrm{e}-03\end{array}$ $1447.3298 \mathrm{e}-03 \quad 8.3558 \mathrm{e}-05 \quad 2.1847 \mathrm{e}-05 \quad 8.6362 \mathrm{e}-04 \quad 5.9939 \mathrm{e}-03 \quad 2.8754 \mathrm{e}-03 \quad 4.3647 \mathrm{e}-03$ $1457.2726 \mathrm{e}-03 \quad 8.3374 \mathrm{e}-05 \quad 2.1568 \mathrm{e}-05 \quad 8.5509 \mathrm{e}-04 \quad 5.9472 \mathrm{e}-03 \quad 2.8648 \mathrm{e}-03 \quad 4.3523 \mathrm{e}-03$ $1467.2200 \mathrm{e}-03 \quad 8.3279 \mathrm{e}-05 \quad 2.1290 \mathrm{e}-05 \quad 8.4656 \mathrm{e}-04 \quad 5.9042 \mathrm{e}-03 \quad 2.8538 \mathrm{e}-03 \quad 4.3402 \mathrm{e}-03$ $147 \quad 7.1724 \mathrm{e}-03 \quad 8.3227 \mathrm{e}-05 \quad 2.1019 \mathrm{e}-05 \quad 8.3802 \mathrm{e}-04 \quad 5.8653 \mathrm{e}-03 \quad 2.8424 \mathrm{e}-03 \quad 4.3281 \mathrm{e}-03$ $1487.1307 \mathrm{e}-03 \quad 8.3209 \mathrm{e}-05 \quad 2.0759 \mathrm{e}-05 \quad 8.2950 \mathrm{e}-04 \quad 5.8312 \mathrm{e}-03 \quad 2.8307 \mathrm{e}-03 \quad 4.3159 \mathrm{e}-03$ iter continuity $\mathrm{x}$-velocity y-velocity $\mathrm{z}$-velocity energy $\mathrm{k}$ omega $1497.0975 e-03 \quad 8.3242 e-05 \quad 2.0507 e-05 \quad 8.2107 e-04 \quad 5.8041 e-03 \quad 2.8187 e-03 \quad 4.3042 e-03$ $1507.0739 e-03 \quad 8.3330 e-05 \quad 2.0260 e-05 \quad 8.1260 e-04 \quad 5.7849 e-03 \quad 2.8066 e-03 \quad 4.2937 e-03$ $0: 00: 51 \quad 16$ $0: 00: 47 \quad 15$ $0: 00: 44 \quad 14$ $0: 00: 41 \quad 13$ time/iter $0: 00: 38 \quad 12$ $0: 00: 3511$ $0: 00: 32 \quad 10$ $0: 00: 28 \quad 9$ $0: 00: 25 \quad 8$ $0: 00: 22 \quad 7$ $0: 00: 19 \quad 6$ $0: 00: 16 \quad 5$ $0: 00: 13 \quad 4$ $0: 00: 093$ 0:00:06 2 time/iter 0:00:03 1 $0: 00: 00$

$>$ /define/models/unsteady-2nd-order yes

>/solve/monitors/surface/set-monitor x-velocity-turbineinlet "Area-Weighted Average" x-velocity turbine () no no yes "ave_x_velocity_-turbineinlet_area_weighted" 1 yes flow

>/solve/monitors/surface/set-monitor abl-velocity-turbineinlet "Area-Weighted Average" abl turbine () no no yes "ave_abl_velocity_turbineinlet_area_weighted" 1 yes flow

$>$ /solve/monitors/surface/set-monitor mass-flow-rate-turbineinlet "Mass Flow Rate" turbine () no no yes "mass_flow_rate_turbineinlet" 1 yes flow

> /solve/monitors/surface/set-monitor augmented-power "Integral" power_aug turbine () no no yes "power_aug" 1 yes flow

>/solve/monitors/surface/set-monitor non-augmented-power "Integral" power_abl turbine () no no yes "power_abl" 1 yes flow

$>$ /file/auto-save data-frequency 200

$>$ /solve/set time-step 1

> /solve/dual-time-iterate 70020

Updating solution at time levels $\mathrm{N}$ and $\mathrm{N}-1$.

done.

iter continuity $\mathrm{x}$-velocity $\mathrm{y}$-velocity $\mathrm{z}$-velocity energy $\mathrm{k}$ omega time/iter $1507.0739 e-03 \quad 8.3330 e-05 \quad 2.0260 e-05 \quad 8.1260 e-04 \quad 5.7849 e-03 \quad 2.8066 e-03 \quad 4.2937 e-03 \quad 0: 01: 03 \quad 20$ $\begin{array}{llllllllll}151 & 7.0568 e-03 & 8.3438 e-05 & 2.0026 e-05 & 8.0396 e-04 & 5.7709 e-03 & 2.7695 e-03 & 4.2933 e-03 & 0: 01: 01 & 19\end{array}$ $\begin{array}{lllllllllll}152 & 7.0542 e-03 & 8.3533 e-05 & 1.9747 e-05 & 7.9212 e-04 & 5.7688 e-03 & 2.6403 e-03 & 4.2828 e-03 & 0: 00: 58 & 18\end{array}$

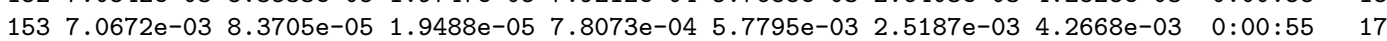
$\begin{array}{llllllllll}154 & 7.0953 e-03 & 8.3841 e-05 & 1.9212 e-05 & 7.6918 e-04 & 5.8025 e-03 & 2.4043 e-03 & 4.2472 e-03 & 0: 00: 51 & 16\end{array}$ $\begin{array}{llllllllll}155 & 7.1350 \mathrm{e}-03 & 8.4130 \mathrm{e}-05 & 1.8980 \mathrm{e}-05 & 7.5750 \mathrm{e}-04 & 5.8350 \mathrm{e}-03 & 2.2966 \mathrm{e}-03 & 4.2253 \mathrm{e}-03 & 0: 00: 48 & 15\end{array}$ $\begin{array}{llllllllll}156 & 7.1824 \mathrm{e}-03 & 8.4258 \mathrm{e}-05 & 1.8782 \mathrm{e}-05 & 7.4588 \mathrm{e}-04 & 5.8737 \mathrm{e}-03 & 2.1955 \mathrm{e}-03 & 4.2014 \mathrm{e}-03 & 0: 00: 45 & 14\end{array}$ $\begin{array}{llllllllll}157 & 7.2340 e-03 & 8.4269 e-05 & 1.8581 e-05 & 7.3362 e-04 & 5.9159 e-03 & 2.1004 e-03 & 4.1752 e-03 & 0: 00: 42 & 13\end{array}$ $\begin{array}{llllllllll}158 & 7.2874 \mathrm{e}-03 & 8.4255 \mathrm{e}-05 & 1.8411 \mathrm{e}-05 & 7.2231 \mathrm{e}-04 & 5.9595 \mathrm{e}-03 & 2.0111 \mathrm{e}-03 & 4.1480 \mathrm{e}-03 & 0: 00: 38 & 12\end{array}$ $1597.3403 e-03 \quad 8.4283 e-05 \quad 1.8257 e-05 \quad 7.1084 e-04 \quad 6.0028 e-03 \quad 1.9272 e-03 \quad 4.1192 e-03 \quad 0: 00: 35 \quad 11$ $160 \quad 7.3897 e-03 \quad 8.4314 e-05 \quad 1.8142 e-05 \quad 7.0042 e-04 \quad 6.0431 e-03 \quad 1.8484 e-03 \quad 4.0889 e-03 \quad 0: 00: 32 \quad 10$ iter continuity $\mathrm{x}$-velocity y-velocity z-velocity energy $\mathrm{k}$ omega time/iter $\begin{array}{llllllllll}161 & 7.4349 \mathrm{e}-03 & 8.4226 \mathrm{e}-05 & 1.8016 \mathrm{e}-05 & 6.9048 \mathrm{e}-04 & 6.0801 \mathrm{e}-03 & 1.7744 \mathrm{e}-03 & 4.0576 \mathrm{e}-03 & 0: 00: 29 & 9\end{array}$ $1627.4731 e-03 \quad 8.3890 e-05 \quad 1.7917 e-05 \quad 6.7957 e-04 \quad 6.1113 e-03 \quad 1.7049 e-03 \quad 4.0252 e-03 \quad 0: 00: 26 \quad 8$ $\begin{array}{llllllllll}163 & 7.5050 e-03 & 8.3535 e-05 & 1.7823 e-05 & 6.6997 e-04 & 6.1373 e-03 & 1.6396 e-03 & 3.9929 e-03 & 0: 00: 22 & 7\end{array}$ 
$1647.5332 \mathrm{e}-03 \quad 8.3193 \mathrm{e}-05 \quad 1.7710 \mathrm{e}-05 \quad 6.6144 \mathrm{e}-04 \quad 6.1603 \mathrm{e}-03 \quad 1.5782 \mathrm{e}-03 \quad 3.9614 \mathrm{e}-03 \quad 0: 00: 19$

$1657.5546 \mathrm{e}-03 \quad 8.2816 \mathrm{e}-05 \quad 1.7611 \mathrm{e}-05 \quad 6.5253 \mathrm{e}-04 \quad 6.1778 \mathrm{e}-03 \quad 1.5205 \mathrm{e}-03 \quad 3.9304 \mathrm{e}-03 \quad 0: 00: 16$

$1667.5693 \mathrm{e}-03 \quad 8.2507 \mathrm{e}-05 \quad 1.7486 \mathrm{e}-05 \quad 6.4471 \mathrm{e}-04 \quad 6.1898 \mathrm{e}-03 \quad 1.4662 \mathrm{e}-03 \quad 3.8990 \mathrm{e}-03 \quad 0: 00: 13$

$167 \quad 7.5804 \mathrm{e}-03 \quad 8.1951 \mathrm{e}-05 \quad 1.7344 \mathrm{e}-05 \quad 6.3682 \mathrm{e}-04 \quad 6.1988 \mathrm{e}-03 \quad 1.4150 \mathrm{e}-03 \quad 3.8676 \mathrm{e}-03 \quad 0: 00: 10$

$1687.5880 \mathrm{e}-03 \quad 8.1359 \mathrm{e}-05 \quad 1.7202 \mathrm{e}-05 \quad 6.2941 \mathrm{e}-04 \quad 6.2050 \mathrm{e}-03 \quad 1.3668 \mathrm{e}-03 \quad 3.8362 \mathrm{e}-03 \quad 0: 00: 06$

$1697.5949 \mathrm{e}-03 \quad 8.0747 \mathrm{e}-05 \quad 1.7049 \mathrm{e}-05 \quad 6.2325 \mathrm{e}-04 \quad 6.2107 \mathrm{e}-03 \quad 1.3213 \mathrm{e}-03 \quad 3.8031 \mathrm{e}-03 \quad 0: 00: 03$

$1707.6023 e-03 \quad 8.0073 e-05 \quad 1.6868 \mathrm{e}-05 \quad 6.1726 \mathrm{e}-04 \quad 6.2167 \mathrm{e}-03 \quad 1.2785 \mathrm{e}-03 \quad 3.7684 \mathrm{e}-03 \quad 0: 00: 00$

Flow time $=1 \mathrm{~s}$, time step $=1$

699 more time steps

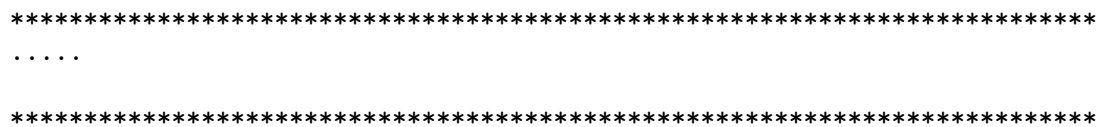

Updating solution at time levels $\mathrm{N}$ and $\mathrm{N}-1$.

done.

iter continuity $\mathrm{x}$-velocity $\mathrm{y}$-velocity $\mathrm{z}$-velocity energy omega time/iter

! 2985 solution is converged

$29857.7169 e-04 \quad 5.5841 e-06 \quad 6.4118 e-07 \quad 1.3866 e-04 \quad 6.3103 e-04 \quad 7.5365 e-04 \quad 5.5183 e-04 \quad 0: 01: 14 \quad 20$

! 2986 solution is converged

$29867.7994 e-04 \quad 5.8968 e-06 \quad 6.5650 e-07 \quad 1.3966 e-04 \quad 6.3777 e-04 \quad 7.5339 e-04 \quad 5.5250 e-04 \quad 0: 01: 10 \quad 19$

Flow time $=699 \mathrm{~s}$, time step $=699$

1 more time step

Updating solution at time levels $\mathrm{N}$ and $\mathrm{N}-1$.

done.

iter continuity $\mathrm{x}$-velocity $\mathrm{y}$-velocity $\mathrm{z}$-velocity $\mathrm{k}$ omega time/iter

! 2986 solution is converged

$29867.7994 e-04 \quad 5.8968 e-06 \quad 6.5650 e-07 \quad 1.3966 e-04 \quad 6.3777 e-04 \quad 7.5339 e-04 \quad 5.5250 e-04 \quad 0: 01: 14 \quad 20$

! 2987 solution is converged

$2987 \quad 7.9066 e-04 \quad 6.0848 e-06 \quad 6.5593 e-07 \quad 1.4012 e-04 \quad 6.4654 e-04 \quad 7.5317 e-04 \quad 5.5275 e-04 \quad 0: 01: 10 \quad 19$

Flow time $=700 \mathrm{~s}$, time step $=700$

$>$ /file/write-case Rt_1_Rx_0_Ry_0_A0A_0.cas.gz

/file/write-data Rt_1_Rx_0_Ry_0_AOA_0.dat.gz

/report/summary yes "report.sum"

/exit

$* * * * * * * * * * * * * * * * * * * * * * * * * * * * * * * * * * * * * * * * * * * * * * * * * * * * * * * * * * * * * * * * * * * * * * * * * * *$

$\begin{array}{ll}\text { Number cpus } & =8 \\ \text { Queue } & =\mathrm{mpi} \\ \text { Jobid } & =78537 . \text { gup-hn.guppy.sharcnet } \\ \text { Version } & =3 \mathrm{ddp} \\ \text { Journal file } & =\text { test.jou } \\ \text { Date job started } & =\text { Thu Mar } 31 \text { 21:22:46 EDT } 2011 \\ \text { Date job finished } & =\text { Fri Apr } 1 \text { 19:11:57 EDT } 2011\end{array}$




\section{Bibliography}

[1] Bruce Wind Turbine. Ontario Power Generation Inc., 2001.

[2] ANSYS FLUENT-Solver Theory. ANSYS Inc., 2009.

[3] GISS Surface, Temperature Analysis, volume 2. Goddard Institute for Space Studies,National Aeronautics and Space Administration, 2011.

[4] Wind and weater statistic Toronto Island. Windfinder.com, 2011.

[5] M. J. Blanch A. G. Duuton, J. A. Halliday. The Feasibility of Building-Mounted/Integrated Wind Turbines (BUWTs):Achieving their potential for carbon emission reductions. Energy Research Unit, CCLRC, 2005.

[6] L. Bryans N. Jenkins D. Milborrow M. O’Malley R. Watson B. Fox, D. Flynn and O. Anaya-Lara. Wind Power Integration:Connection and system operational aspects. The Institute of Engineering and Technology, 2007.

[7] P.W. Bearman and A.J. Wadcock. The interaction between a pair of circular cylinders normal to a stream. Fluid Mechanics, 61, 499., 1973.

[8] L. Chen and J. Tu. A Large-Eddy Simulation of Two Side-by-Side Circular Cylinders in Cross Flow. Maritime Platforms Devision, DSTO Melbourne and School of Mechanical and Manufacturing Engineering, RMIT University, Autralia.

[9] Robert K. W. Dannecker and Andrew D. Grant. Integrated Ducted Wind Turbine Module. University of Strathclyde, Glasgow, UK, 2002.

[10] L.G. Margolin F.F. Grinstein and W.J. Rider. Implicit Large Eddy Simulation. 2007.

[11] M. Gaster. Vortex shedding from slender cones at low Reynolds number. Journal of Fluid Mechanics, 38, 565-576, 1969.

[12] M. Gaster. Vortex shedding from circular cylinders at low Reynolds number. Journal of Fluid Mechanics, 46, 746-756, 1971. 
[13] Gorlov Alexander M. Silantyv Valentin M. Gorban, Alexander N. Limits of the Turbine Efficiency for Free Fluid Flow. Journal of Energy Resource Technology, ASME, Vol. 123, pg311-317 December, 2001.

[14] H. Hamm. Optimization of Wind Augmenters for Urban Power Generation. Ryerson University, 2006.

[15] J. D. Holmes. Wind Loading of Structures. Spoon Press, New York, NY, 2001.

[16] X. Jiang and C.H. Lai. Numerical Techniques for Direct and Large Eddy Simulation. 2009.

[17] H.J. Kim and P.A. Durbin. Investigation of the flow between a pair of circular cylinders in the floppy regime. Lewis Research Center, Cleveland, OHm USA, 1988.

[18] C. W. Rowley M. A. Green and G. Haller. Detection of Langrangian coherent structures in threedimentional turbulence. Cambridge University Press, 2007.

[19] S. Mertens. Wind Energy in the Built Environment. Multi-Science, United Kingdom, 2006.

[20] United Nations. Kyoto Protocol to the United Nations Framework Convention on Climate Change. 1998.

[21] P. Piccirillo and C.W. Van Atta. An experimental study of vortex shedding behind linearly tapered cylinders at low Reynolds number. Journal of Fluid Mechanics, 246, 163-195, 1993.

[22] A. Harries S. Stankovic, N. Campbell. Urban Wind Energy. Sterling, VA: Earthscan, 2009.

[23] Ian J. Sobey. Oscillatory Flows at Intermediate Strouhal Number in Asymmerty Channels. Journal of Fluid Mechanics, 125, pg359-373, 1982.

[24] M. M. Zdravkovich. Flow around Circular Cylinders; Volume 1. Fundamentals. Oxford Science Publications, 1997. 
